\title{
AC TRANSMISSION LINE FIELD MEASUREMENTS
}

WORK PERFORMED UNDER CONTRACT

EA-77-A-01-6010-A0-18-1

PUBLICATION DATE: NOVEMBER 1977

NATIONAL BUREAU OF STANDARDS

PREPARED FOR

U.S. DEPARTMENT OF ENERGY

DIVISION OF ELECTRIC ENERGY SYSTEMS 


\section{DISCLAIMER}

This report was prepared as an account of work sponsored by an agency of the United States Government. Neither the United States Government nor any agency Thereof, nor any of their employees, makes any warranty, express or implied, or assumes any legal liability or responsibility for the accuracy, completeness, or usefulness of any information, apparatus, product, or process disclosed, or represents that its use would not infringe privately owned rights. Reference herein to any specific commercial product, process, or service by trade name, trademark, manufacturer, or otherwise does not necessarily constitute or imply its endorsement, recommendation, or favoring by the United States Government or any agency thereof. The views and opinions of authors expressed herein do not necessarily state or reflect those of the United States Government or any agency thereof. 


\section{DISCLAIMER}

Portions of this document may be illegible in electronic image products. Images are produced from the best available original document. 
NOTICE

This publication was prepared as an account of work sponsored by an agency of the United States Government. Neither the United States Government nor any agency thereof, nor any of their employees, makes any warranty, express or implicd, or assumes any legal liability or responsibility for any third party's use or the results of such use of any information, apparatus, products or process disclosed in this document, or represents that its use by such a third party would not infringe privately owned rights.

Available from:

National Technical Information Service (NTIS)

U.S. Department of Commerce

5285 Port Royal Road

Springfield, Virginia 22161

Price: Printed copy: $\$ 5,00$

Microfiche: $\quad \$ 3.00$ 


\title{
AC TRANSMISSION LINE FIELD MEASUREMENTS
}

This report was propared as an arrsount of work sponsored by the United States Govemment. Neither the United States nor the United States Department of Energy, nor any of their employees, nor any of their contractors, subcontractors, or their employees. makes any warranty, express or implied, or assumes any legal liability of responsibility for the accuracy, completeness or usefulness of any information apparatus, product or process disclosed, or represents that its use would not infringe privately owned rights.

\section{F. RALPH KUTIER}

MARTIN MISAKIAN

INSTITUTE FOR BASIC STANDARDS

NATIONAL BUREAU OF STANDARDS $950 \quad 3368$ WASHINGTON, D.C. 20234

PUBLICATION DATE: NOVEMBER 1977

PREPARED FOR THE

\section{U.S. DEPARTMENT OF ENERGY}

\author{
ASSISTANT SECRETARY FOR ENERGY TECHNOLOGY \\ DIVISION OF ELECTRIC ENERGY SYSTEMIS \\ WASHINGTON, D.C., 20545
}

WORK PERFORMED UNDER CONTRACT NO.

EA-77-A-01-6010-AO 18-1 
AC TRANSMISSION LINE FIELD MEASUREMENTS

Contract No. EA-77-A-01-6010-A0 18-1

NBS PROJECT No. 2110416

Prepared for

Department of Energy

Electric Energy Systems Division

Washington, D.C. 20545

Prepared by

F. Ralph Kotter

Martin Misakian

Institute for Basic St.andards

National Bureau of Standards

Washington, D.C. 20234

September 30, 1977 


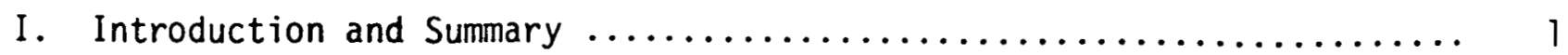

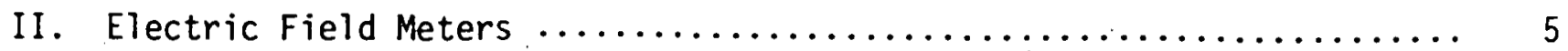

A. Instrumentation and Theory of Operation $\ldots \ldots \ldots \ldots \ldots \ldots \ldots . . \ldots \ldots$

B. Parameters Influencing Instrument Performance $\ldots \ldots \ldots \ldots \ldots \ldots . \ldots$

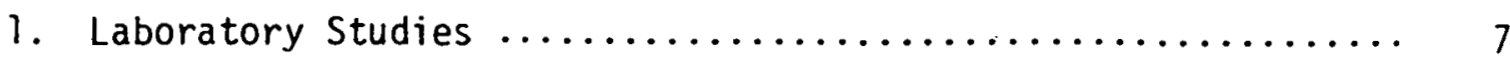

(a) Temperature and Humidity Effects ................ 7

(b) The Effect of Electrical Leakage Along the Handle ...... 8

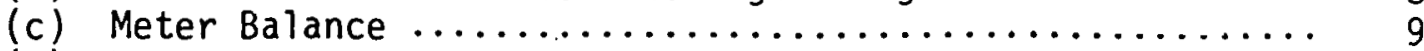

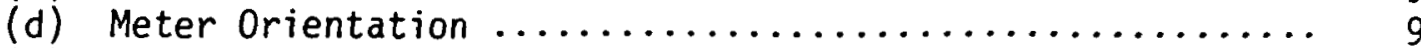

(e) The Influence of Ambient Magnetic Field ............. 10

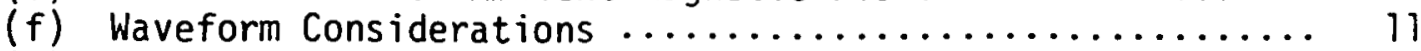

(g) Measurement of Non-uniform E-fields ............. 13

2. Field Studies .............................. 17

(a) Effect of Proximity of the Observer .............. 17

(b) Comparison of U.S. and Soviet E-field ............. ig Measurement Procedures

C. Calibration of Electric Field Meters .................... 23

1. Introduction ............................ 23

2. Finite Size of Plates and Fringing Fields ............. 24

3. Modification of Fringing Fields, Compensating ........... 25

Procedures and Generation of a Calibration Field

4. Parallel-Plate Calibration Structures ................. 34

5. Current Injection Calibration ................... 35

III. Magnetic Field Measurements ........................... 40

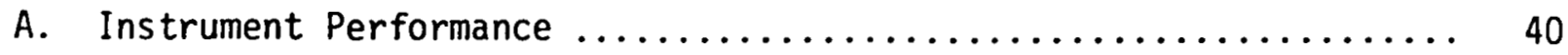

B. Calibration of Magnetic Field Meters .................... 41

Appendix A. Current Induced in Conducting Sphere by Quasistatic ....... 46 Electric Field From Point Charge

Appendix B. The Electric Field Between Parallel Plates--One ........... 49 Plate Grounded

(i) Perturbation of Surfare. Charge Density By ........... 49 Field Meter

(ii) Perturbation of Surface Charge Density By ............ 57 Nearby Ground Planes

(iii) Effectiveness of Guard Rings For Reducing ............ 62 External Perturbations

(iv) Parallel Plates with Guard Rings ................. 65

Appendix C. Ground Referenced Electric Field Meters ............... 71

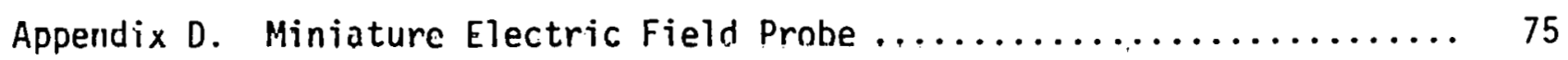

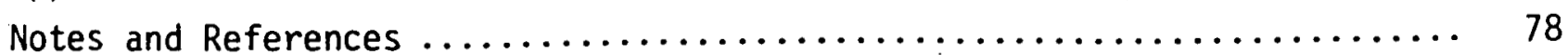




\section{Introduction and Summary}

The concern in recent years over the environmental effects of electric and magnetic fields from high voltage transmission lines has also focused attention on the accuracy of measurements of these fields. This research effort has had several objectives: (1) to evaluate the instrumentation available for measuring the near fields of ac transmission lines; (2) to evaluate and suggest criteria for calibration; (3) to evaluate field-use procedures; (4) to establish a calibration service at NBS for these instruments; and (5) to document the results of this study in a form useful as a guide for all concerned with such measurements .

A portion of the material presented in this report has been contributed to an IEEE Working Group Report, "Measurement of Electric and Magnetic Fields From Alternating Current Power Lines," presented at the 1977. Summer Power Meeting.

The instrumentation study has been limited primarily to two ac electric field (E-field) meters commercially available in the United States. One of these has a magnetic field probe accessory which has also been evaluated. *

Laboratory studies (II.B.1) of parameters influencing the performance of a small sample of E-field meters are summarized in Table $I$.

*This unit is also designed to measure space pntential, induced short circuit current, and induced open circuit voltage. These functions were not evaluated. 
Table I

\section{Performance Evaluation of Several}

E-Field Meters

\section{Parameter}

Type A

Field Meter
Type B

Field Meter

\section{Temperature}

(temperature

coefficients)

Relative Humidity

(change from 20 to $95 \%$ )

Handle Leakage

Frequency

Waveform

Ambient Magnetic

Field
$0.1-0.2 \% /{ }^{\circ} \mathrm{C}(\mathrm{a})$

$$
<.1 \%(c)
$$

Not likely to be
source of error $(d)$

Independent of

frequency over

the power fre-

quency range

Sensilive to harmonic distortion. Maximum error is less than $1 / 3$ the $\%$ distortion.

Negligible influence
$0.1-0.2 \% /{ }^{\circ} \mathrm{C}(\mathrm{a})$
$0.4 \%{ }^{\circ} \mathrm{C}(\mathrm{b})$

$$
\begin{aligned}
& <1 \%(a) \\
& 23 \%(b)
\end{aligned}
$$

A potential source of error

Indication is a function of trequency

Quite sensitive to harmonic distortion. Maximum error can be approximately the same as the \% distortiun.

Negligible influence

\footnotetext{
(a) two instruments tested

(b) one instrument tested gave these values--indicating a possibly defective unit

(c) one of the two instruments tested initially exhibited a much larger effect but this disappeared with subsequent use

(d) surface contamination of the yoke is a potential source of error under conditions of high humidity
} 
Yet another parameter, the observer's proximity, has also been studied in the field under EHV lines. It has been found that in making measurements at a height of one meter above the ground, a standing observer can perturb (reduce) the field measurement by $5 \%$ when 1.4 to $2.1 \mathrm{~m}$ away from the field meter (Section II.B.2.a).

The proximity effects of the observer when U.S. field meters with short handles are used to measure E-fields at a height of $1.8 \mathrm{~m}$ (attempted simulation of reported Soviet practice, circa 1972) are highly variable (Section II.B.2.b). Simulation results were found to be dependent on such factors as the orientation of the observer's hand and arm relative to the meter, probe (sensing electrode) geometry, the angle of meter handle with respect to the vertical, and location relative to transmission lines. The proximity effects produced significant enhancements in the field measurement (>20\%) for all configurations examined.

Experimental verification is provided of theoretical studies which show that the field meters, although designed for use in uniform fields, can under certain conditions be used to within an accuracy of approximately $7 \%$ in non-uniform fields which vary as much as $30 \%$ over the major dimension of the meter (Section II.B.I.g).

Details of extensive model and full-scale studies of parallel-plate electrode structures for the calibration of E-field meters are presented (Section II.C and Appendix B). The investigations consider the effects of guard rings, guard bands, method of energizing the plates, nearby ground planes (walls, floor), as well as the field meter itself on the field between the plates. These studies have led to the conclusion that $1.5 \times 1.5$ meter plates separated by 0.75 meter without guard rings or $1 \times 1$ meter plates separated by 0.75 meter and equipped with four properly voltage-graded guard rings can be satisfactory for calibration of the field meters studied with an accuracy of better than $2 \%$. 
Calibrations of ac field meters to an accuracy of $1 \%$ are now available from the National Bureau of Standards.

The relative merits of several current-injection "calibration" circuits are explored (Section II.C.5). Different circuits are recommended for use with the two field meters studied and precautions in their use outlined which, if followed, make this a valuable and convenient technique for frequent calibration checks.

Results describing the performance of the magnctic ficld probe referred to above are presented, as well as an analysis of the accuracy to be expected from the use of simple multiturn rectangular loops for the calibration of such meters (Section III). 


\section{Electric Field Meters}

A. Instrumentation and Theory of Operation

The two commercially available electric field meters currently available in the U.S. are of the so called "free body" type. That is, both are self-contained units with sensing electrodes which are integral with the battery-operated circuits and read-out devices; the meters are introduced into the electric field (E-field) to be measured at the end of an insulating rod and have no connection to ground except possibly via the observer holding the handle. Sketches illustrating their approximate geometry are shown in Figure la; the side dimensions range from . $7 \mathrm{~cm}$ to $\approx 20 \mathrm{~cm}$. It. shnuld be noted that ground referenced meters have also been devised $[1,2]$ but that they can be used only under special conditions described in Appendix $C$.

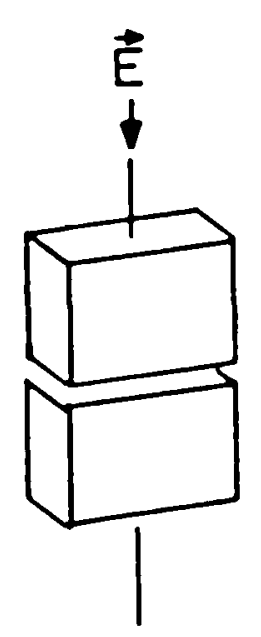

(a) Geometries of commercial U.S. E-field meters.

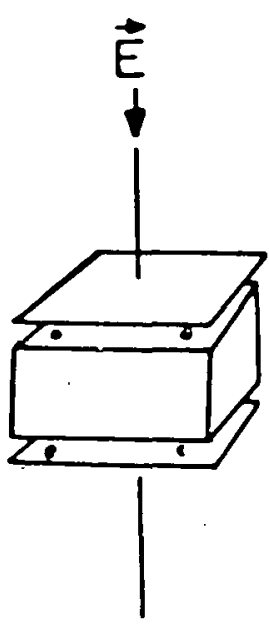

(b) Spherical E-field probc.

Figure 1.

Briefly the theory of operation of free-body meters can be understood by considering an uncharged conducting free body with two halves introduced into a uniform field $\bar{E}_{0}$. The charge induced on one of the halves is 


$$
Q=\int_{S / 2} \vec{D} \cdot d \vec{A}
$$

where $\vec{D}$. is the electric displacement and $\overrightarrow{d A}$ is an area element on half of the body with total surface area $S$. The case of a sphere divided into hemispheres by a plane normal to the electric field (Figure lb) yields the result that. [3]

$$
Q=3 \pi a^{2} \cdot \varepsilon_{0} E_{0}
$$

where $a$ is the radius of the sphere and $\varepsilon_{0}$ is the permittivity of free space. For less symmetric geometries (Figure la), the resull can be expressed as

$$
Q=k E_{0} E_{0}
$$

where $k$ is a constant dependent on geometry. If the electric field has a sinusoidal dependence, e.g., $E_{0}$ sin wt, the charge, given by Eq. 3, oscillates between the two halves and the current is

$$
I=\frac{d Q}{d t}=k \omega \varepsilon_{0} E_{0} \cos \omega t .
$$

It should be noted that the uniform E-field direction serves as an alignment axis for the field probe. The constant $k$ can be thought of as a field meter constant and is determined by calibrution.

One of the commercial E-field meters, designated Type $A$, is designed to determine $E_{0}$ by measuring a quantity proportional to the charges induced on the sensing electrodes; the other, designated Type $B$, is designed to determine $E_{0}$ by measuring a quantity proportional to the current between the electrodes. In both types the analog meter, while calibrated to read the rms value of a sinusoidal signal, actually responds to the average value of the rectified signal. Thus the 
presence of harmonics in the electric field being measured introduces an error which depends on the phase relationships of the harmonic components to the fundamental as well as on their magnitudes. Furthermore, the signal processing techniques used in the two instruments differ in their sensitivities to the harmonic components in the field waveform. The significance of these differences is discussed in Section II.B.I.f.

\section{B. Parameters Influencing. Instrument Performance.}

1. Laboratory Studies

(a) Temperature and Humidity Effects

Studies have been made of the influence of temperature and relative humidity on the analog output of the ac fieid measuring instruments. In addition lu lhe two instruments purchased for this study, three others, obtained on loan, were studied.

The behavior of three (one of Type $A$ and two of Type $B$ ) of the instruments was very similar--with a calibration midway in the temperature range between 0 and $40 .{ }^{\circ} \mathrm{C}$ ( 32 and $110^{\circ} \mathrm{F}$ ), each could be used over that complete range with a maximum uncertainty due to temperature of less than $\pm 5 \%$. (Temperature coefficients were of the order of 1 or 2 parts in $10^{3} /{ }^{\circ} \mathrm{C}$ ). The influence of relative humidity over the range from about $20 \%$ to $95 \%$ was less than $1 \%$. The results obtained with the fourth meter (Type B) were somewhat erratic; however, the influence of both temperature and relative humidity was significantly greater for this instrument than for the others. The meter indication at $40{ }^{\circ} \mathrm{C}$ differed from that at $0{ }^{\circ} \mathrm{C}$ by about $17 \%$ (a temperature coefficient in excess of $0.4 \% /{ }^{\circ} \mathrm{C}$ ) and, while the scatter in the data was large, the indication was that a change in relative humidity at constant temperature from 50 to $95 \%$ changed the meter indication about $3 \%$. The fifth meter (Type A) initially exhibited an extreme sensitivity to high relative humidity; however, this behavior was relatively short lived and 
the subsequent behavior was essentially identical with that of the first three mentioned above.

(b) The Effect of Electrical Leakage Along the Handle

The effect of electrical leakage along the supporting handles has been studied in a semi-quantitative way by introducing multi-megohm resistors of known magnitude in a conducting path along the handle to ground. The interpretation of the results is not exactiy straightforward since the effect of the resistive leakage has not been rigorously separated from the contribution of the stray capacitances; however, the results are believed adequate for the present purpose.

The two instruments which are currently commercially available differ in a significant way with respect to susceptibility to error from a leakage path to ground along the handle. In the Type B meter the section of the meter case to which the handle is connected is an active member of the field-sensing arrangement. In the Type A meter the handle is connected directly to the meter case, but both sensing elements are isolated to some extent from it.

Electrical leakage along the handle is not a source of error in Type A meters. In fact, in normal use the meter case can be tied directly to ground without introducing more than a few percent error. However, under high humidity conditions surface contamination of the yoke which connects the handle to the meter can introduce significant error as a result of the field distortion produced.

For the Type B meter, electrical leakage along the handle can be a significant source of error. It appears that. an effertive leakage resistance to ground of the order of $10^{10}$ ohms introduces an error of several percent. Measurements in an environmental chamber of the resistance between contacts placed about 1 meter apart on one handle gave a value of about $10^{10}$ ohms when the relative humidity was about $90 \%$. This result should be accepted only as an indication of the possible existence of a problem since the surface conductivity of an insulating 
material is usually strongly voltage dependent and, of course, greatly influenced by contamination of the surface resulting from even only ordinary handiing.

As has been pointed out by the designer of this meter, the effect of electrical leakage along the handle is not the same with the handle connected to the upper electrode as with it connected to the lower electrode. Thus in use, if the meter movement is well balanced and its reading is the same when it is inverted as when in the normal position, it may be assumed that electrical leakage to ground along the handle is introducing negligible error. However, this test can be applied only if the handle length is sufficient to eliminate the perturhing effert. of the observer in both orientations of the meter. In general, the perturbation can be expected to be somewhat larger with the meter in the inverted position (see Section II.B.1.d).

(c) Meter Balance

The question of mechanical balance of the meter movement is important. If a meter is not sufficiently well balanced it must be used in the same orientation with respect to the vertical as existed when it was calibrated. Of the five instruments so far tested, four were quite satisfactorily balanced,but the zero position of the fifth changed by as much as a full division as the orientation with respect to the vertical was varied.

\section{(d) Meter Orientation}

With field meters having geometrically symmetric sensing electrodes, a maximum reading is obtained when the meter is so oriented that the field is aligned with the meter axis--defined as the line passing through the centers of the two electrodes. The meter reading is then expected to follow a cosine law with respect to the angle between the field and the meter axis. However, the mounting of the indicating meter on the upper electrode and the handle-mounting bracket on the lower electrode of the Type B field meter introduce a significant 
asymmetry. The maximum reading is obtained with the top of the front surface of the Type $B$ meter tipped toward the observer about 15 degrees with respect to the field vector. The reading in this position is about $4 \%$ greater than when the meter is aligned with the field. If the vertical geometric axis of the field meter coincides with the field vector both during calibration and in use, this anomaly can be ignored except as it imposes a requirement for somewhat greater care in maintaining correct orientation during both calibration and use. An error will be introduced in attempting to use this meter to measure the vertical component of the field if a significant horizontal component in the direction of the handle exists. For this reason it appears that measurements with it in the vicinity of transmission lines should be made with the handle of the field meter parallel to the line. This same orientation should be used with any field meter when measurements of the maximum E-field are being sought because there are no field components parallel to the transmission line direction. Exceptions to this are locations near towers and other field perturbing objects.

(e) The Influence of Ambient Magnelic Field

Using a set of Helmholtz coils, a $60 \mathrm{~Hz}$ magnetic field of $1.2 \times 10^{-5}$ tesla $(0.120$ gauss $)$ was impressed on one of the instruments when set as for use in measuring an electric field. The test was repeated with several scale readings and for several orientations of the meter relative to the magnetic field vector. Similar tests were made with each of the five instruments referred to above. No significant influence of the magnetic field at this level was detected with any of the meters.

Tests using one field meter of Type $A$ and one of Type $B$ were repeated at field values near $1.1 \times 10^{-4}$ tesla ( 1.1 gauss). This is well in excess of values likely to be encountered near transmission lines. Negligible effects were observed witn a Type $A$ meter on the most sensitive E-field range (5 kV/m full scale). 
Perturbations of a few percent on the $1 \times 100 \mathrm{~V} / \mathrm{m}$ scale of the Type $B$ meter were evident, but negligible effects on the higher ranges were observed. The orientation of the meters with respect to the B-field direction was varied during each of the tests without significant effect.

\section{(f) Waveform Considerations}

While both Type A and Type B field meters are calibrated to read the rms value of a $60 \mathrm{~Hz}$ sinusoidal waveform, the analog meters actually respond to the average value of the rectified signal. The presence of harmonic components in the electric field waveform introduces errors which depend on both the magnitudes of the harmonic components and their phase relationships to the fundamental. Figure 2 shows the calculated measurement errors which are introduced when selected harmonic components are present. The calculation assumes that the harmonics are in phase with the fundamental, a condition which leads to maximum measurement errors [4]. The percent error for the even harmonics are actually negative in sign but are presented on the same graph for the reader's convenience.

The measurement errors for the Type A meter, a charge sensing device (Eq. 3), can be determined directly from the curves shown.

There are additional considerations which enter into the calculations in the case of the Type B field meter. As noted in Section II.A, this device responds to the current induced (Eq. 4) between its sensing electrodes. The current results from the capacitive coupling between the transmission line and the field meter and between the field meter and ground. Since these admittances are directly proportional to frequency, the amplitude of each harmonic component of current induced between the sensing plates of the field meter relative to the amplitude of the fundamental is increased by the order of the harmonic, e.g., by a factor of 3 for the third harmonic, a factor of 5 for the fifth harmonic, etc. An RC network introduced in the signal processing portion of the Type B 
field meter modifies the relative amplitudes and phase relationships of the harmonic components; however, since the network is not the same for all models it is not possible to calculate error curves which apply to all Type B instruments. The curve shown in Figure 2 indicates the calculated percent error for the third harmonic if the RC network is ignored.

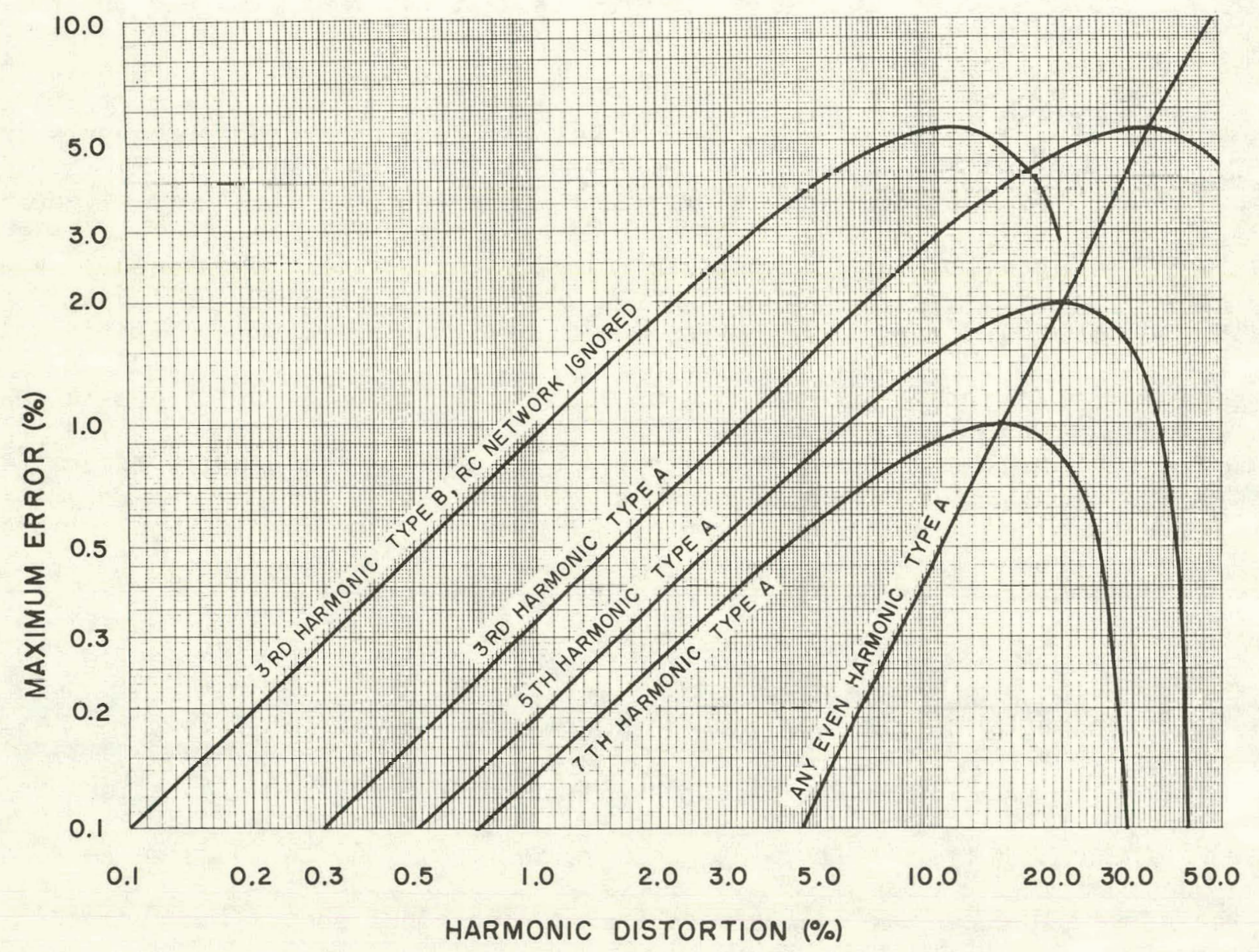

Figure 2. Maximum rms value errors due to selected harmonic components for Type A and $B$ meters. The even harmonic percent errors are actually negative in sign. The error curve for the Type B meter dons not. include the effect of the RC network (see text).

The following experimental data obtained with voltage waveforms having measured harmonic distortion (but with unknown phase relationships between fundamental and harmonic components) provide confirmation that the curves of Figure 2 provide an indication of the error which may be encountered. 
Error in Field Measurement

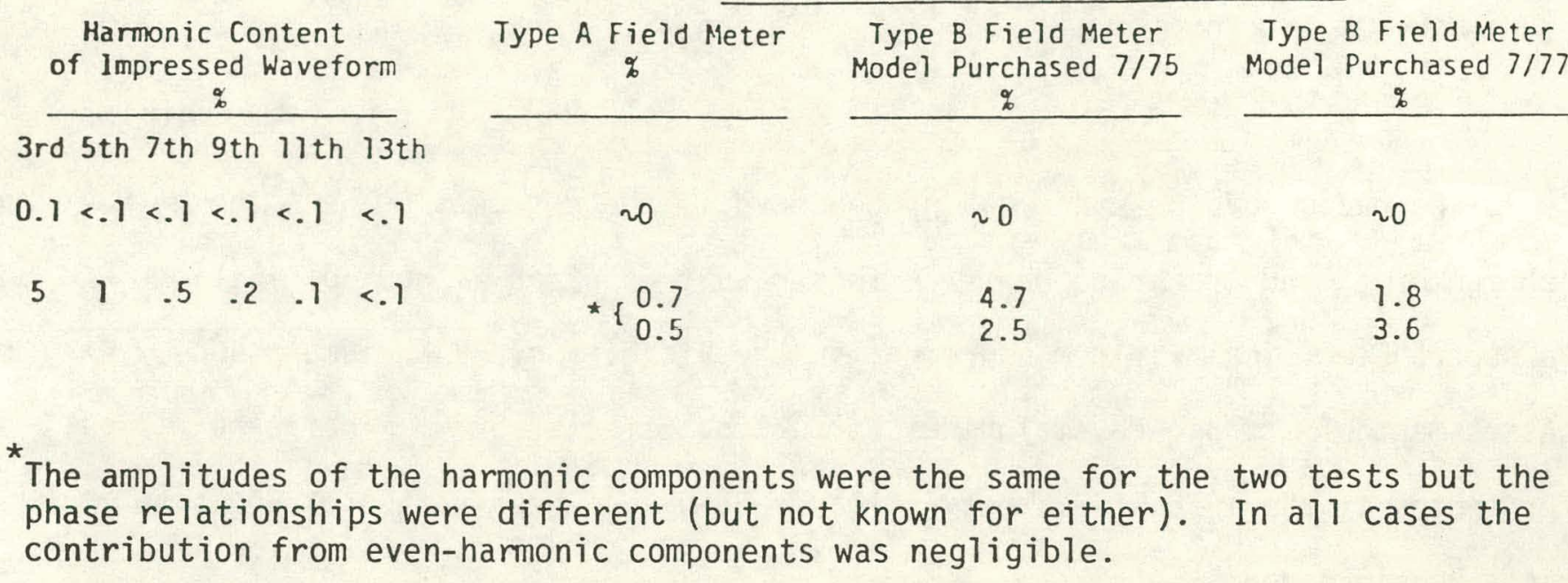

Under normal operating conditions in most locations under transmission lines the harmonic content in the electric field waveform is probably in the range of $1-2 \%$ or less; however, close to large industrial loads it may reach $10 \%[5]$ and thus may introduce significant errors in field strength measurements.

\section{(g) Measurement of Non-uniform E-fields}

A thesis by Lothar Wilhelmy of Stuttgart University [6] describes the design and operation of an electric field probe which can be used in nonuniform as well as uniform electric fields. The magnitude and direction can be measured even in the presence of rapid field fluctuations. The device is considerably more complicated and expensive to fabricate than the commercially available U.S. meters.

Although designed for measurements of near-uniform fields, the U.S. meters may have application in some relatively non-uniform field situations as the following theoretical analysis suggests. The current induced in a spherical E-field probe in a non-uniform quasistatic field generated by a point charge is given by (in the absence of nearby ground planes)

$$
I=3 \pi a^{2} \omega \varepsilon_{0} E(\omega t)\left[1-\frac{7}{12}\left(\frac{a}{d}\right)^{2}+\frac{11}{24}\left(\frac{a}{d}\right)^{4}-\ldots\right] \ldots
$$


where

$$
E(\omega t)=\frac{1}{4 \pi \varepsilon_{0}} \frac{Q(\omega t)}{d^{2}}
$$

a is the radius of the spherical probe and $d$ is the distance between the point charge $Q(\omega t)$ and the probe center. This result is given without derivation in a paper "Electrical Field Measurement in the Vicinity of HV Equipment and Assessment of Its Bio-Physiological Perturbing Effects" by C. Mihaileanu et. al., presented at the 1976 CIGRE Meeting (Paper 36-08). A derivation of Equation 5 is given in Appendix $A$.

The current induced in a spherical E-field probe in a uniform quasistatic fjeld, $E_{0}(\omega t)$, is given by [see Eq. 4]

$$
I=3 \pi a^{2} \omega \varepsilon_{0} E_{0}(\omega t) .
$$

Comparison of Equations 5 and 6 shows that the induced current for the nonuniform field case is the same as that produced by a uniform field of magnitude $E_{0}=Q / 4 \pi \varepsilon_{0} d^{2}$ if the terms in $(a / d)$ can be neglected. Thus the induced current at a point in the highly non-uniform field produced by a point charge is nearly the same as that produced by a uniform field of equal magnitude if $d$ is sufficiently large. For example, if $a / d=0 . I$, the difference in induced current (E-field measurement) produced by a uniform and highly non-uniform field is less than $1 \%$; the change in E-field magnitude over the dimensions of the sphere is $|\Delta E / E| \simeq 4 \mathrm{a} / \mathrm{d}=0.4(40 \%)$.

The "effective" radius of commercially available E-field meters, which have rectangular geometries, can be estimated as half of the largest diagonal dimension. It would, therefore, appear that these instruments can be used with slightly reduced accuracy in regions where the field nonuniformities approximate that of 
the field of a point charge at a distance of five times the major dimension of the field meter.

To examine this theoretical prediction, a non-uniform field was produced by energizing an aluminum sphere (15.2 cm diam.) suspended $2 \mathrm{~m}$ above a concrete floor. The experimental arrangement is shown in part in Figure 3. The E-field at several locations below the sphere was measured with $\sim 3 \%$ accuracy using a miniature E-field probe developed at NBS (Appendix D). The field was also measured using both types of commercial meters. The E-field values measured with the various devices are plotted in Figure 4 as a function of distance from the sphere's center and field meter center. All the instruments were calibrated in a uniform field between parallel plates (Section II.C.3). No corrections have been made for temperature differences at the time of calibration and during the non-uniform field measurements (this could lower the datum points of Type $A$ and $B$ meters by $\sim 1 \%$ to $2 \%$ ). Errors in reading the analog output of the commercial meters during the experiment are estimated to be less than $\sim 3 \%$.

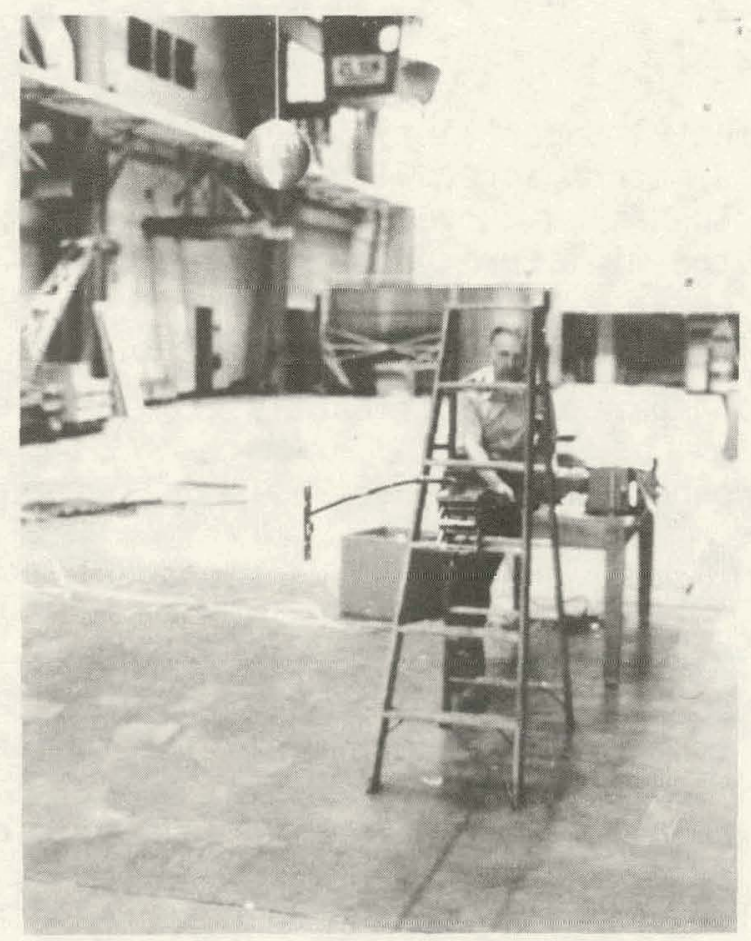

Figure 3. Experimental arrangement for generating a non-uniform electric field. 


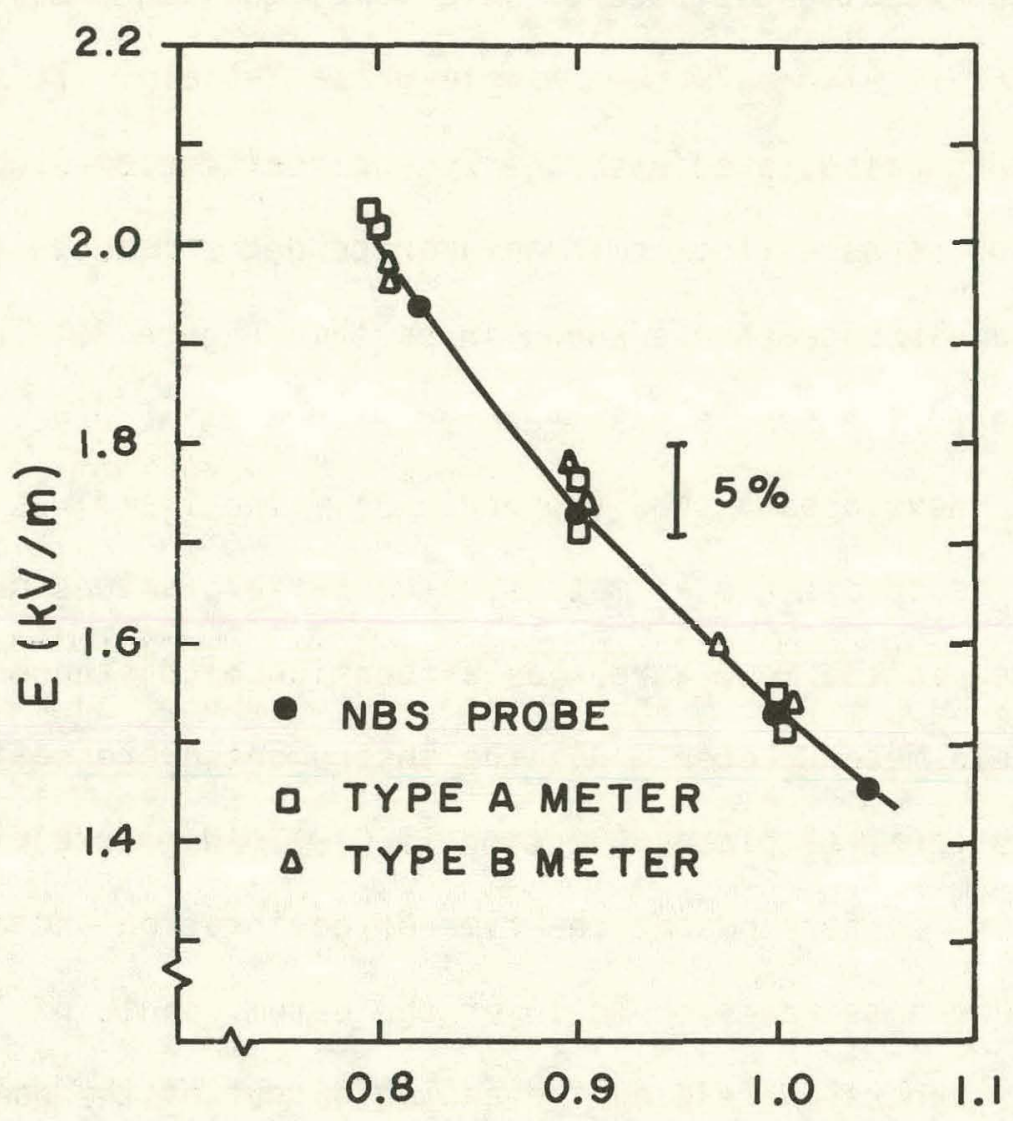

DISTANCE FROM SPHERE CENTER $(\mathrm{m})$

Figure 4. Within experimental uncertainty, the field measurements obtained with the large field meters agree with those observed with the miniature field probe. The solid curve has been drawn to pass through the points measured with the miniature probe.

The non-uniform field departed significantly from that expected for a point charge over an infinite ground plane because, as modeling experiments showed, contributions to the field occurred from the unshielded lead which energized the sphere.

The data of Figure 4 confirm the theoretical expectation that, within experimental uncertainty, relatively large commercial E-field meters which are calibrated in uniform fields can also be used to measure non-uniform fields ( 29\% change over dimensions of Type B meter) with introduction of small error. 
The theoretical and experimental results have been obtained under the constraint that the meter is aligned with a single-phase E-field. It should be noted that the asymmetry associated with the Type B field meter (II.B.1.d) imposes a requirement for particular care in interpreting the results obtained with it in non-uniform field situations.

\section{Field Studies}

\section{(a) Effect of Proximity of the Observer}

Limited studies of the proximity effect of the observer were conducted under the "C" Iine at the Apple Grove, West Virginia EHV test facility and under an operating $500 \mathrm{kV}$ line near Urbana, Maryland. The meters, with handles attached, were supported on a glass tube and read with the aid of field glasses from a distance of approximately 7 meters by one observer as another observer stood at measured distances from the instrument.

The extent and nature of the field distortion introduced depends on many variables in addition to the horizontal distance between the meter and the observer, i.e., (a) the detailed geometry of the transmission line; (b) the relative positions of the line, meter, and observer; (c) how well the observer is grounded; (d) the effective height of the "ground plane"; (e). the height of the observer; and ( $f$ ) the height of the field meter above the ground. Table II provides an indication of the influence of some of these parameters on measurements under the $500 \mathrm{kV}$ line.

Comparisons of the perturbation caused by well-insulated observer with those caused by one who was well grounded revealed that the effect was noticeably larger for the grounded observer at distances where the perturbation was significant. The data reported here were taken with the "observer" wearing conducting shoes and holding a wire attached to a $40 \mathrm{~cm}$ metal rod driven into the ground. Thus from this aspect they represent a worst case situation. 


\section{TABLE II}

Effect of Proximity of Six-foot Observer

(field meter one meter above ground)

\begin{tabular}{|c|c|c|c|}
\hline Meter & $\begin{array}{c}\text { Meter } \\
\text { Location } \\
\end{array}$ & $\begin{array}{l}\text { Handle Orientation } \\
\text { Relative to } \\
\text { Iransmission Line } \\
\end{array}$ & $\begin{array}{c}\text { Approximate } \\
\text { Meter-Observer } \\
\text { Distance Resulting } \\
\text { in }-5 \% \text { Error } \\
(\mathrm{m})\end{array}$ \\
\hline$A$ & $\begin{array}{l}\text { Under outer } \\
\text { conductor }\end{array}$ & Parallel & 2.0 \\
\hline$"$ & $"$ & $\begin{array}{l}\text { Perpendicular, } \\
\text { toward outside }\end{array}$ & 2.1 \\
\hline$"$ & $"$ & $\begin{array}{l}\text { Perpendicular, } \\
\text { toward center line }\end{array}$ & 1.8 \\
\hline B & $"$ & Parallel & 1.4 \\
\hline$"$ & $"$ & $\begin{array}{l}\text { Perpendicular, } \\
\text { toward outside }\end{array}$ & 2.0 \\
\hline$"$ & $"$ & $\begin{array}{l}\text { Perpendicular, } \\
\text { toward center line }\end{array}$ & 1.4 \\
\hline$"$ & $\begin{array}{l}\text { Under center } \\
\text { conductor }\end{array}$ & Parallei & 1.5 \\
\hline$"$ & $"$ & Perpendicular & 1.4 \\
\hline
\end{tabular}

*observer proximity effects which result in an error of opposite sign are considered in the next section. 
It was found that the proximity effect of the observer is significantly different for the two types of field meters as Table II and Figures 5 and 6 show. These data, which are representative of the data obtained with other configurations, were obtained with the field meter under the center conductor and with its handle oriented in a direction perpendicular to the line. The area was covered with grass about $10 \mathrm{~cm}$ high and was reasonably flat with only a slight slope.

The results shown in Figures 5 and 6 indicate that the error introduced by the proximity of the observer couid be reduced by specifying a height above ground for field measurements greater than one meter. Unfortunately, the optimum height is not the same for the two field meters. It appears to be about 1.5 meters for the Type $A$ and 1.2 meters for the Type $B$.

An explanation for the differences between the proximity effects with the two field meters is to be found in the geometric asymmetry in the Type B field meter sensing electrodes (see Section.II.B.1.d). While this departure from symmetry imposes the necessity for the precautions mentioned in Sections II.B.1.b and II.B.1.d, it does result in a reduction in the perturbing effect of the observer for a range of field meter heights.

(b) Comparison of U.S. and Soviet E-field Measurement Procedures

Electric field measurements in the U.S.S.R. have been made at a height of 1.8 meters [7] using a field meter with a $25 \mathrm{~cm}$ handle $[8,9]$. Field measurements in the U.S. are normally made at a height of $1 \mathrm{~m}$ with considerably longer handles $(\sim 1 \mathrm{~m}$ to $\sim 3 \mathrm{~m}$ ). Interest, first generated in 1975 [10], has recently focused on obtaining a relationship between E-field values measured in the two countries. Attempts at simulation of the Soviet measurement technique have been made in the U.S. by holding, $1.8 \mathrm{~m}$ high with outstretched arm, commercial U.S. meters with a shortened grip on the handle. The field values obtained in this fashion are compared with values obtained at the same location 


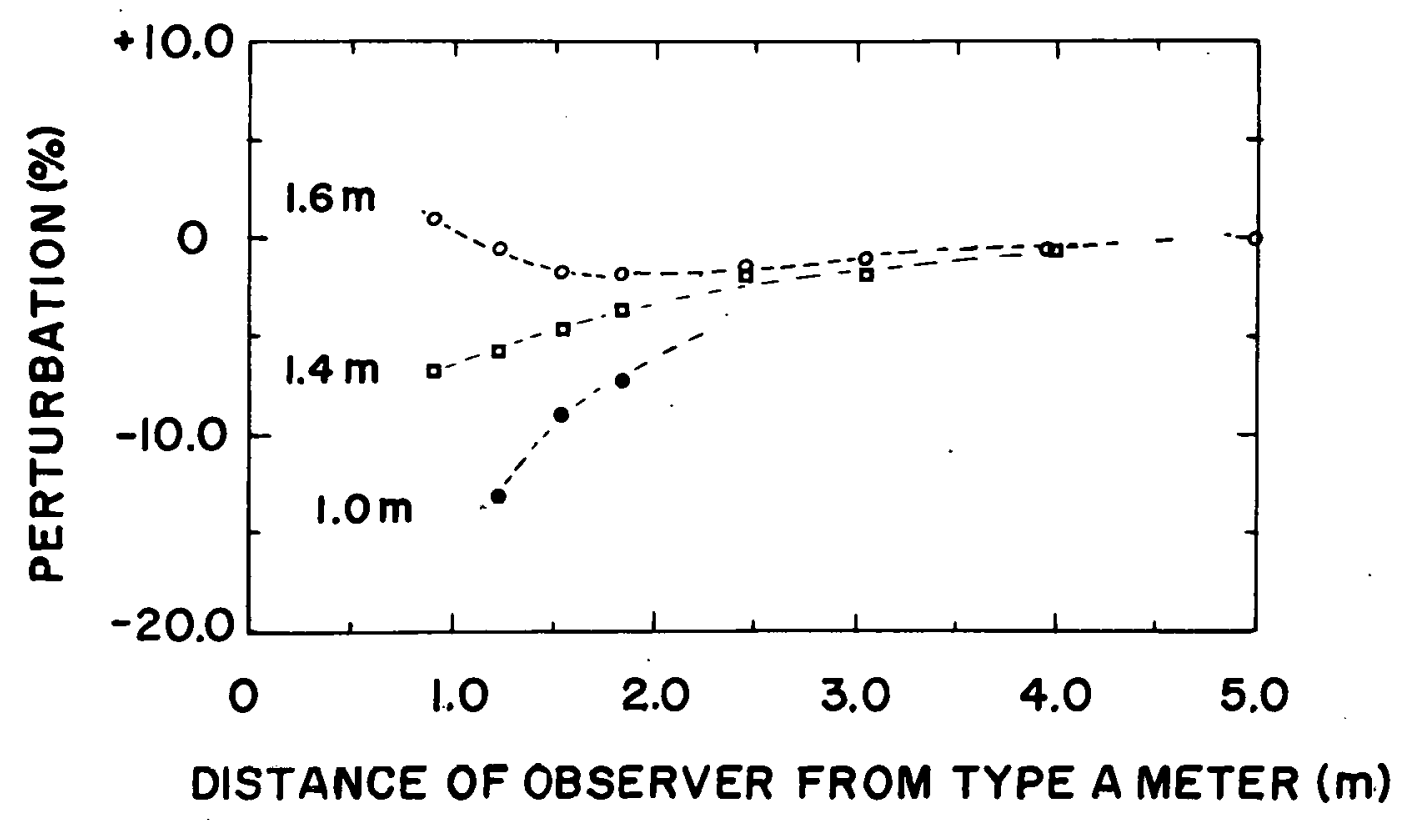

Figure 5. Proximity effect of six foot observer for Type A Field Meter at heights of $1.0,1.4$, and $1.6 \mathrm{~m}$.

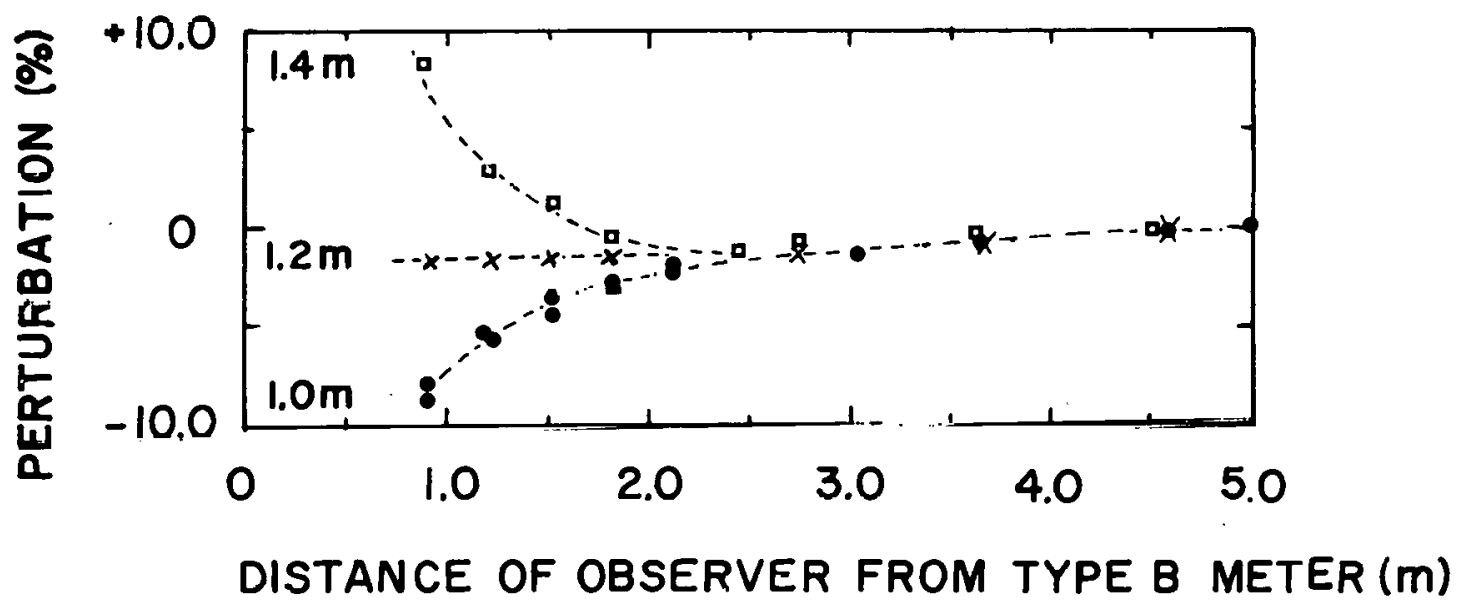

Figure 6. Proximity effect of six foot observer for Type B Field Meter at heights of $1.0,1.2$, and $1.4 \mathrm{~m}$. The two sets of data for the $1.0 \mathrm{~m}$ height give an indication of the reproducibility of the results. The proximity effect of bystanders positioned in directions other than along the handle.will introduce greater perturbations for the $1 \mathrm{~m}$ height. 
$1 \mathrm{~m}$ above the ground with a long handle. The ratio of the two values has been found by a number of observers to range from approximately 1.2 to 1.8 depending on the geometry of the meter-observer-transmission line combination and the choice of commercial meter (Type A and Type B).

Such measurements were repeated under a $500 \mathrm{kV}$ transmission line near Urbana, Maryland using both Type A and Type B meters. At a location of about 3.5 m outside the outer phase conductor (near maximum E-field) the ratio of the "Soviet" E-field value (short handle - $1.8 \mathrm{~m}$ ) to U.S. E-field value (long handle $-1 \mathrm{~m}$ ) was found to be about 1.8 using a Type B meter and 1.4 using a Type A meter. The ratios changed to about 1.7 and 1.3 respectively when the location was changed to about 14 meters outside the outer pliase conductor. It should be noted that the principal cause of the observed differences (1.8 $\mathrm{m} \mathrm{vs} .1 \mathrm{~m})$ is the perturbation introduced by proximity of the observer rather than differences in elevation above the ground.

The lack of agreement between ratios obtained with Type $A$ and Type $B$ meters has a qualitative explanation which raises questions regarding the accuracy of such simulation experiments. As noted above, the enhanced field value at $1.8 \mathrm{~m}$ is primarily due to the proximity of the observer and his hand. The observer may be regarded as a nearly grounded object which concentrates the field and it is this enhanced field the meter "sees." Depending on the geometry of the hand-handle combination relative to the sensing plates, the geometry of the meter sensing electrodes (i.e., flat plates or "hemispheres"), the relative position of the transmission lines and perhaps other parameters (e.g., closeness of meter to body, height of observer) the enhancement (arid ratio) will be different. Figure 7 shows the approximate geometries of the Soviet meter $[8,9]$ and the two U.S. meters; arrows indicate field sensing surfaces. Marked differences can readily be seen which, when taken with the asymetry characterizing the Type $B$ 

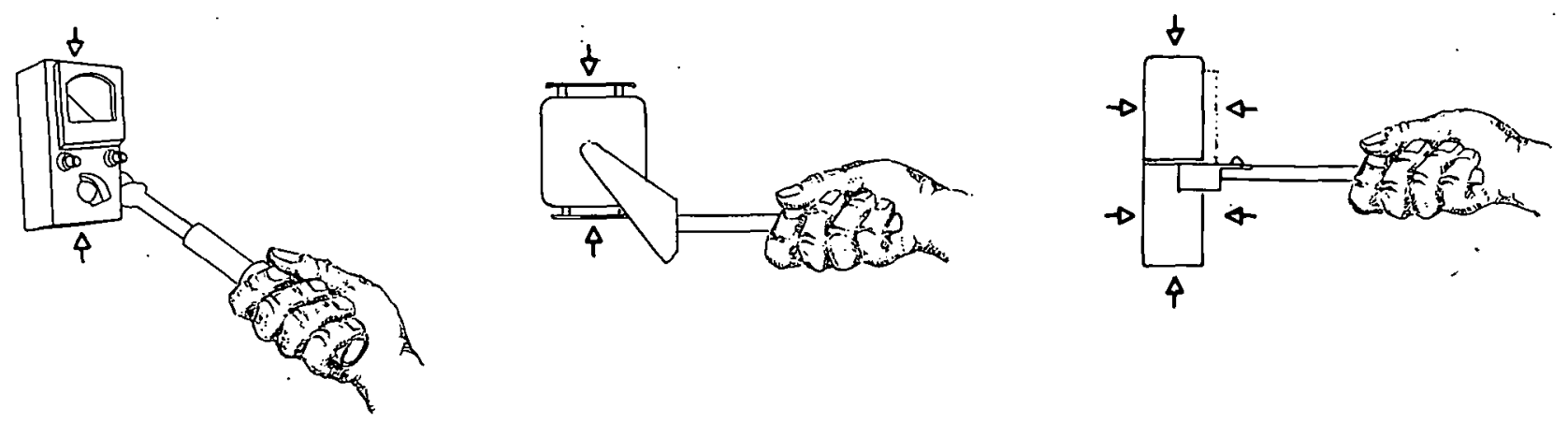

Figure 7

(Figures are not scale drawings)

meter (see Section II.B.1.d), can provide an explanation for the observed results.

The above observations were confirmed qualitatively by suspending U.S. field meters in a parallel plate E-field and hringing a grounded aluminum foil "hand" to within $25 \mathrm{~cm}$ of the meter. The enhancement in field reading was found to depend on direction of approach and choice of field meter.

The lack of documentation providing details of the Soviet measurements (e.g., closeness of meter to body, orientation of body with respect to signal source, height of observer, etc.) as well as the lack of availability of the appropriate Soviet meter makes any comparison of measurement techniques difficult. What seems certain from the above comments is that the measurements made using the Soviet technique will be significantly larger than U.S. measurements because of proximity effects. (The reader should note also that the proximity cffcct of the observer can decrease the field reading when the meter is held at the lowered height of $1 \mathrm{~m}$. See section II.B.2.a). Because different ratios are obtained with the same field meter at different locations relative to the transmission line, the ratio will have a range of values (presumably this would be true even if the Soviet field meter were used). It is also perhaps noteworthy that the quoted [9] accuracy of the Russian field meter is $\pm 20 \%$. 


\section{Calibration of Electric Field Meters}

\section{Introduction}

Calibrations of ac electric field meters have been made in quasi-static $(60 \mathrm{~Hz})$ uniform fields of known ( $r m s)$ magnitude. A uniform field has been chosen because it resembles the transmission line fields usually encountered near ground level. (Measurements of transmission line fields are usually performed at a height of 1 meter). The small non-uniformities which actually exist in transmission line fields near ground level are not expected to influence the E-field measurement in light of the discussion of Section II.B.l.g.

Two methods of calibration have been used: (1) A uniform field is established under an energized line and the current through a guarded electrode of known area (see "one meter square panel" Appendix C) placed at ground level is measured to determine the field value. The field meter to be calibrated is supported at a distance of $\sim 1 \mathrm{~m}$ above the electrode. The field at the instrument is assumed to be the same as that at the electrode. (2) The field meter is inserted into the space between two parallel-plate electrodes having a known separation and a voltage applied between them. The field is assumed uniform over a volume large compared with the dimensions of the instrument prior to its insertion.

While sometimes improperly referred to as an alternative method of calibration, the current injection technique described in Section II.C.5 provides a calibration of the signal processing circuits of the field meters and thus serves as a very useful calibration check.

Since it is believed that the convenience offered by the parallel-plate calibration technique relative to the "one square meter panel" method will. lead to its adoption by most users of field meters, a rather extensive theoretical and 
experimental study has been made of factors which influence the accuracy obtainable with this method.

2. Finite Size of Plates and Fringing Fields

Uniform field regions of known magnitude and direction can be created with parallel plates provided that the spacing of the plates, relative to the plate dimensions, is sufficiently small. The uniform field value $E_{0}$ is given by $V / t$ where $V$ is the applied voltage and $t$ is the plate spacing.

While many texts have treated the problem of semi-infinite plates theoretically, a recent Sandia report by $P$. D. Thacher [11] has presented the results in perhaps the most graphic form. In common with earlier analytical treatments, the problem is solved by utilizing the theory of functions of a complex variable [12]. The details of the conformal mapping which permits the problem's solution are not presented here. They are provided in the references cited.

The results of Thacher's calculation are shown in Figure 8 with the author's kind permission. Illustrated are contours of equal field intensity relative to the uniform value squared, $E^{2}=v^{2} / t^{2}$. Onily one electrode is shown with the midplane. of the electrodes corresponding to the bottom of the figure. The coordinates $u^{\prime}$ and $v^{\prime}$ are shown in the inset. The magnitude of the relative electric field is found by taking the square root of the numbers shown. It should be noted that the field direction is not explicitly shown. It can be assumed, however, that when the field magnitude is nearly equal to the uniform value, the direction will be approximately normal to the plates.

Using the results of Figure 8 , the magnitude of the electric field at either plate surface and the midplane can be plotted as a function of distance from the plate edge. This is shown in Figure 9 with numerical values [1I] shown in lable 111. Thus given a plate spacing $t$, one can determine how large the plates must be in order to have field uniformity to a specified degree. For the idealized case of 
no nearby planes or objects, these results could be used to design a finite-sized parallel plate structure. A linear superposition of the edge effects (nonuniformities) due to opposite edges of the plates can be made at the center when the perturbations are less than approximately $0.5 \%$.

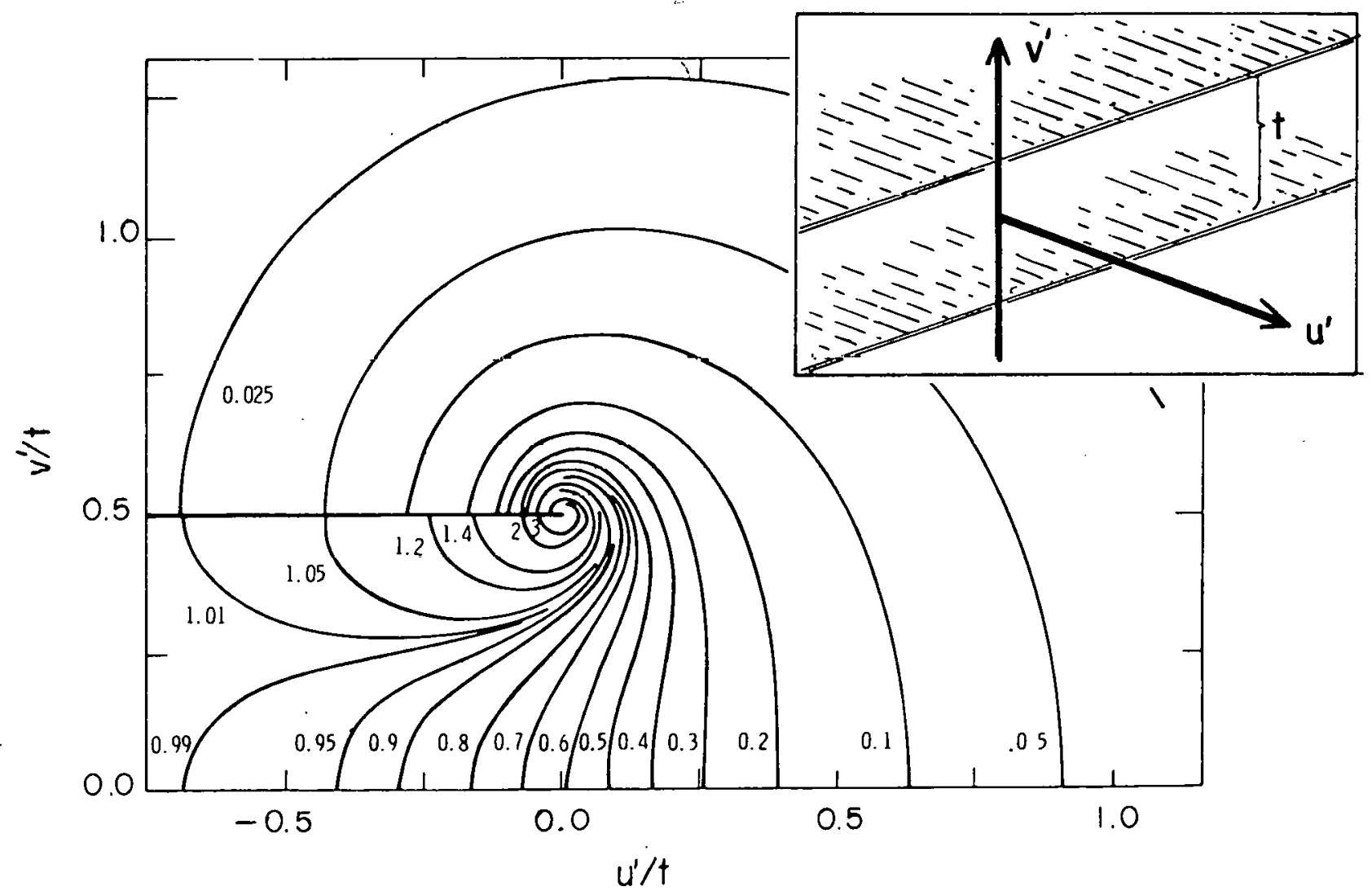

Figure 8. Contours of equal field intensity. Only one electrode is shown, with the bottom of the diagram corresponding to the midline between electrodes. $v a l u e s$ of ' $u^{\prime}$ and $v^{\prime}$ are normalized by the electrode separation $t$. Numbers beside the 7 ines give the field squared relative to $\mathrm{V}^{2} / \mathrm{t}^{2}$, the value well in from the edge of the electrodes.

3. Modification of Fringing Fields, Compensating Procedures and Generation of a Calibration Field

As noted in the previous section, the theoretical results presented for the design of a parallel plate calibration structure are valid in the absence of nearby ground planes (walls, floor, etc.) and other perturbing objects. The degree of 


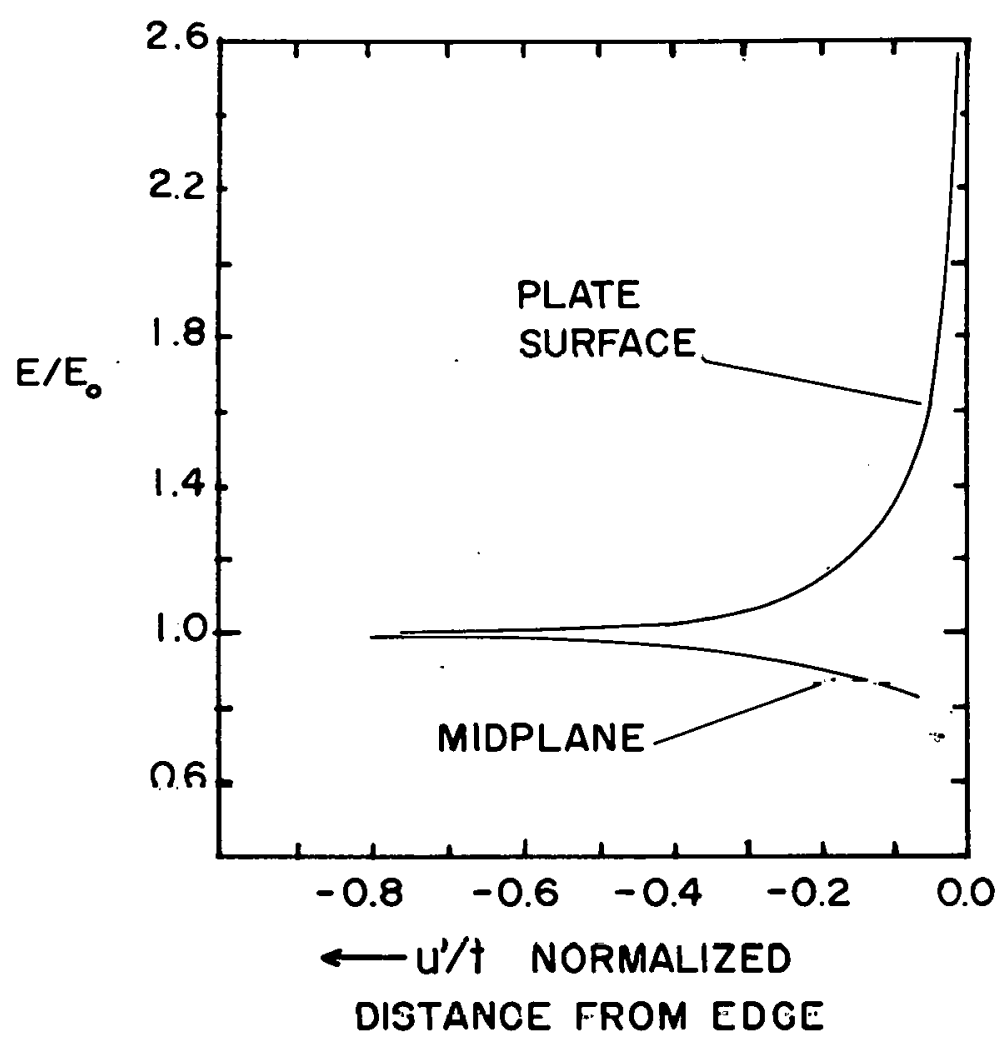

Figure 9. Variation of normalized electric field strength at plate surfaces and midplane as function of normalized distance from plate edge.

modification of the theoretically predicted E-field will depend on the geometry of these surfaces as well as the method used to energize the parallel plates. The two methods commonly used to apply a voltage to the plates are (a) "balanced" arrangement in which a grounded center-tapped transformer is employed to provide voltages on the two plates equal in magnitude but $180^{\circ}$ out of phase, and (b) "single-ended" arrangement in which one plate is grounded. The balanced scheme was chosen as the better arrangement because modeling experiments demonstrated that there was a greater degree of immunity from the perturbing influences of nearby ground planes. Significant departures from idealized surface E-field predictions (Table III) were evident using a single-ended arrangement, even when the nearby ground planes were several plate spacings away. Details of the modeling measurements are presented in Appendix $B$. The effectiveness of guard rings in reducing the perturbation and creating a larger region of uniform field is also examined in Appendix B; guard bands are discussed later in this section. 
Table III

MIDPLANE

$u^{\prime} / t$

$-0.0698$

$-0.1621$

$-0.2965$

$-0.4177$

$-0.6821$

$-0.7934$

$-0.7954$

$-0.6861$

$-0.4376$

$-0.2431$

$-0.1624$

$-0.1230$

$-0.0991$

$-0.0829$

$-0.0452$

$-0.0307$

$-0.0185$
$\underline{\left(E / E_{0}\right)}$

0.836

0.894

0.948

0.974

ก. 994

0.997

PLATE SURFACE

1.002

1.005

1.025

1.095

1.183

1.265

1.341

1.414

1.732

2.000

2.449 
Using the results of the previous section, it can be shown that, in the absence of external perturbations, the E-field between parallel plates $3 \mathrm{~m} \times 3 \mathrm{~m} \times 1 \mathrm{~m}$ spacing can reach values that are within a few tenths of a percent of the uniform value $V / t$. A structure of this size was constructed for purposes of investigation and is shown in Figure 10. Both plates consist of nine tightly stretched aluminum screens, $1 \mathrm{~m} \times 1 \mathrm{~m}$, mounted on an aluminum frame. The top plate is suspended from the ceiling with electrically isolated chains and turnbuckles; the bottom plate is mounted on wooden tables about $1 \mathrm{~m}$ above the floor. The nearest wall is located about $1.5 \mathrm{~m}$ away. The plate flatness and spacing was adjusted to reduce the uncertainty in plate spacing to less than $\sim 0.5 \%$.

The power supply consists of a low distortion oscillator-amplifier combination supplying two transformers with primary windings in parallel, and secondary windings in series "center-tapped" to ground. The total harmonic content of the transformer

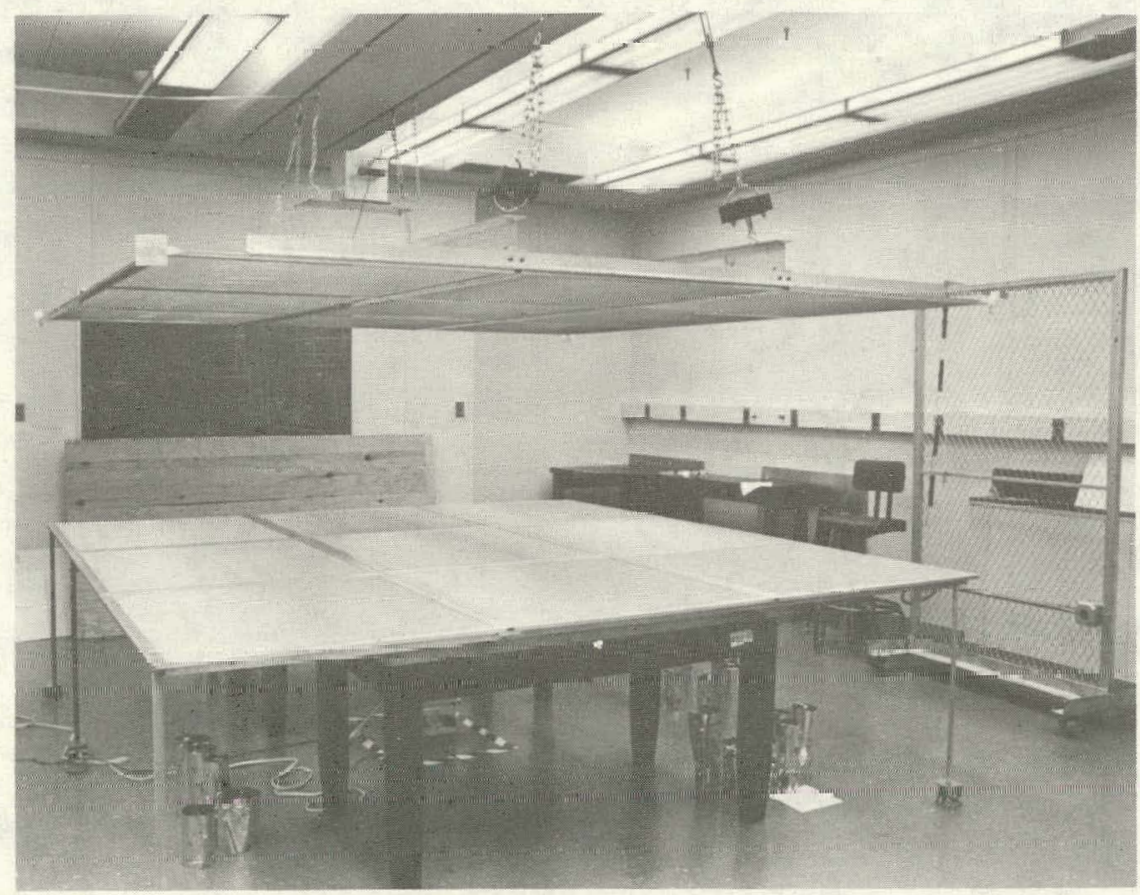

Figure 10. Large parallel plates with $1 \mathrm{~m}$ separation. Each plate consists of nine $1 \mathrm{~m} \times 1 \mathrm{~m}$ tightly stretched aluminum window screens mounted on an aluminum frame. 
output voltage is less than $1 \%$. As a safety measure, three-megohm current limiting resistors are placed in series with the output of each transformer. The voltage difference across the plates is measured with a calibrated electrostatic voltmeter with an error less than $\sim \pm 0.2 \%$. The system remained corona free during the measurements described below.

Measurements of the vertical and horizontal E-field profiles were made with a Type B field meter. The meter was inserted into the field with a dielectric rod, $2 \mathrm{~m}$ long, which was mounted on a wooden frame. Readings of the analog output were made with a cathetometer aligned to reduce parallax; differences of less than $1 \%$ could be discerned. Figure 11 shows the vertical E-field component as a function of height near the center of the plates. The horizontal profile of the vertical E-field at a number of heights above the bottom plate are shown in Figure 12. The inset in Figure 12 compares the horizontal profile at a height of $50 \mathrm{~cm}$ with the predictions of Section II.C.2 ignoring superposition of fringing fields. The field values in Figure 11 and the inset of Figure 12 have been normalized with respect to the value measured at the center of the parallel plates.

The data of both figures indicate that a substantial region of nearly uniform field exists between the plates. The vertical profile shows that, within experimental error $(\approx 1 \%), E$ is constant from $\sim 30 \mathrm{~cm}$ to $\sim 70 \mathrm{~cm}$ above the bottom plate. The increase in field values at distances closer to the plates can be attributed to interaction between the meter and surface charge distributions on the plates (Appendix B.i). Thus it can be assumed that $E$ remains constant along the entire vertical path. Since the potential difference $V$ must equal the line integral

$$
V=\int_{0}^{t} \vec{E} \cdot d \vec{l}=E \int_{0}^{t} d l=E t
$$




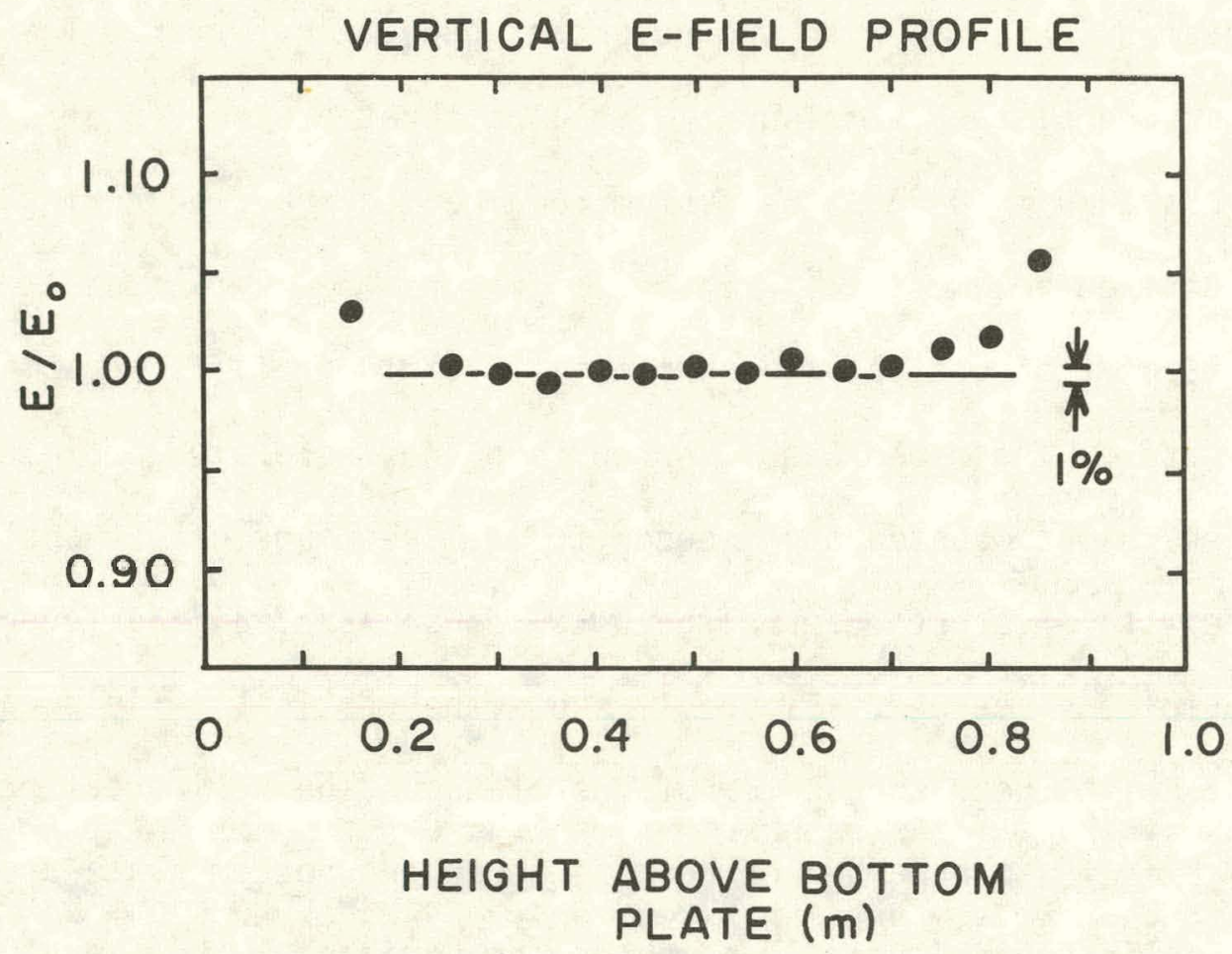

Figure 11. Within experimental uncertainty, a region of nearly constant E-field is observed from $\sim 30 \mathrm{~cm}$ to $\sim 70 \mathrm{~cm}$ above the bottom plate.

it is possible to conclude that $E$ is within $\sim 1 \%$ of the uniform value $V / t$ and that a structure $3 \mathrm{~m} \times 3 \mathrm{~m} \times 1 \mathrm{~m}$ will generate a nearly uniform field.

This conclusion regarding field uniformity was confirmed with a second independent experiment employing guard bands. Both guard bands and rings (Appendix B.iii) have been used to enlarge the region of uniform field between parallel plates. Guard bands have been used extensively in the past for calibration of $x$-ray beams, and, consequently, the relevant theory and experimental verification can be found in the literature [13].

The spacing of the large parallel plate structure was increased to $1.2 \mathrm{~m}$ and aluminum foil ( $23.8 \times 10^{-3} \mathrm{~cm}$ thick) bands $14 \mathrm{~cm}$ and $28 \mathrm{~cm}$ wide were wrapped around the perimeter as shown in Figure 13. Bakelite tubes at the corners and masking 


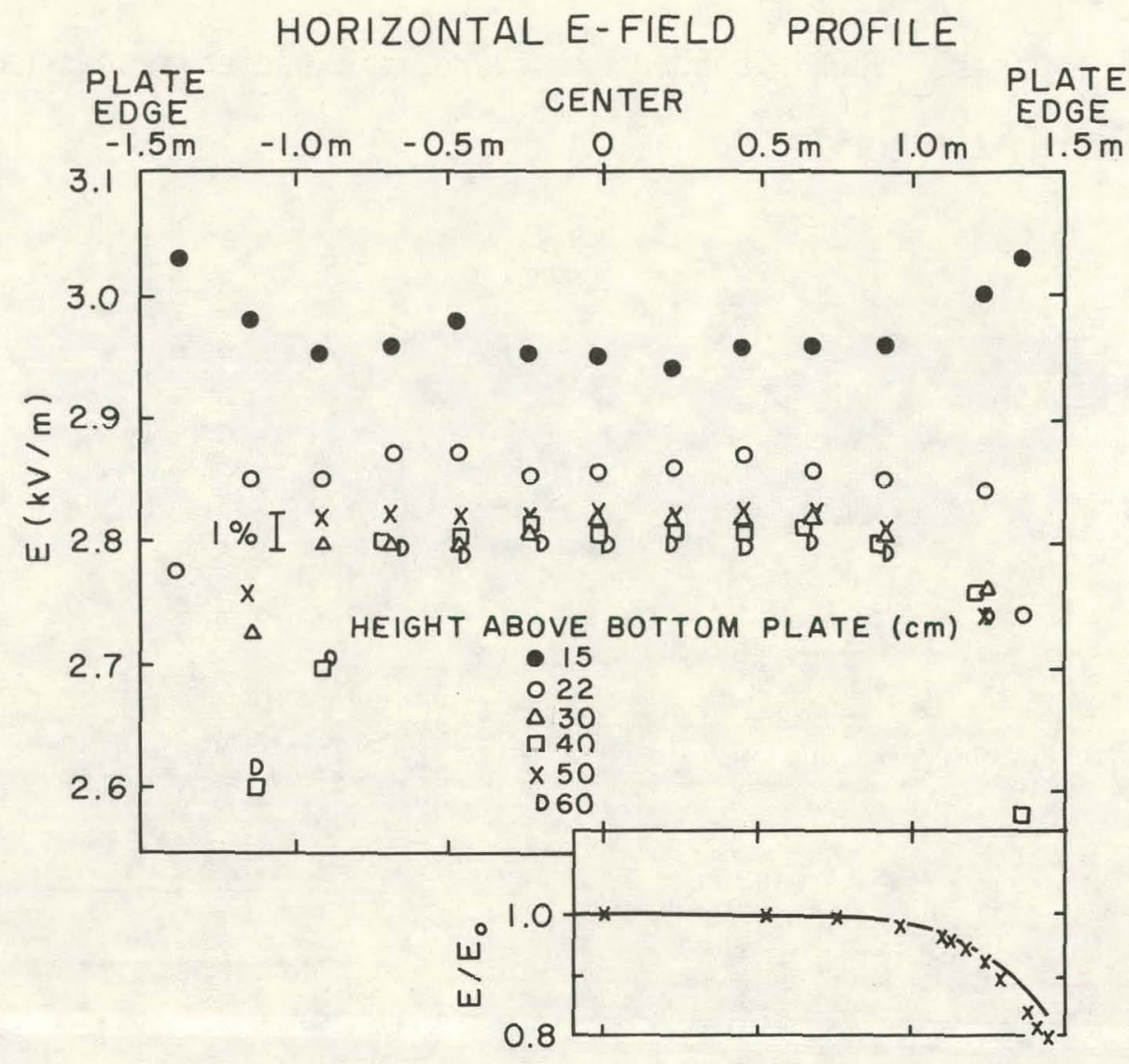

Figure 12. The horizontal profile of the vertical E-field measured at several heights above the bottom plate. The inset compares experimental observations of the normalized field at $50 \mathrm{~cm}$ with the theoretical predictions for semi-infinite plates. The presence of ground planes less than two plate spacings away from the apparatus and the superposition of fringing fields do not significantly perturb the field from $\sim-0.75 \mathrm{~m}$ to $+0.75 \mathrm{~m}$.

tape at intermediate points were used to support the bands. A resistor divider of four 5-megohm resistors was used to grade the potentials applied to the bands. The spacing between adjacent bands was nominally $2 \mathrm{~cm}$ which is sufficiently large to avoid corona and sufficiently small for application of the experimentally confirmed theory [13]. The bottom plate was grounded to allow deployment of a current-sensing probe on the bottom plate. The earlier concern regarding the perturbing influence of nearby ground planes when using the single-ended arrangement 
was not applicable in the present case because of the large electrical isolation provided by the bands. Such a banded structure is expected to generate a large volume of uniform field [13].

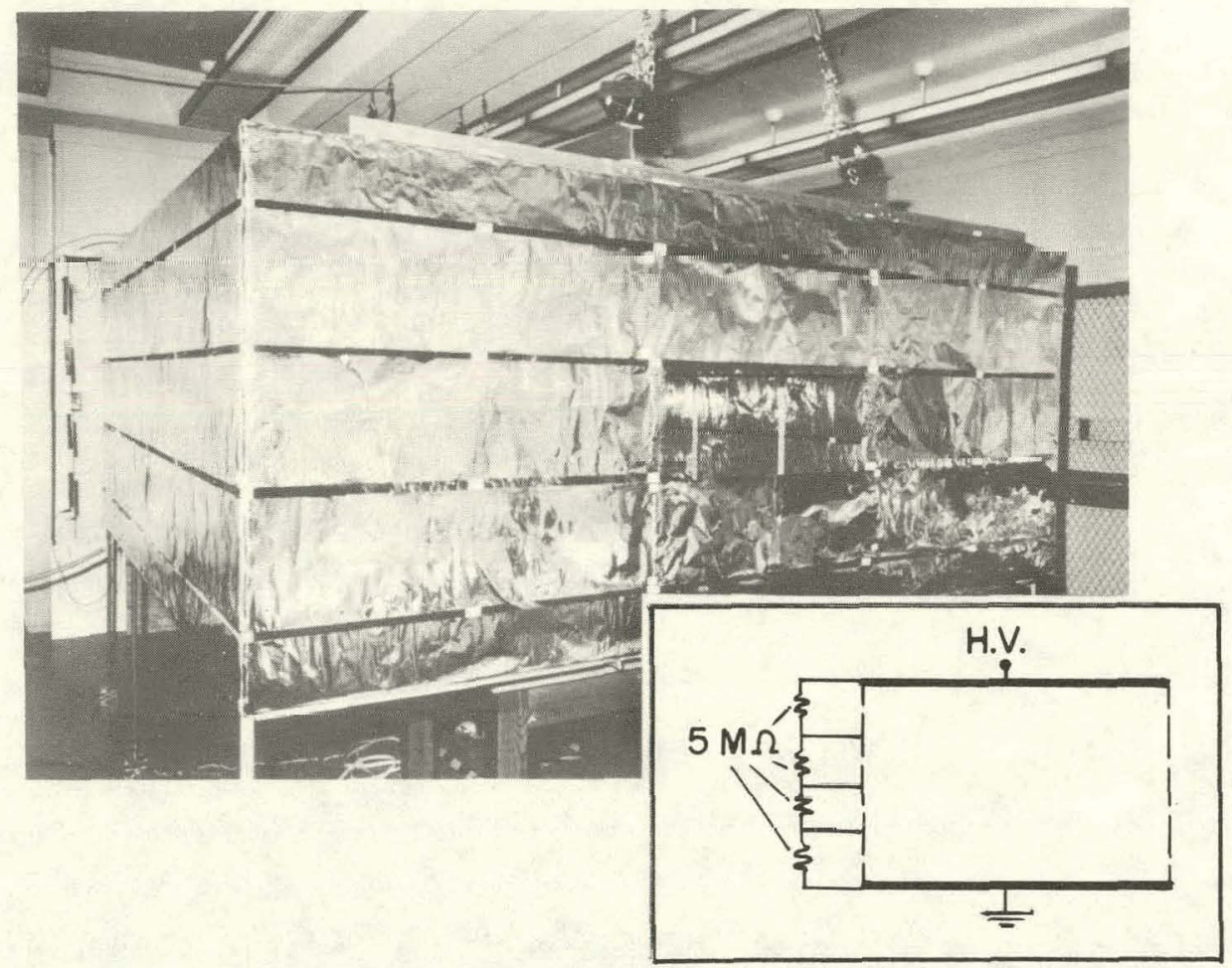

Figure 13. Large parallel plates with guard bands. E-field meters are introduced into the field via the "window" in the band structure. The inset is a schematic view of resistor divider and electrical connections.

The current-sensing probe consisted of an aluminum covered $0.15 \mathrm{~cm}$ thick plastic disc $16.5 \mathrm{~cm}$ in diameter. An isolated circular segment, $8.5 \mathrm{~cm}$ in diameter, in the center made up the current sensing surface.

Measurement of the induced current (see Appendix B.i) permitted determination of the E-field along the bottom plate to within an accuracy of $\pm 0.2 \%$.

Values of the E-field along the surface of the bottom plate are shown in Figure 14. The data have been normalized with respect to the uniform field value 
$\mathrm{V} / \mathrm{t}$. The lack of symmetry is due in large part to an opening in the band structure (Figure 13). The results show that a uniform field does exist across much of the bottom plate surface confirming our expectations; it can be inferred that this field extends nearly vertically to the top plate [13].

The earlier conclusions regarding the field uniformity of the unbanded $3 \mathrm{~m} \times 3 \mathrm{~m} \times 1 \mathrm{~m}$ structure were confirmed with the banded structure $(3 \mathrm{~m} \times 3 \mathrm{~m} \times 1.2 \mathrm{~m})$ in the following way. E-field measurements were first made at the-center of structure with both types of field meters. The bands were then removed and the same voltage difference was applied to the plates using a balanced arrangement. The E-field measurements were repeated. No measurable differences could be seen in the field values indicating a uniform field for both configurations. Reducing the plate spacing from 1.2 to $1 \mathrm{~m}$ increases the lateral region of field uniformity.

Having constructed a relatively large apparatus which generated a uniform E-field, the question of building a smaller more easily fabricated system for calibration purposes was addressed. With the goal of producing a field which was within $2 \%$ of the uniform field value $V / t$, several parallel plate structures were examined.

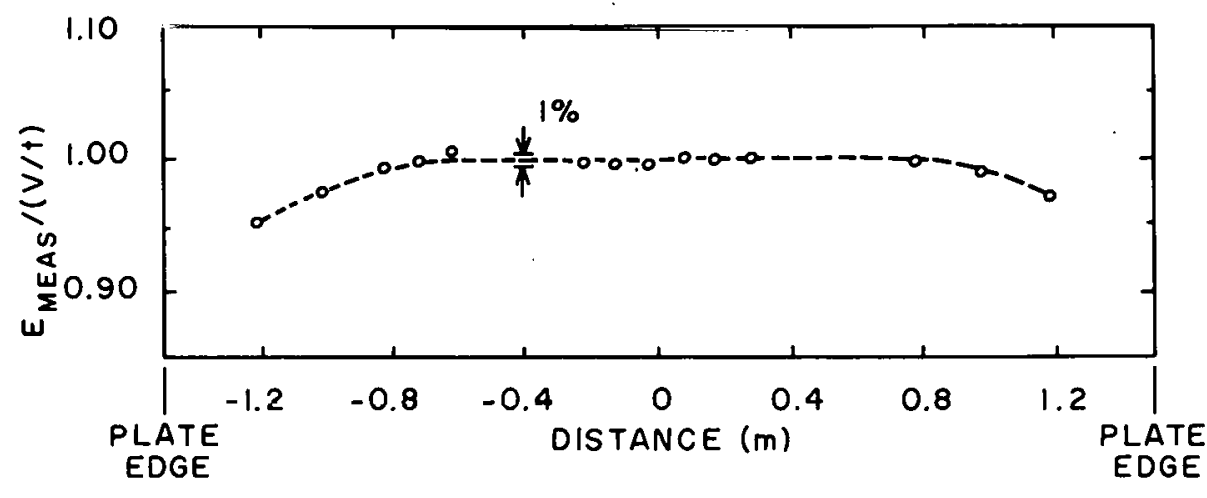

Figure 14. The E-field along the bottom plate is within $1 \%$ of the uniform value, $V / t$, over an exlended region. The lack of symmctry at the left is largely due to the "window" in the band structure. 
Facilitating this study was the development of a miniature E-field probe. Because of its small size, it could be used to map the field profiles over large regions without significant perturbation of the surface charge distributions. Some details of the probe and its operation are given in Appendix $D$.

\section{Parallel-Plate Calibration Structures}

An initial design consideration is the minimum plate spacing which can be used without introducing serious feedback effects because of surface charge perturbations. The data of Figure 11 indicate that the spacing must be at least $0.75 \mathrm{~m}$ to avoid measurable effects. Because the data were obtained with a Type B meter ( $19 \mathrm{~cm}$ vertical dimension), a $0.75 \mathrm{~m}$ spacing will also be adequate for the Type A meters which are smaller. Using this spacing, a number of parallel plate systems were examined with the miniature field probe. It was found that a parallel plate system $1 \mathrm{~m} \times 1 \mathrm{~m} \times 0.75 \mathrm{~m}$ with four equally-spaced guard rings contained a central $20 \times 20 \times 20 \mathrm{~cm}^{3}$ volume in which the E-field value remained constant to within $\sim 0.6 \%$. The magnitude of the vertical E-field at the center of the structure was about $1 \%$ less than the uniform value, $V / t$. The balanced voltage arrangement and adequate current limiting resistors were again employed; the bottom plate was $\sim 0.8 \mathrm{~m}$ above the floor.

The system was tested as a calibration facility by comparing measured E-field values at the center of this structure with values measured in the larger $3 \mathrm{~m} \times 3 \mathrm{~m} \times 0.75 \mathrm{~m}$ apparatus for the same $\mathrm{V} / \mathrm{t}$. Both commercial meters were used for the comparison. The Type A meter readings were $\sim i .0-1.7 \%$ less in the smaller guarded structure. The Type B meter readings appeared to be $\sim 0.0-0.6 \% 1$ ess in the guarded structure. Thus, both meters read values that are within $2 \%$ of the uniform field value $\mathrm{V} / \mathrm{t}$. The lowered readings in the smaller guarded structure are compatible with the fact that the field at the center is less than $V / t$ by $\sim 1 \%$. 
A second calibration system without guard rings and having greater accuracy was characterized from modeling experiments. Examination of a $1 \mathrm{~m} \times 1 \mathrm{~m} \times 0.5 \mathrm{~m}$ structure with the miniature probe revealed a central $20 \times 20 \times 20 \mathrm{~cm}^{3}$ volume in which the field value did not differ from $V / t$ by more than $\sim 0.3 \%$. This result was not affected when two metal grounded "walls" (metal fences) were brought to within $0.25 \mathrm{~m}$ of the plates on opposite sides of the structure. If the model is scaled upward in size to $0.75 \mathrm{~m}$ spaçing to el iminate measurable feedback effects, the above results indicate that a structure $1.5 \mathrm{~m} \times 1.5 \mathrm{~m} \times 0.75 \mathrm{~m}$ will provide a usable calibration field which is well within $1 \%$ of the uniform field $V / t$.

The perturbations due to nearby walls (floor) will not be significant if they are half the plate spacing or more away from the plates.

Briefly summarizing the characteristics of the calibration facilities described above, we have:

$\begin{array}{lcccc}\text { Applied Voltage } & \text { Dimensions } & \text { Guard Rings } & \text { Error } & \begin{array}{c}\text { Wall/Floor } \\ \text { Distance }\end{array} \\ \text { Balanced-to-ground } & 1 \times 1 \times 0.75 \mathrm{~m} & 4 & <2 \% & 0.4 \mathrm{~m} \\ \text { Balanced-to-ground } & 1.5 \times 1.5 \times 0.75 \mathrm{~m} & 0 & <1 \% & >0.4 \mathrm{~m}\end{array}$

\section{Current Injection Calibration}

The field meters being studied can be considered current measuring devices which in use are capacitively coupled to a high voltage source and to ground. (As indicated in Section II.A, the Type A field meter measures the charge on the sensing electrodes; however, since this is accomplished by integrating the current, the concept of "current injection" is appropriate for both types). The two functions, current measurement and capacitance coupling, are independent parameters and may be treated separately. The capacitance coupling is a function 
of the total geometry and is influenced, of course, by the proximity of other objects (grounded, floating, or connected to the high voltage source) as well as by the meter's location with respect to the high-voltage lines and the ground plane and by the geometry of the meter itself.

The current injection "calibration" procedure, hereafter referred to as a "current calibration," provides a convenient method of monitoring the current measurement parameter and determining a correction factor to be applied to the meter readings in the event that it changes with time.

The Type B meter is provided with an input jack for a current calibration. Insertion of a plug into the jack connects the two sensing electrode structures together to provide a shield. A current injection circuit, such as shown in Figure 15, which permits this shield to be grounded during the calibration eliminates the problem of interference from stray fields normally encountered in a laboratory, e.g., from fluorescent lights, unshielded power raceways, extension cords, etc.

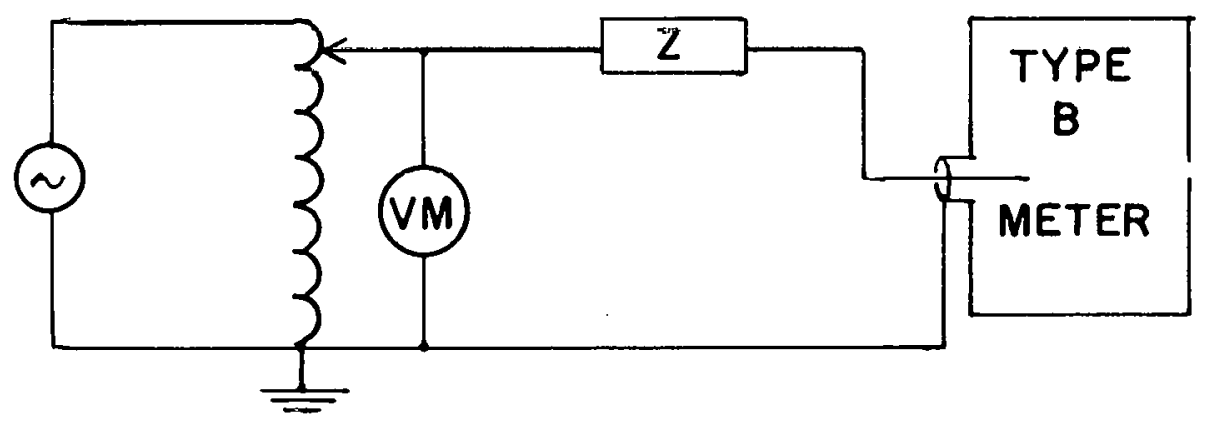

Figure 15

VM is a precision voltmeter and $Z$ a known impedance, large compared with the input impedance of the field meter.

The manufacturer's instruction manual for the Type $B$ meterigives $193 \times 10^{-12}$ amperes/(volt/meter) as the design value for the current sensitivity. Choice of 
the series impedance, of course, determines the voltage required to obtain full scale deflection on a given range. The input impedance of the Type $B$ meter (of the order of $1000 \Omega$ for the ranges of interest) may be neglected if the series impedance selected is $10^{6}$ ohms or larger.

Current calibration of the Type A meter is accomplished by attaching leads to the sensing plates. During calibration the meter is in its normal operating condition and thus responds to stray ambient fields. The interference of stray fields proved to be least when the current limiting impedances were introduced in a balanced configuration as shown in Figure 16.

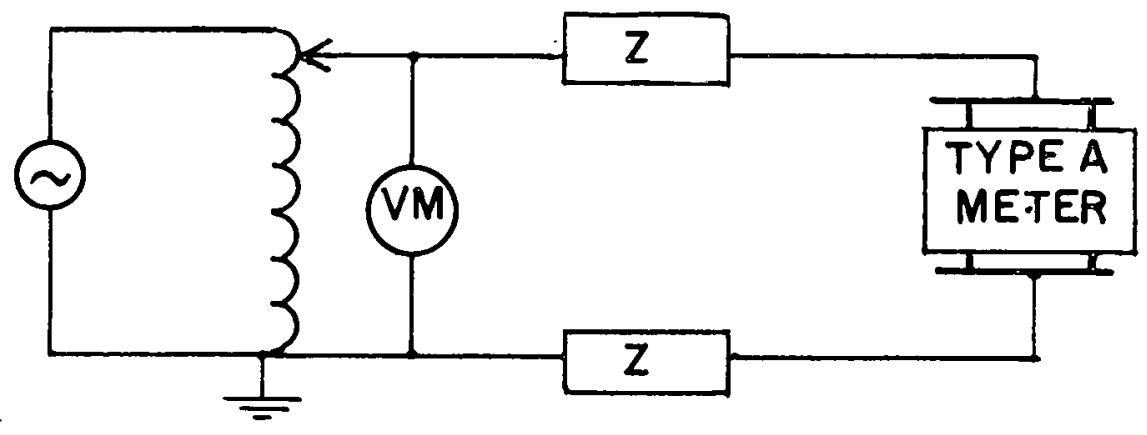

Figure 16

Use of a voltage supply which was balanced to ground did not further reduce the influence of stray fields.

An indication of the magnitude of interfering ambient fields may be obtained by changing the phase relationship between the calibrating and interfering signals. This may be done by interchanging the lcad connections to the sensing plates of the meter being calibrated or by reversing connections to the power supply. If the calibrating voltage required for a given meter reading is the same for the two arrangements, the interfering signal is negligibly small. If a small difference 
exists, the average of the two voltage values is that which would be required in the absence of interference.

The manufacturer's instruction manual for the Type $A$ meter implies a value of $100.8 \times 10^{-12}$ ampere-meter/volt for the current sensitivity. Choice of impedance values and supply voltages are dictated by convenience except for the limitations imposed by the input impedance of the meter--of the order of $10^{4}$ ohms.

Examples of suitable impedance values for current calibrations of the meter ranges of greatest interest are given in the following table:

\section{Type A Field Meter}

\begin{tabular}{|c|c|c|}
\hline$\frac{\text { Range }}{\mathrm{kV} / \mathrm{m}}$ & (inpedance $^{\text {In each arm) }}$ & $\begin{array}{l}\text { Approximate Voltage } \\
\text { Full-Scale Deflection } \\
\text { (volts) }\end{array}$ \\
\hline 5 & $\begin{array}{r}.5 \times 10^{6} \\
5 \times 10^{6}\end{array}$ & $\frac{1.5}{5}$ \\
\hline 10 & $\begin{array}{r}1.5 \times 10^{6} \\
5 \times 10^{6}\end{array}$ & $\begin{array}{r}3 \\
10\end{array}$ \\
\hline 25 & $\begin{array}{r}1.5 \times 10^{6} \\
5 \times 10^{6}\end{array}$ & 25.5 \\
\hline
\end{tabular}

\section{Type B Field Meter}

$\begin{array}{ccc}\text { Range } & \frac{\text { Impedance }}{\text { k.V/m }} & \begin{array}{c}\text { Approximate Voltage } \\ \text { Full Scale Deflection }\end{array} \\ 3 & 10^{6} & 0.6 \\ & 10^{7} & 6 \\ 10 & 10^{6} & 1.9 \\ & 10^{7} & 19 \\ 30 & 10^{6} & 6 \\ & 10^{7} & 60\end{array}$

* As indicated in the text, resistors as series impedance elements in general are preferred. However, if the source voltage has negligible harmonic distortion capacitors may be used instead. 
Since the current calibration is intended only to monitor the stability of the current measuring circuit by periodic calibration, the values of the impedances need not be known with high accuracy. It is essential only that they be stable. Thus, verification of the stability of the resistors used may be accomplished by dc measurements, and the stability of the capacitors used may be determired by measurements at $1 \mathrm{kHz}--a l$ though in each case the values thus measured may differ from those which exist at the frequency of use, $60 \mathrm{~Hz}$.

Special shielding of the impedances is not required unless the values selected differ by orders of magnitude from those given in the tables.

Care must be taken to insure that the calibrating voltage waveform closely approximates a sine wave. As discussed in Section II.B.l.f, the percentage error in the calibration resulting from a non-sinusoidal voltage waveform under certain circumstances may be as large as the percent harmonic distortion existing in that voltage. In general, iron-core transformers, being non-linear devices, are sources of waveform distortions. The design of the adjustable voltage supply for use in current injection calibrations should be examined carefully and checked experimentally, if feasible, to assure its adequacy with respect to the requirement of low distortion.

In view of these waveform considerations there is some advantage in using resistors rather than capacitors as the series impedance elements in the circuits of Figures 15 and 16 . Since the admittance of a capacitor increases with increasing frequency, the error contribution of the harmonic components of the source waveform is larger with a capacitor than with a resistor. 


\section{Magnetic Field Measurements}

An accessory is available from the manufacturer of the Type B electric field meter which extends its use to the measurement of $60 \mathrm{~Hz}$ magnetic fields. An adapter is provided to convert the field meter to a voltmeter mode. The probe is an electrostatically shielded multiturn loop.

\section{A. Instrument Performance}

A magnetic field measuring instrument of the type described above was used to measure the $60 \mathrm{~Hz}$ magnetic field (B-field) at the center of a calibrated 1.5 meter Helmholtz coil. Prior to the ac measurements, a dc field was generated and found to be uniform to within $0.2 \%$ over the volume of the field probe. This was determined by nearly nulling the earth's magnetic field and measuring the field variations over the volume of the probe to be calibrated with a sensitive magnetometer.

The errors in the measurement of fields over the range of $7 \times 10^{-7}$ to $1.9 \times 10^{-4}$ tesla (0.007 to 1.9 gauss) using this particular instrument were as indicated in Figure 17.

The influence of ambient electric fields on the operation of the magnetic field probe was examined in a field near $5.3 \mathrm{kV} / \mathrm{m}$ using the parallel plate structure described in Appendix B.i. A Helmholtz coil, with its axis parallel to the E-field direction, was placed between the parallel plates. Measurements of the background $60 \mathrm{~Hz}$ B-fields near the center of the parallel plate Helmholtz coil system with and without the E-field showed an increase in the B-field reading of about 0.15 milligauss in the presence of the E-field. This result was independent of whether the Type B E-field meter and adapter were in or out of the E-field, and independent of the orientation of the probe relative to the E-field. The small perturbation of the B-field reading due to the E-field could also be seen on the more sensitive ranges of the meter $\left(\leq 10^{-6}\right.$ tesla $(0.01$ gauss $)$ full scale $)$ when a small B-field was applied with the Helmholtz coils; however, it could 
not be resolved on the higher meter ranges $\left(\geq 10^{-5}\right.$ tesla ( 0.100 gauss) full scale) when the magnitude of the B-field was increased. These results indicate that there was no serious inadequacy in the electrostatic shielding.

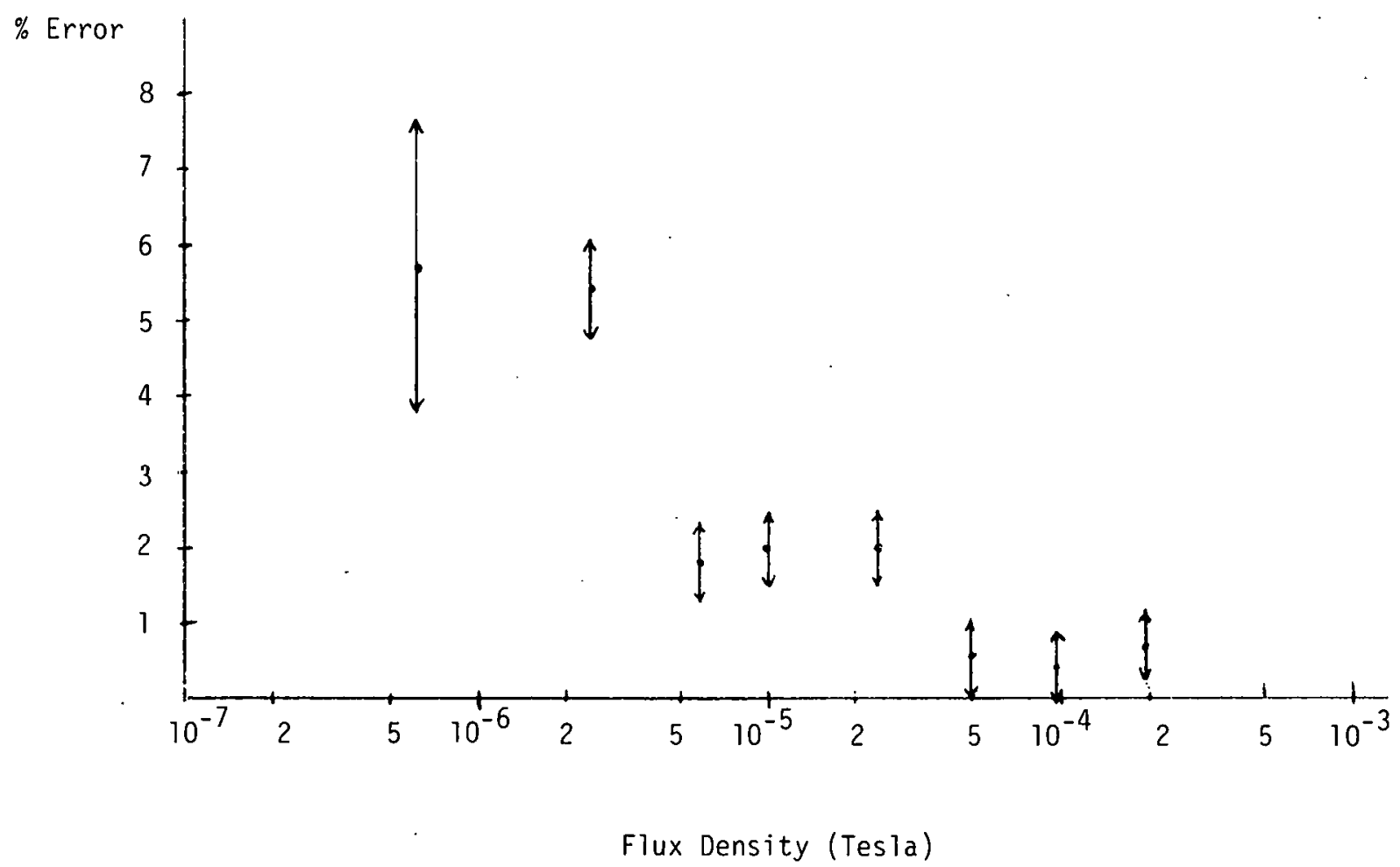

Figure 17. Magnetic field measurement errors using a Type B electric field meter, magnetic field probe and adapter. The uncertainties indicated in the errors result from measurement uncertainties in the $60 \mathrm{~Hz}$ calibrating field.

Because the probe signal is dependent on the magnetic field frequency (Faraday's Law), the discussion pertaining to the response of the Type B electric field meter to field harmonics (Section II.B.l.f) is again applicable when magnetic fields are measured.

B. Calibration of Magnetic Field Meters

Calibration of a magnetic field probe is typically done by introducing the probe into a nearly uniform magnetic field of known magnitude and direction. Helmholtz coils have frequently been employed to generate such fields, but the 
more simply constructed single loop (of many turns) with rectangular geometry has also been used. The simplicity in construction is at the expense of reduced field uniformity, but sufficient accuracy is readily obtained. The z-component of the magnetic field produced by a rectangular loop of dimensions $2 \mathrm{a} \times 2 \mathrm{~b}$ is given by the expression [14]

$$
\dot{B}_{z}=\frac{\mu_{0}}{4 \pi} \text { IN } \sum_{\alpha=1}^{4} \frac{(-1)^{\alpha} d_{\alpha}}{r_{\alpha}\left[r_{\alpha}+(-1)^{\alpha+1} c_{\alpha}\right]}-\frac{c_{\alpha}}{r_{\alpha}\left(r_{\alpha}+d_{\alpha}\right)}
$$

where

$$
\begin{array}{ll}
N=\text { number of turns } \\
c_{1}=-c_{4}=a+x & r_{1}=\sqrt{(a+x)^{2}+(b+y)^{2}+z^{2}} \\
c_{2}=-c_{3}=a-x & r_{2}=\sqrt{(a-x)^{2}+(b+y)^{2}+z^{2}} \\
d_{1}=d_{2}=b+y & r_{3}=\sqrt{(a-x)^{2}+(b-y)^{2}+z^{2}} \\
d_{3}=d_{4}=y-b & r_{4}=\sqrt{(a+x)^{2}+(b-y)^{2}+z^{2}} .
\end{array}
$$

$I$ is the current, $\mu_{0}$ is the magnetic permeability of air and the coordinates $x, y$, and $z$ are shown in Figure 18. The conductors in the current loop are assumed to be of small cross section. It is noted for purposes of reference that 
$B_{z}(0,0,0)=\mu_{0}$ I $N \sqrt{2} / \pi a$ for a square loop of side dimension 2a. Equation 7 has been used to calculate the field values at and near the center of a rectangular loop of dimensions $5 \mathrm{~m} \times 0.53 \mathrm{~m}$. The percent departure from the central magnetic field value at nearby points in the plane of the loop and $3 \mathrm{~cm}$ above the plane of the loop are plotted in Figure 19. A similar plot is shown in Figure 20 for a square loop having dimensions $1 \mathrm{~m} \times 1 \mathrm{~m}$. Also shown in Figures 19 and 20 is an approximate outline (actual size) of the magnetic field probe. Examination of the two figures indicates that the square loop, with a smaller cross sectional area, provides a more uniform field.

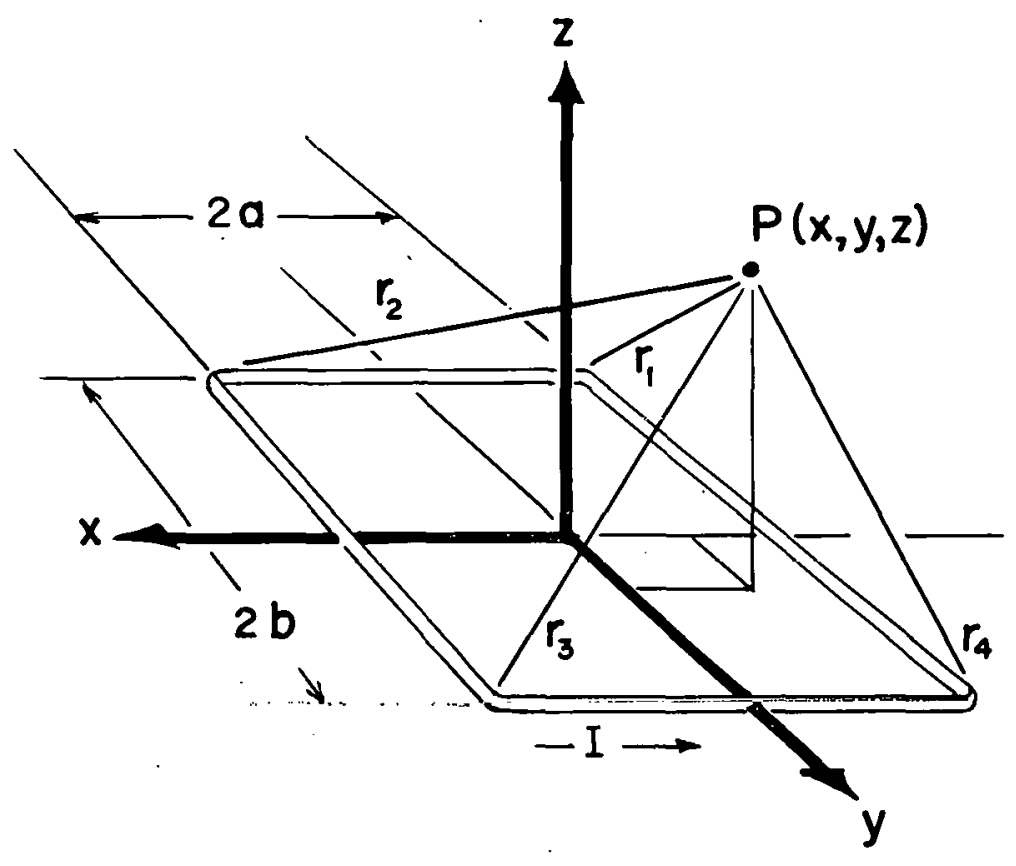

Figure 18. Coordinate system for current loop generating magnetic field $B_{z}$.

It should be noted that an error in centering a $12 \mathrm{~cm}$ diameter sensing coil of $2 \mathrm{~cm}$ in any of the three orthogonal directions introduces an error of less than $1 \%$ in the calibration. Any uncertainty in the effective loop dimensions is reflected as the same percent error in the magnetic field produced. 


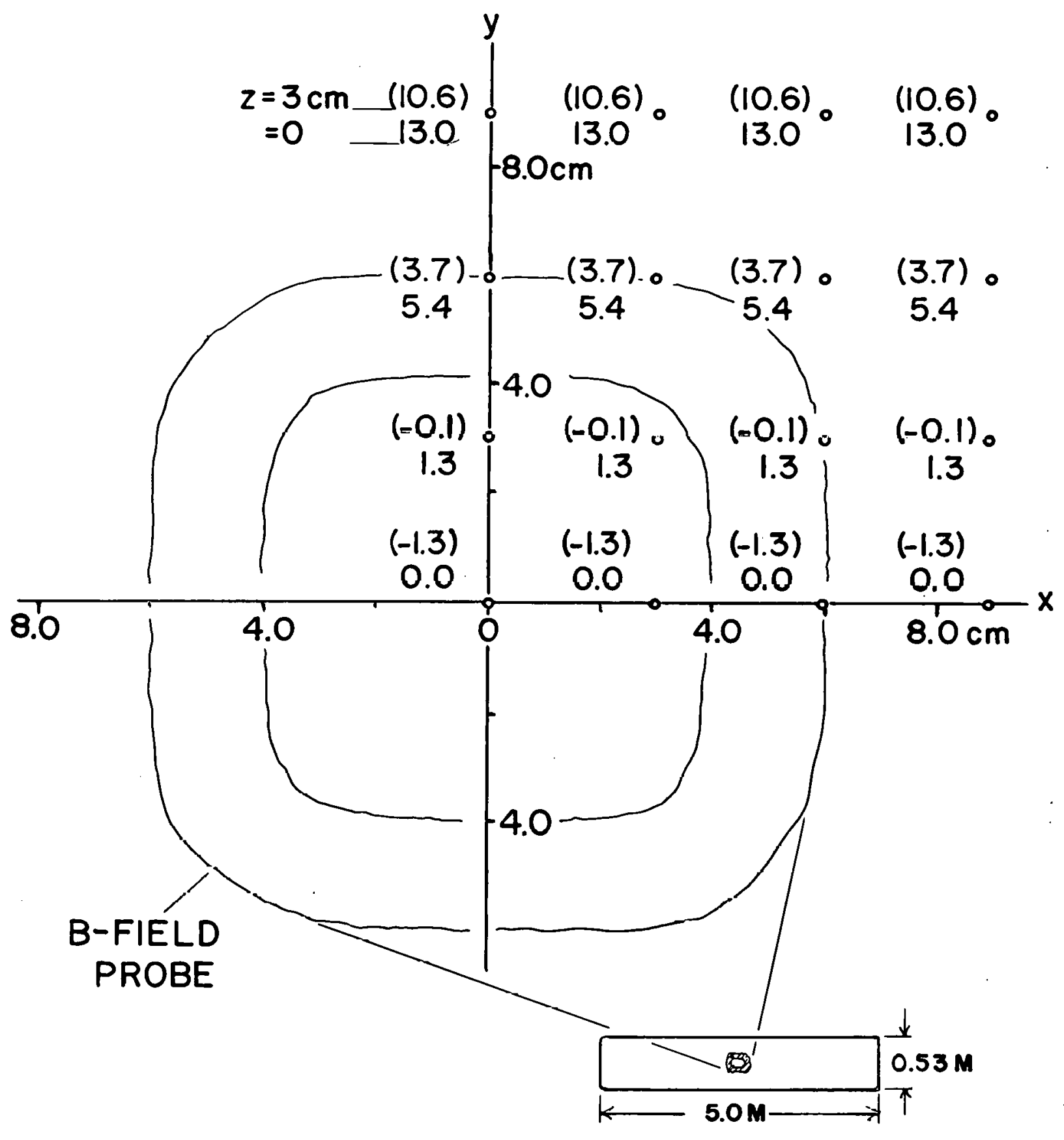

Figure 19. Percentage departure of $B_{z}$ from $B_{z}(0,0,0)$ for positions in the plane of a rectangular current loop $5 \mathrm{~m} \times 0.53 \mathrm{~m}$ and $3 \mathrm{~cm}$ above or below the plane (brackets). 


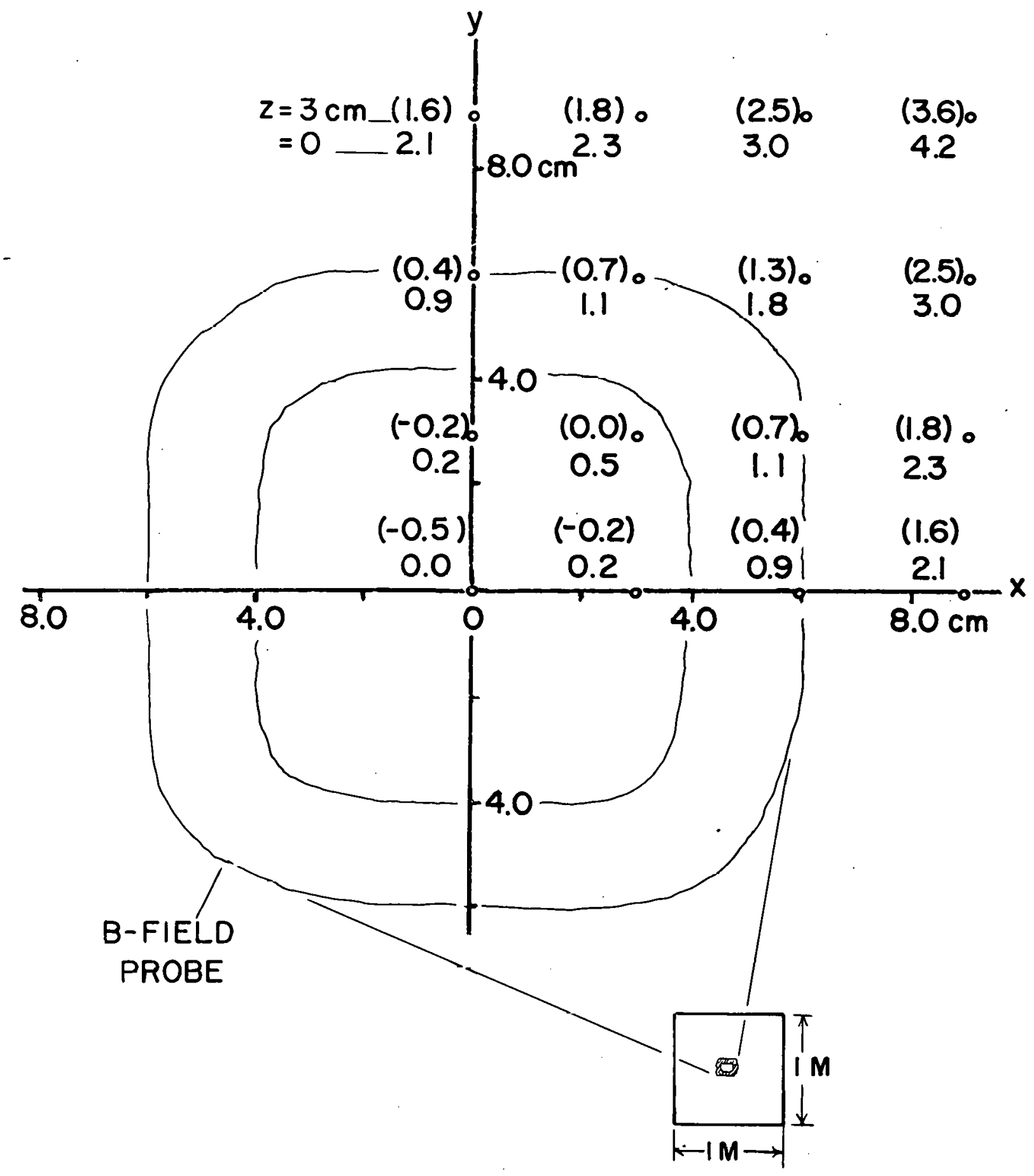

Figure 20. Percentage departure of $B_{z}$ from $B_{2}(0,0,0)$ for positions in the plane of a square current loop $1 \mathrm{~m} \times 1 \mathrm{~m}$ and $3 \mathrm{~cm}$ above or below the plane (brackels). 


\section{Appendix A}

Current Induced in Conducting Sphere by

Quasistatic Electric Field From Point Charge

The electric potential, $U$, at points exterior to an uncharged spherical conductor of radius $a$ in the vicinity of a point charge $q(\omega t)$ can be shown to be, using the method of images [15]

$$
U(r, \theta, \phi, \omega t)=\frac{1}{4 \pi \varepsilon_{0}}\left[\frac{q}{r_{1}}+\frac{q^{\prime}}{r_{2}}-\frac{q^{\prime}}{r}\right]
$$

where

$$
\begin{gathered}
r_{1}=\sqrt{r^{2}+d^{2}-2 r d \cos \theta} \\
r_{2}=\sqrt{r^{2}+b^{2}-2 r b \cos \theta} \\
4 \quad q \sin \omega t=q(\omega t) \\
q^{\prime}==(a / d) q \\
b=a^{2} / d
\end{gathered}
$$

and $d$ is the distance between the point charge and the center of the sphere; $\mathrm{q}(\omega \mathrm{t})$ is assumed to have a quasistatic time dependence. The geometry of the prohlem is shown in Figure Al. 


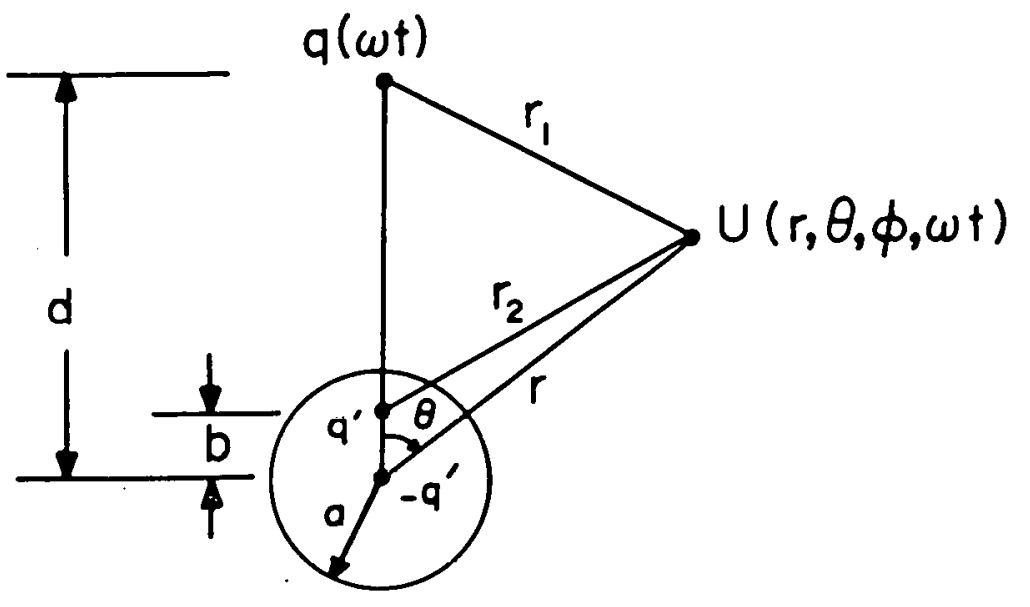

Figure Al

To determine the induced current, the surface charge density $\sigma$ is first found from the relation

$$
\sigma=-\left.\varepsilon_{0} \frac{\partial U}{\partial r}\right|_{r=a} \text {. }
$$

The total induced charge $Q$ on the hemisphere facing the point charge can then be calculated using Equation $A 2$ and differentiation with respect to time will yield the current. Details of the algebra are briefly presented below.

Differentiating Equation $A$ with respect to $r$ and setting $r=a$ yields

$$
\left.\frac{\partial U}{\partial r}\right|_{r=a}=-\frac{a}{4 \pi \varepsilon_{0}} \frac{1}{a d}\left[\frac{1-(a / d)^{2}}{\left(1+(a / d)^{2}-2(a / d) \cos \theta\right)^{3 / 2}}-1\right] .
$$

It is convenient to rewrite Equation $A 3$ as

$$
\left.\frac{\partial U}{\partial r}\right|_{r=a}=\frac{E}{s}\left[\frac{1-s^{2}}{\left(1+s^{2}-2 s \cos \theta\right)^{3 / 2}}-1\right]
$$


where

$$
E=q / 4 \pi \varepsilon_{0} d^{2}
$$

and $s=a / d$. The surface charge density is, therefore,

$$
\sigma=-\varepsilon_{0} \frac{E}{s}\left[\frac{\left(1-s^{2}\right)}{\left(1+s^{2}-2 s \cos \theta\right)^{3 / 2}}-1\right] .
$$

The radical can be expanded in powers of $\left(s^{2}-2 s \cos \theta\right)$ which is less than one; for purposes of this derivation only terms of order $s^{4}$ or less need be retained. Performing the term by term integration of the expanded $\sigma$ over the surface of the top hemisphere leads to

$$
\begin{aligned}
Q & =\int_{0}^{2 \pi} \int_{0}^{\pi / 2} 0 a^{2} \sin \theta d \theta d \phi \\
& =3 \pi \varepsilon_{0} E a^{2}\left[1-(7 / 12) s^{2}+\frac{11}{24} s^{4}+\ldots\right] .
\end{aligned}
$$

The current which flows between isolated hemispheres is given by

$$
I=\frac{d Q}{d t}=3 \pi \varepsilon_{0} \omega E a^{2}\left[1-\frac{7}{12}\left(\frac{a}{d}\right)^{2}+\frac{11}{24}\left(\frac{a}{d}\right)^{4} \ldots\right] .
$$

It should be noted that Equation A6 has been derived for the case when the hemispheres are aligned with the E-field direction. 


\section{Appendix B \\ The Electric Field Between Parallel Plates \\ --One Plate Grounded}

While conceptually the calibration procedure of introducing a field meter, at the end of an insulating rod, between electrically energized parallel plates seems straightforward, the following effects must be considered in order to establish a well defined E-field: (i) perturbation of the surface charge density on the plates, $\sigma$, by the field meter, and ( $i i)$ perturbation of $\sigma$ by nearby ground planes. These effects are now considered in some detail both theoretically and experimentally. The effectiveness of guard rings in reducing the influence of fringing fields and of nearby ground planes is also explored experimentally (iii) and theoretically (iv).

(i) Perturbation of Surface Charge Density By Field Meter

Theory. Ideally the parallel plates should generate a uniform field of sufficient extent in space that introduction of the field meter introduces negligible perturbation of the field or charge distribution at the plates. Estimation of surface charge density perturbations has been made in the following way. First a conducting sphere or ellipsoid of appropriate dimensions is chosen to represent the field meter (animals in biological experiments have similarly been represented). It is next considered to be introduced into a uniform field, and perturbations of the uniform field in a plane, where one of the metal electrodes for producing the field will eventually be placed, are calculated from known solutions to the problem (see, for example, Battelle Northwest Interim Progress Report to ERDA, "Biological Effects of High Strength Electric Fields on Small Laboratory Animals," September 1976. A draft dated April 1976 on E-field measurements prepared by CIGRE Working Group 36-01 
also considers the problem in a similar way). It is then assumed that the perturbation of the surface charge density, $\sigma$, on a conducting surface located in the plane will be nearly the same percentage as that of the field perturbation because of the direct proportionality between the surface charge density and the electric field at the surface.

The perturbed electric field $E$ for the case of a sphere is given in spherical coordinates by $[16]$

$$
\frac{E}{E_{0}}=\left[\left(1+\frac{2 a^{3}{ }^{2}}{r^{3}}\right)^{2} \cos ^{2} \theta+\left(1-\frac{a^{3}}{r^{3}}\right)^{2} \sin ^{2} \theta\right]^{1 / 2}
$$

where $a$ is the radius of the metal sphere, $r$ is the radial distance from the sphere's center and $\theta$ is the angle the position vector $\vec{r}$ makes with $\vec{E}_{0}$. Equation $B 1$ indicates that for a sphere of radius $10 \mathrm{~cm}, E / E_{0}$ has come within $1 \%$ of the uniform value when $r=60 \mathrm{~cm}$ and $50 \mathrm{~cm}$ for $\theta=0$ and 90 degrees respectively.

Experiment. To test this approximate theory experimentally, a parallel plate apparatus was constructed which permitted measurement of the surface charge density on one of the plates. A schematic view is shown in Figure. B1. Two metal sheets $122 \mathrm{~cm} \times 92 \mathrm{~cm}$ are separated by a distance of $38 \mathrm{~cm}$. The top plate is connected to a $60 \mathrm{~Hz}$ high voltage source and the bottom plate, with the exception of ten isolated segments, is connected to ground. The E-field induced current, I, in the isolated segments is measured with an operational amplifier and rms reading digital voltmeter. A switching system grounds all the segments except one which is being monitored. After the current has been measured, the rms value of the electric field averaged over the area, $A$, of the segment is determined from the relation

$$
E=\frac{I}{120 \pi \varepsilon_{0} A} .
$$




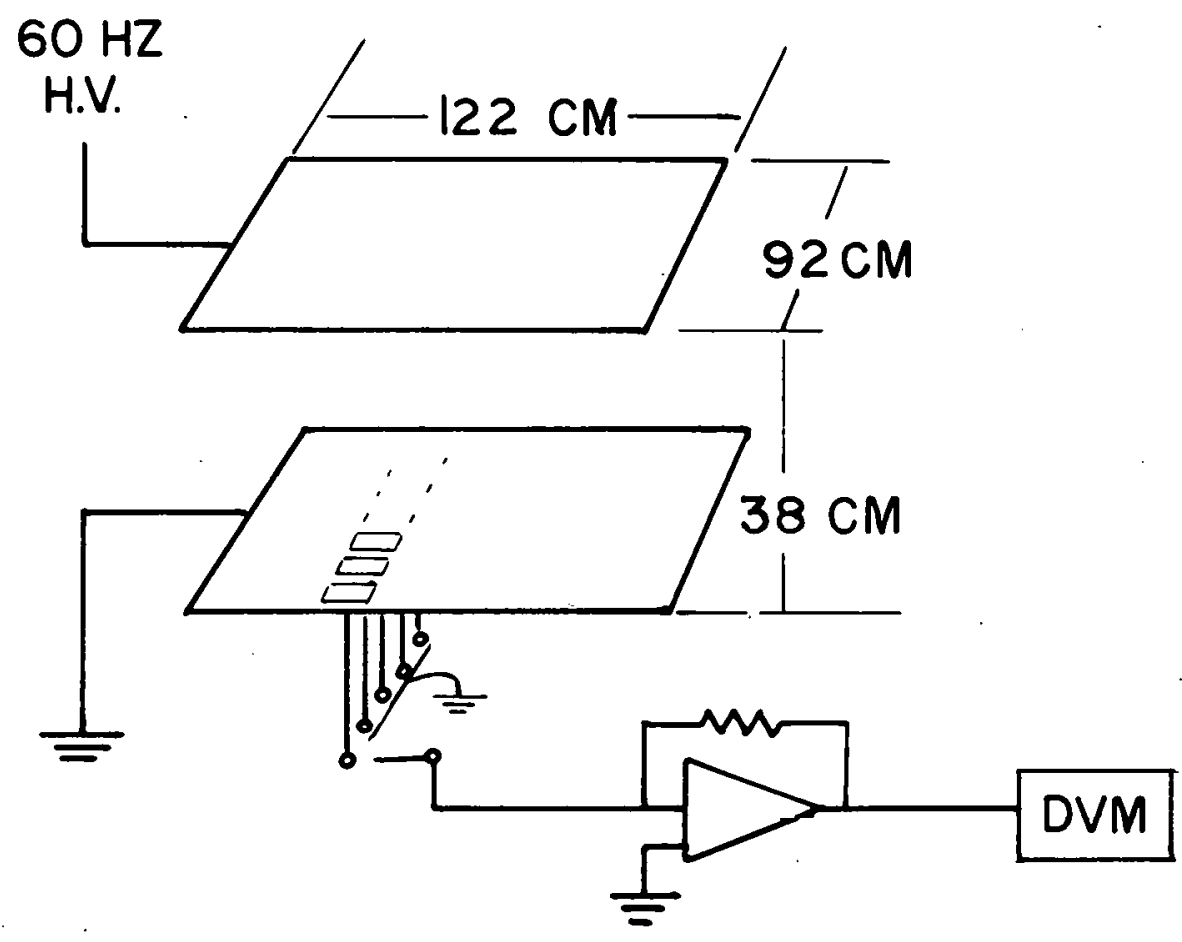

Figure Bl. Schematic view of apparatus for perturbation measurements.

Here $\varepsilon_{0}$ is the permittivity of free space. Equation B2 is obtained by applying Gauss' Law to the isolated segment and differentiating with respect to time.

Figure B2 shows measured values of the electric field as a function of distance from the edge of the bottom plate with no objects placed between the plates. Each value has been normalized by the "uniform field," $E_{0}$, measured at the center of the plate. The experimental value, $E_{0}$, is within $\pm 0.4 \%$ of the theoretical value $V / t$, where $V$ is the applied rms voltage and $t$ is the plate spacing. The observed discrepancy is comparable to the largest experimental uncertainty which is the value of the center segment area $A$.

Also shown in Figure B2 is the theoretical normalized field dependence as a function of position [11]. Good agreement is seen between experiment and theory for distances up to about $25 \mathrm{~cm}$ from the plate center. For positions closer to the 
edge, the experimental data are consistently lower in value. Qualitatively, much of this is explained by the absence of any surrounding ground planes in the theoretical treatment of the problem (see Appendix B.ii, below).

Measurements of surface charge densities on the bottom plate of a parallel plate system before and after the introduction of isolated conducting spheres were

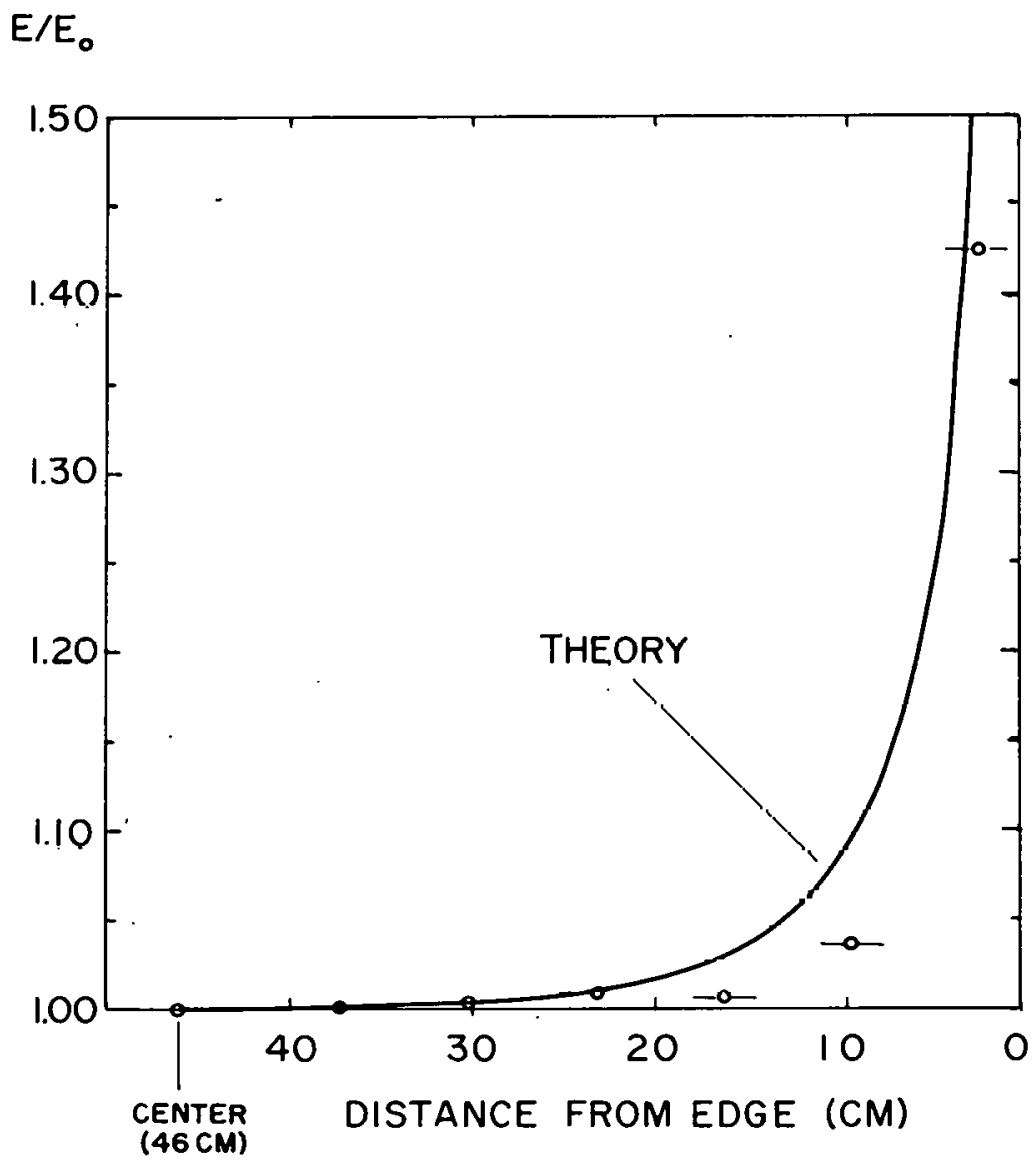

Figure B2. Experimental and theoretical field distribution along bottom plate.

subsequently made. The perturbation of the surface charge density directly below the sphere was compared with the predictions of Equation $\mathrm{Bl}$ for $\theta=0$

$$
\frac{E}{E_{0}}=\frac{\sigma}{\sigma_{0}}=\left(1+2 \frac{a^{3}}{r^{3}}\right)
$$


The results are tabulated below.

$$
\text { sphere radius (a) distance } r \quad\left(\sigma / \sigma_{0}\right)_{\text {est. }}=1+2\left(\frac{a}{r}\right)^{3} \quad\left(\sigma / \sigma_{0}\right)_{\text {meas. }}
$$
(i.)
$3.83 \mathrm{~cm}$
$19.3 \mathrm{~cm}$
1.0156
1.030
(ii)
2.37
18.5
1.0042
1.010
(iii)
2.37
20.0
1.0033
1.006

The measured $\sigma / \sigma_{0}$ values are believed to be accurate to within $\pm 0.1 \%$. It can be seen that for the chosen $a / r$ values, the estimated perturbations are in error by a factor of about two. The error can be expected to increase for 1 arger $a / r$ ratios because introduction of a conducting surface in the E-field will enhance the field at an increasing rate as qualitatively shown in Figure B3.

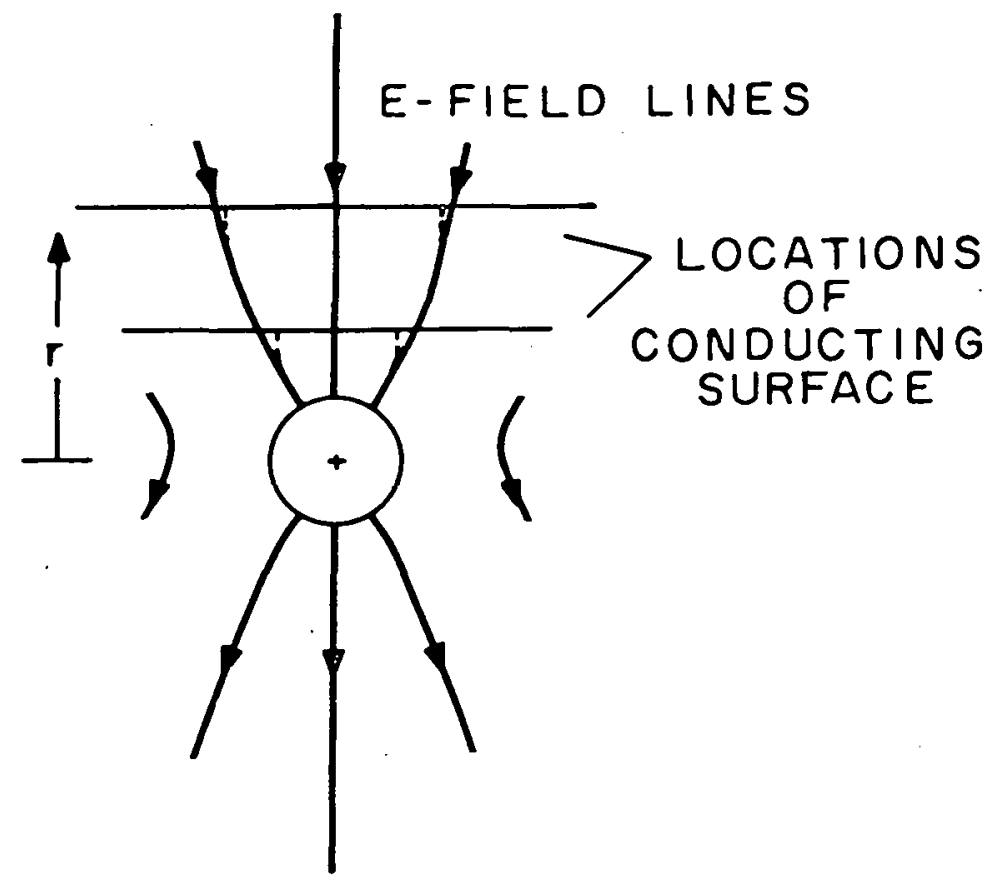

Figure B3

Using scaled-down versions of the two types of electric field meters, the field perturbation at the center of the bottom plate was measured as a function of 
height, $H$, above the plate. The results for metallic boxes approximately $1 / 2$ and $1 / 3$ the size of the Type $B$ meter and approximately $1 / 3$ the size of the Type $A$ meter are shown in Figure $B 4$. The perturbed field, $E_{p}$, has been divided by the experimental value of $E_{0}$. The height $H$ is measured from the box center to the plate surface. It can be seen that in all cases, the field perturbation at the center of the bottom plate exceeds $+1 \%$ when the metallic boxes are midway $(19 \mathrm{~cm})$ between the plates. The same effect occurs at the top plate.

Perturbation measurements were also made at other points along the bottom plate as the height of the simulated meters was varied. A metallic hox approximately $1 / 2$ the size of the Type B meter was used to obtain the data presented in Figure B5. The data show that the influence of the box becomes negligible as positions, closer to the plate edge are monitored.

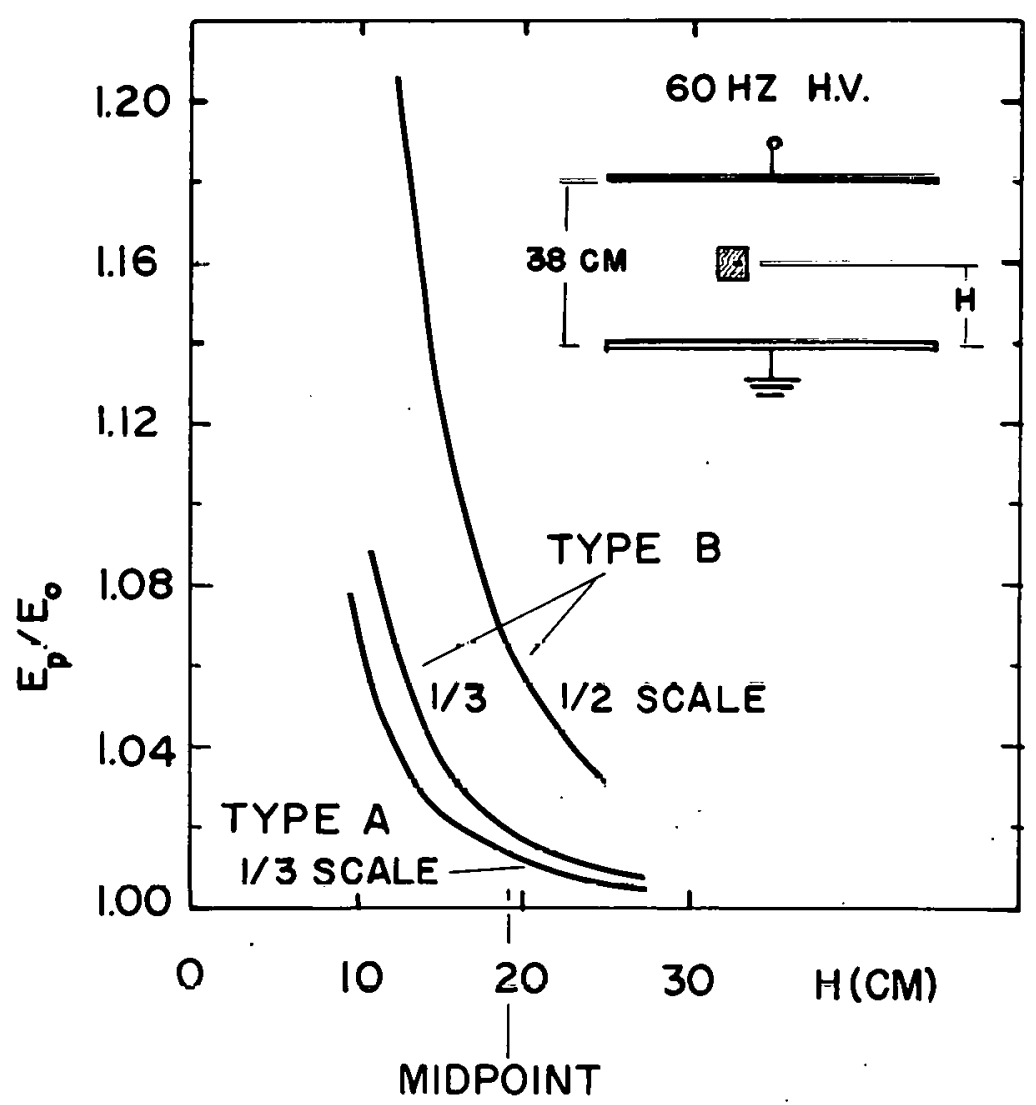

Figure B4. Field perturbations at center of bottom plate produced by modeled field meters. 


\section{Discussion}

Concern regarding the magnitude and distribution of the field perturbation at the plate surface arises because of the "feedback" effect on the E-field at the location of the field meter which is to be calibrated. A perturbed field at the plate surface means that the surface charge distribution $\sigma\left(=E_{p} / E_{0}\right)$ has been changed. If this modified $\sigma$ is hypothetically "frozen" in place, the E-field at the position of the field meter, $\vec{r}$, (with the meter removed) can be calculated using the expression [17]

$$
E(\vec{r})=\frac{1}{4 \pi \varepsilon_{0}} \iint \frac{\sigma d a\left(\vec{r}-\vec{r}^{\prime}\right)}{\left|\vec{r} \vec{r}^{\prime}\right|^{3}} .
$$

The quantities da, $\vec{r}^{\prime}$, and $\vec{r}$ are shown in Figure B6. Calibration of a field meter at position $\vec{r}$ to an accuracy of $\mathrm{N} \%$ requires that the calculated value of $\vec{E}$ at $\vec{r}$ and the surrounding volume to be occupied by the meter not differ from $\vec{E}_{0}$ by more than $\mathrm{N} \%$ in magnitude (it is assumed here that the field direction has not significantly changed).

It is noted that an increase in $\sigma$ of $\mathrm{N} \%$ at the center of the bottom plate results in less than a N\% "feedback" effect midway between the plates. That this is the case can be seen by an examination of Figures B5 and B6 and Equation B4. The field midway between the plates is a superposition (Equation B4) of fields originating from "charges," $\sigma \mathrm{da}$. Because the perturbation of o becomes negligible near the plate edges where much of the charge is located, the summation given by Equation B4 will differ from the unperturbed case by less than N\%. This analysis was confirmed with a numerical calculation of $E$ (Equation B4) at the center of a $3 \mathrm{~m} \times 3 \mathrm{~m} \times 1 \mathrm{~m}$ spacing parallel plate system with a $2 \%$ charge density perturbation at the plate surfaces directly above and below the meter. The "feedback" effect was found to be only a few tenths of a percent. 


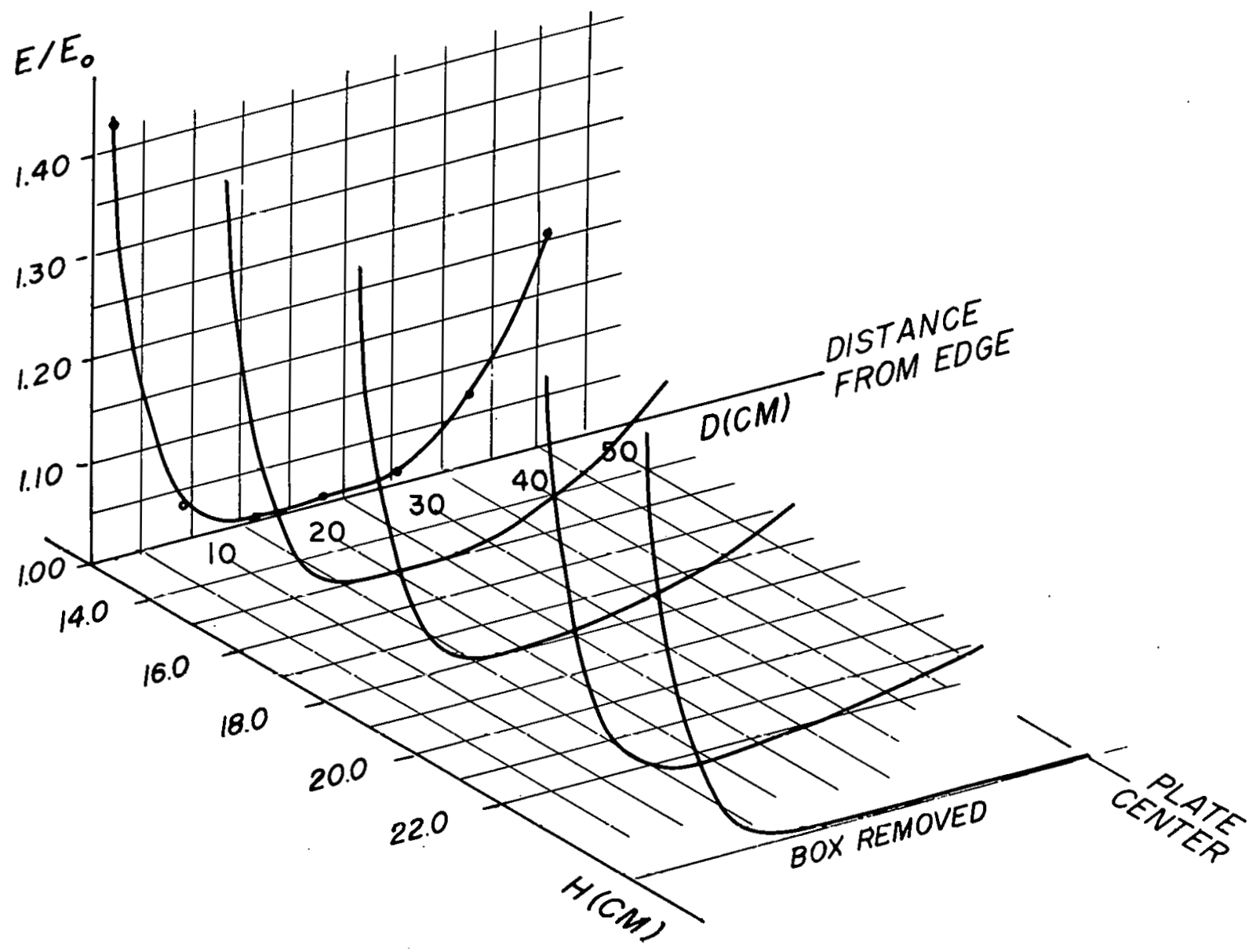

Figure B5. Distribution of perturbation at bottom plate.

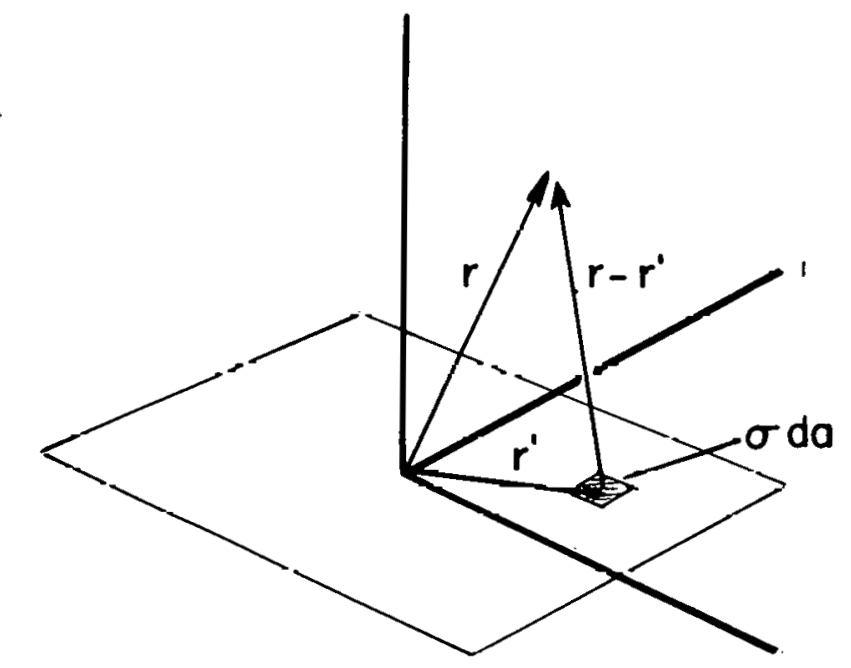

(contributions from top plate omitted for clarity) 
(ii) Perturbation of Surface Charge Density By Nearby Ground Planes

As noted in Figure $\mathrm{B} 2$, the field intensity near the bottom (grounded) plate edge was less than predicted by theory. This was attributed to the presence of nearby ground planes. To systematically study this source of perturbation, a much smaller parallel plate system, similar in design to the one described above, was constructed. Details of the smaller parallel plate structure are shown in Figure B7. Layers of aluminum foil ( $0.0025 \mathrm{~cm}$ thick) sandwich an insulating sheet of paper ( $0.005 \mathrm{~cm}$ thick) and are glued to a flat supporting plexiglass plate $(22.9 \mathrm{~cm} \times 30.5 \mathrm{~cm} \times 0.6 \mathrm{~cm})$ to form the bottom plate. The $(\mathrm{rms})$ current, I, induced in the isolated segments of the top sheet of aluminum is measured and the E-field averaged over the segment area $A$ (nominally $1 \times 8 \mathrm{~cm}^{2}$ ) is calculated using Equation B2. The bottom grounded aluminum sheet must be employed to eliminate fringing E-field lines terminating on the underside of the isolated segments. Similar shielding was used on the larger parallel plate structure.

The supporting plexiglass plate does cause some field enhancement at the edge of the plate $(\sim 10 \%)$. This perturbation, however, is essentialiy independent of the nearby ground planes and thus does not affect measurements of relative field changes as the parallel plate structure is moved closer to the ground planes.

Dielectric rods $10.2 \mathrm{~cm} \times 0.6 \mathrm{~cm}$ diameter were used to separate the plates. A difficulty encountered while using this parallel plate structure was the E-field perturbation produced by the energizing high voltage lead. This was not a significant problem with the larger parallel plates because of some self-shielding. The following measurement procedure was adopted to eliminate the problem. For a fixed distance between the ground plate (wall or floor) and parallel plates, the field distribution along the bottom plate is measured at average distances of $0.5,2.0,4.0,8.0$, and $11.4 \mathrm{~cm}$ away from the edge. Keeping the geometry fixed, a second high voltage lead is connected to the top plate and the fleld distribution 


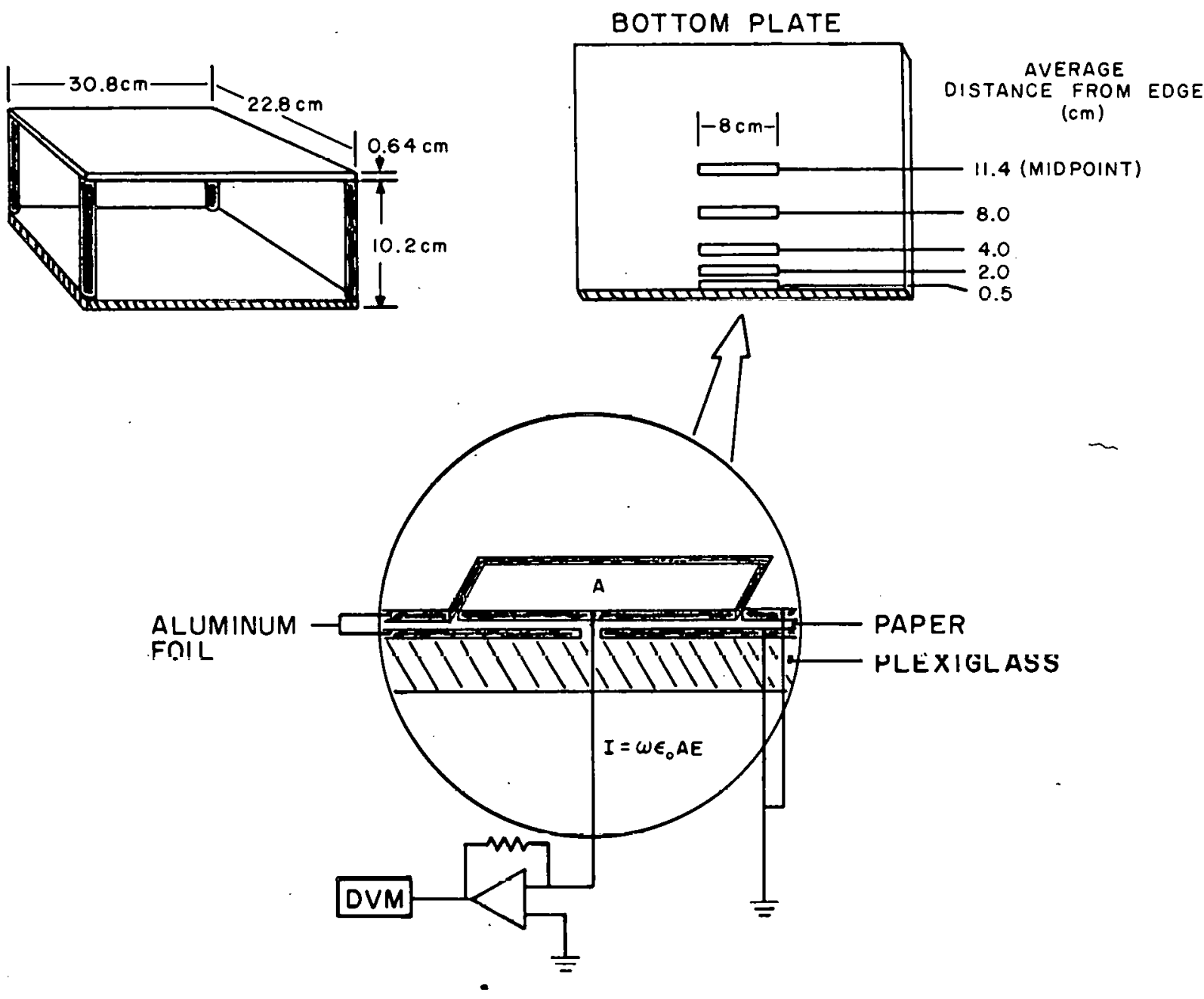

Figure B7. Construction details of smalier parallel plate system.

measurement is repeated. Because the perturbations of the two leads are additive (superposition principle), the difterence between the two sets of measurements represents the perturbations introduced by the second high voltage lead. Finally, the field distribution is measured again with only the second high voltage lead connected. The perturbations introduced by the second lead are then simply subtracted from this last set of measurements. The distance between the parallel plates and ground plane is changed and the procedure is repeated. 

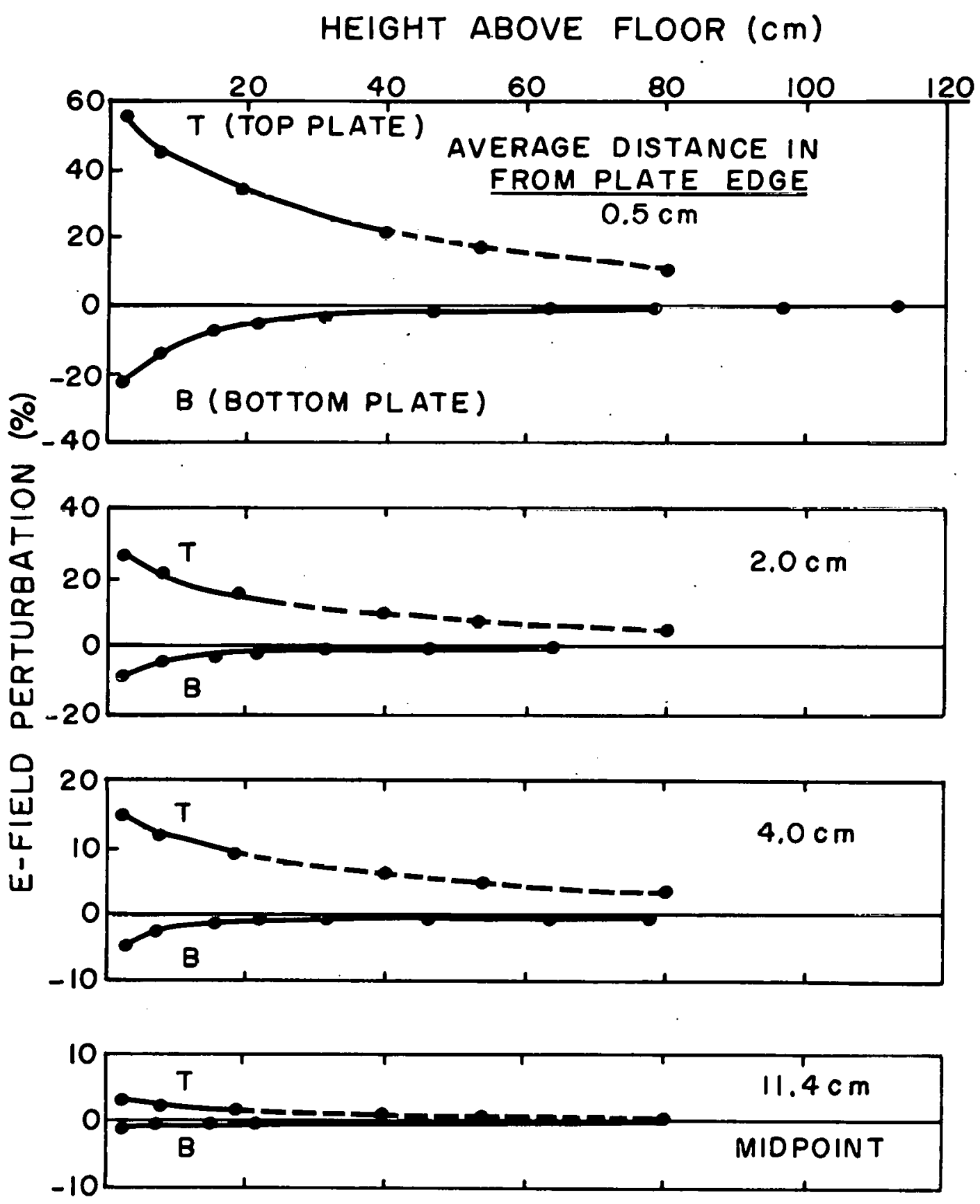

Figure B8. Perturbation of electric field (surface charge density) at plate surfaces because of floor proximity. 
WALL DISTANCE TO PARALLEL PLATE EDGE $(\mathrm{cm})$
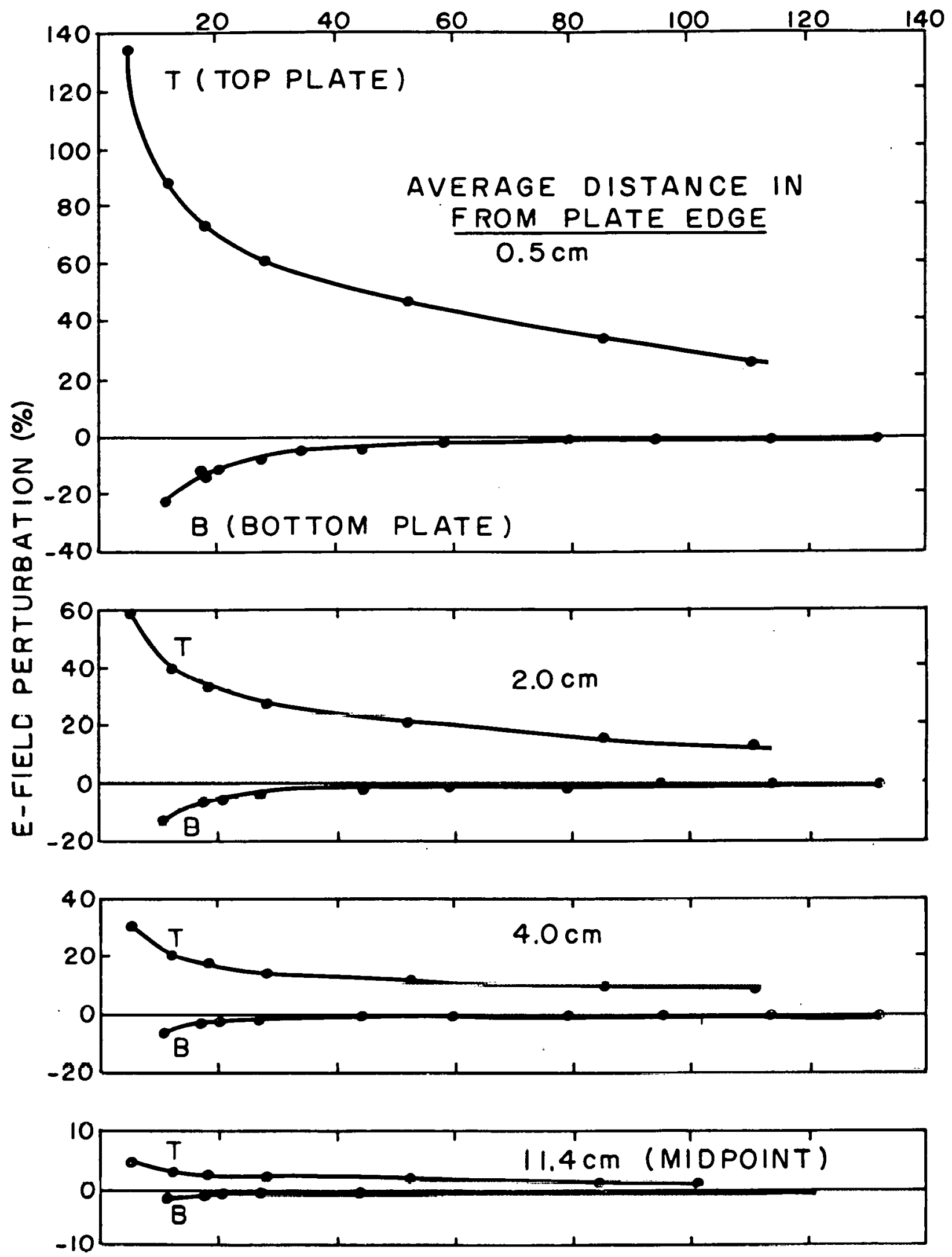

Figure B9. Perturbation of electric field (surface charge density) at plate surfaces because of wall proximity. 
Figure $B 8$ shows how the E-field along the bottom plate (B) is disturbed as a function of height above the floor. The distance between the plate edge and nearest wall is about $150 \mathrm{~cm}$ for all heights.

Figure $\mathrm{B} 9$ shows similar results as the distance between the parallel plate edge and wall is varied. It is noted that for the outermost element $(0.5 \mathrm{~cm})$, the field is normalized to zero perturbation at $170 \mathrm{~cm}$. Field perturbations due to the wall actually continued to greater distances for this element, but the limited room size prevented gathering of additional data (the influence of the opposite wall became apparent). The height above the floor was fixed near $150 \mathrm{~cm}$.

Because the E-field at the surface of the energized plate could not be directly monitored (with current measuring electronics), the perturbations at the energized plate were determined indirectly by raising the potential of the ground planes to the value of the energized plate and measuring the perturbation of the grounded plate as shown schematically in Figure B10.

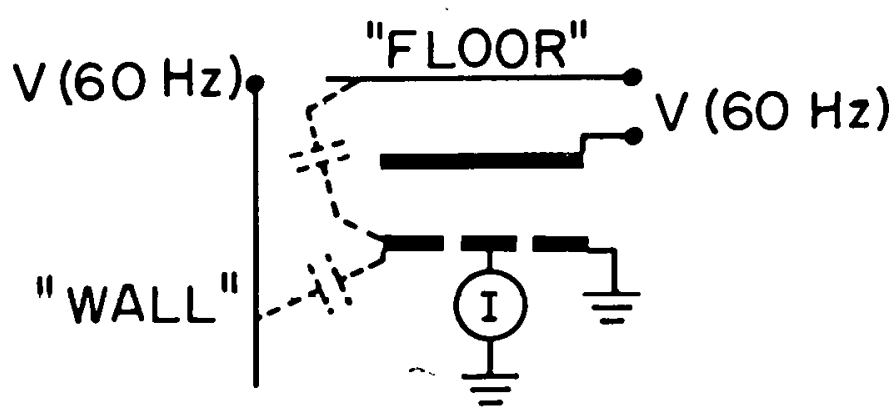

Figure B10

With this configuration, the distance between an energized "wal1" $2.5 \mathrm{~m} \times 2.3 \mathrm{~m}$ and a "floor" $1 \mathrm{~m} \times 1 \mathrm{~m}$ was varied and the perturbation of the E-field at the grounded plate was measured. The results $(T)$ are shown in Figures $B 8$ and $B 9$. The data show that under normal conditions (i.e., wails and floor at zero 
potential) a much greater perturbation occurs at the energized plate. Because the "floor" in Figure B10 was represented by a $1 \mathrm{~m} x^{\prime} 1 \mathrm{~m}$ screen, the accuracy of the floor perturbation data in Figure B8 is not well known. The accuracy improved as the distance was decreased.

(iii) Effectiveness of Guard Rings For Reducing External Perturbations

Guard rings have commonly been used to isolate the E-field between the plates from external perturbations as well as to establish a larger region of field uniformity by reducing the effect of fringing fields. Theoretical treatments of the problem for the case of cylindrical symmetry are described below.

From visits and discussions with workers concerned with field meter calibrations, it has been learned that the number of guard rings employed has varied from three or four to much larger numbers for plate spacings of comparable size. This prompted a systematic study of the effectiveness of guard rings using the smaller parallel plate structure.

Initially, thirty-one equally spaced guard rings (0.3 $\mathrm{mm}$ diameter copper wire) were used to grade the electric field at the perimeter of the parallel plates as shown in Figure $\mathrm{B} 11$. The potentials applled to the guard rings were yraded with a resistor divider linearly from top to bottom within a few tenths of a percent. (The resistor values chosen were all within $\pm 0.1 \%$ of $6.203 \times 10^{3}$ ohms, a value which is sufficiently small to shunt stray capacitance). The distance between the floor and the parallel plate structure was changed and the electric field was monitored at selected points along the bottom plate. Similarly, the distance between the wall and the parallel plate structure was varied and the E-field distribution was again monitored. This procedure was repeated with the number of guard rings successively reduced to $15,7,3$, and 1 .

Figure $\mathrm{B} 12$ shows how the E-field along the bottom plate is perturbed as a function of height above the floor and for different numbers of guard rings. 
Similar data are shown in Figure B13 as the distance between a nearby wall and the

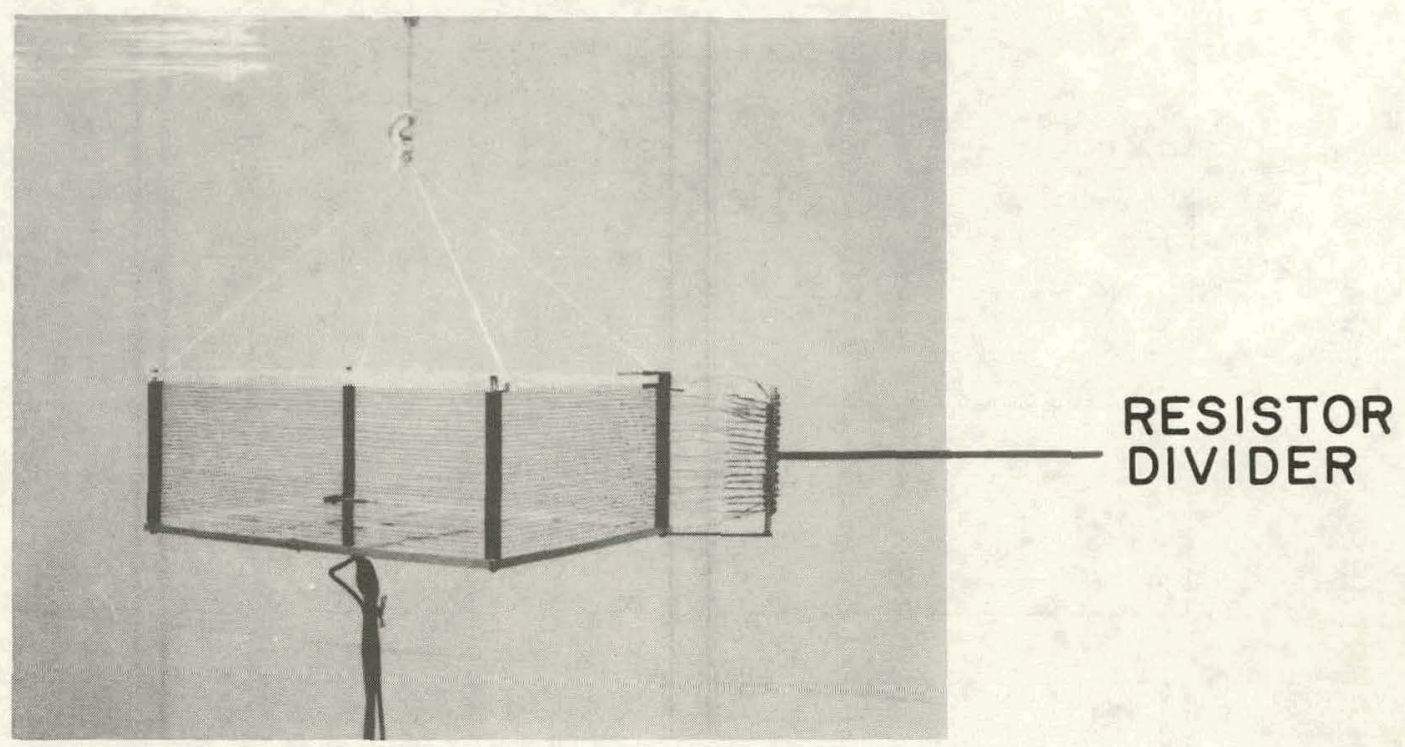

Figure B11. Parallel plates with 31 guard rings.

parallel plate edge is varied. Although error bars are not indicated, the perturbation values are estimated to be accurate within $\pm 0.5 \%$.

Discussion: The data in Figures $B 8$ and $B 9$ indicate that a parallel plate system with one plate grounded is very susceptible to perturbations from ground planes, even when they are quite distant. While the results show the perturbed field only on the inner plate surfaces, the qualitative conclusion can be made that the E-field will be enhanced at and near the energized plate (T) and will decrease as the grounded plate $(B)$ is approached. Even at the center of the plates, a measurable change can be expected when the walls are within a distance of two plate spacings away. This was confirmed experimentally with the $3 \mathrm{~m} \times 3 \mathrm{~m} \times 1 \mathrm{~m}$ structure described in Section II.C. 3 using the commercial field meters to measure $E$. 


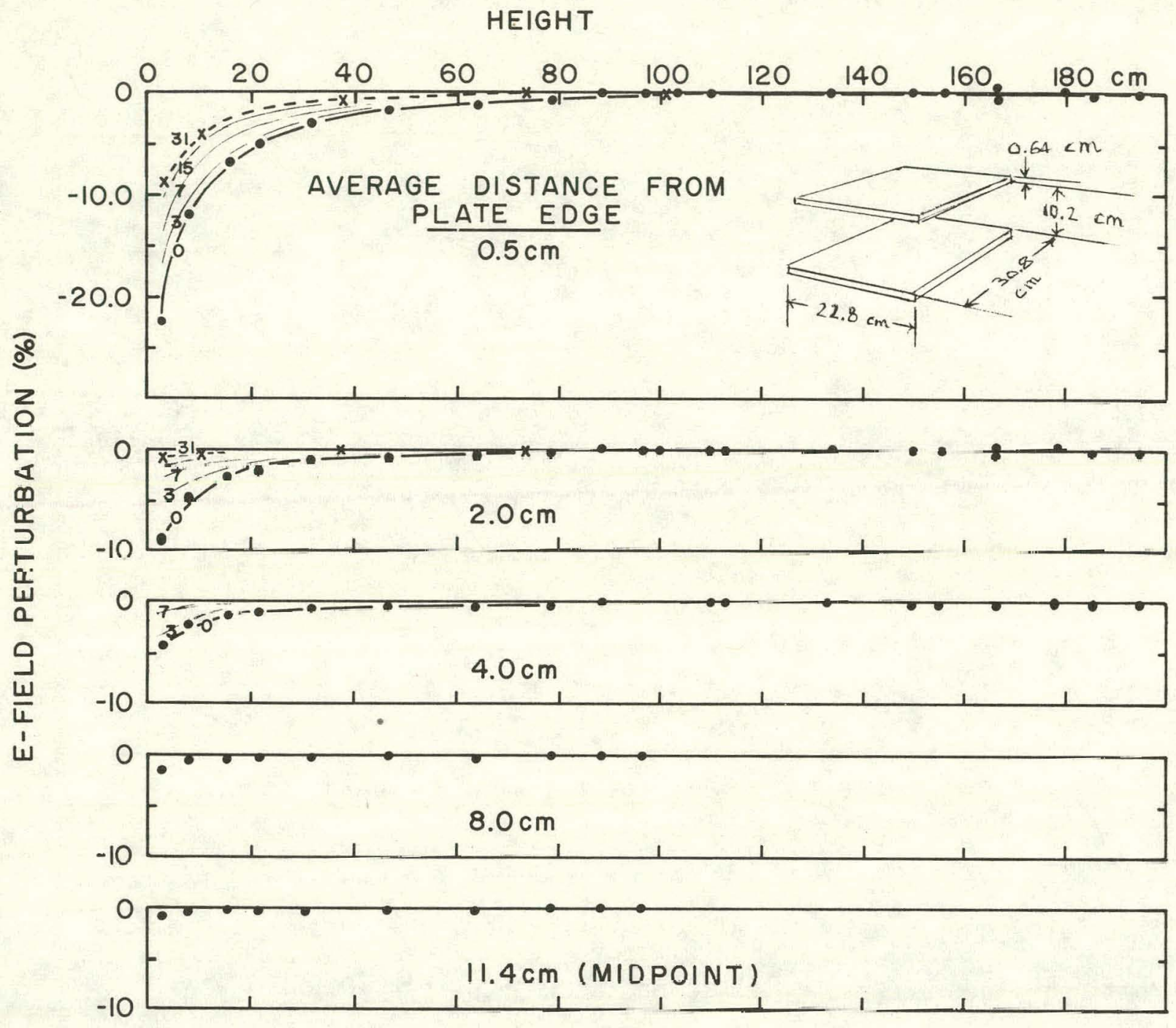

rigure B12. E-field perturbation as a function of height above the floor and number of guard rings $(31,15,7,3,0)$.

The data in Figures $\mathrm{B} 12$ and $\mathrm{B} 13$ show that a few guard rings are relatively inefficient for isolating and creating a greater region of uniform field between the plates with one plate grounded. They do improve conditions, however, and considerable advantage was gained in their use with one of the smaller calibration facilities described in Section II.C.4. 


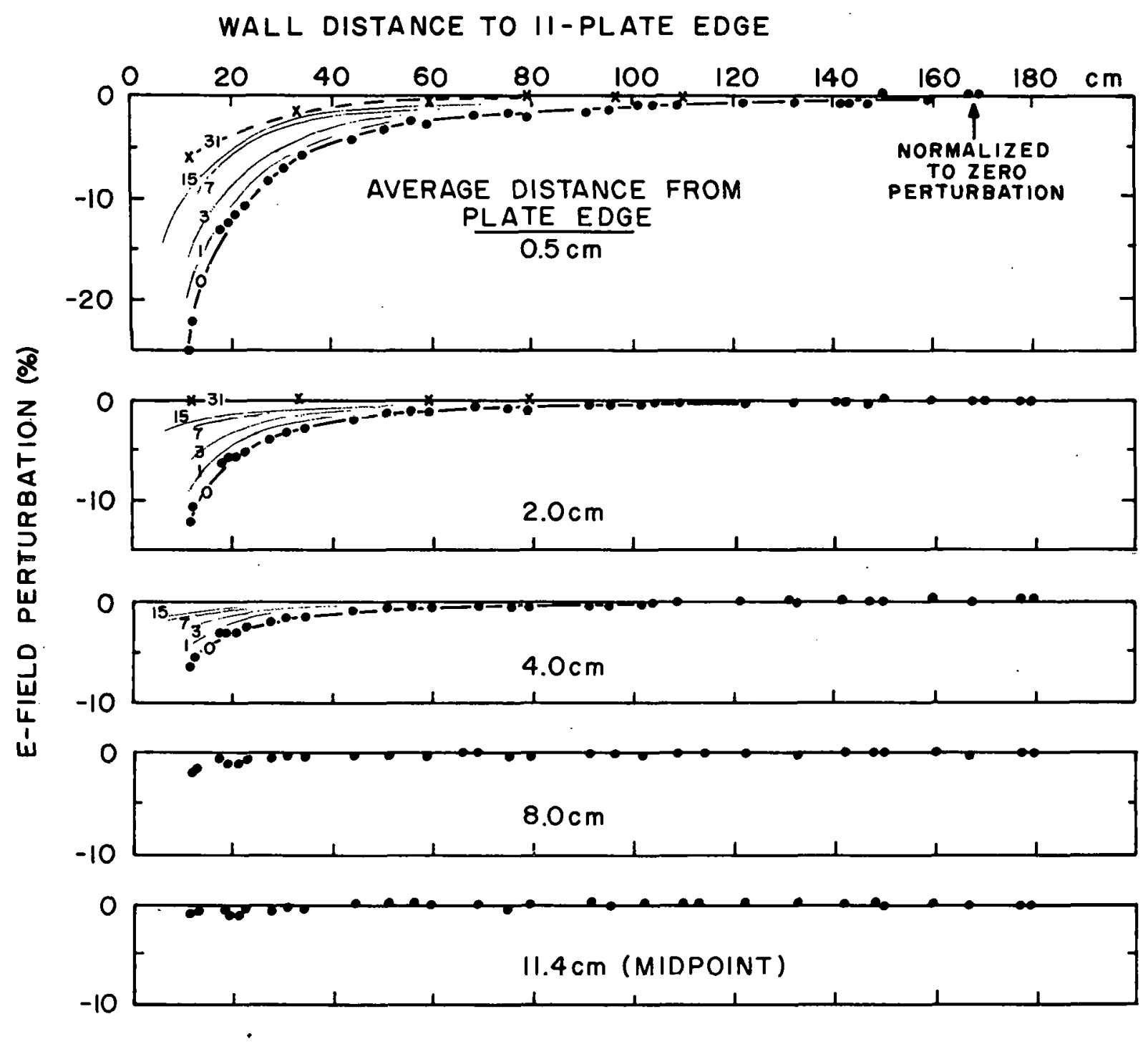

Figure B13. E-field perturbation as a function of distance from wall and number of guard rings $(31,15,7,3,1,0)$.

(iv) Parallel Plates With Guard Rings

The region of near-uniform field between two finite parallel plates may be increased by the use of guard rings to properly grade the potential in the space between the outside edges of the plates. A detailed discussion of this technique is presented in the article, "An Absolute Electrometer for the Measurement of 
High Alternating Voltages," by H. B. Brooks, et. al [18]. Because the theoretical development is relatively straightforward, only an outline is presented here. Further details as well as a description of a parallel plate apparatus employing guard rings can be found in Ref. 18.

The geometry of the problem is shown in Figure B14. Two circular plates of radius $R$ are separated by a distance $b$. A voltage divider, made up of capacitors of value $C$ provides for a nearly linear potential change of the guard rings along the bounding cylindrical surface. A limit on the degree of linearity of the change in guard ring potential is set principally by capacitive coupling to surrounding objects.

The problem is attacked by first determining the electric potential, $V(r, z)$, in the cylindrical volume by solving Laplace's equation

$$
\frac{\partial^{2} V}{\partial r^{2}}+\frac{1}{r} \frac{\partial V}{\partial r}+\frac{\partial^{2} V}{\partial z^{2}}+\frac{1}{r^{2}} \frac{\partial^{2} V}{\partial \phi^{2}}=0
$$

The electric field is then found by calculating the gradient of $V(r, z)$.

Because of the symmetry with respect to $\phi$, the last term in Equation $B 5$ vanishes. The boundary conditions for $V(r, z)$ are

$$
\begin{aligned}
& z=0, v=0 \text { for all } r \text { values } \\
& z=b, v=v_{a} \text { for all } r \text { values } \\
& r=R, v=v_{R}(>)
\end{aligned}
$$

where $V_{R}(z)$ describes the potential distribution at the cylindrical boundary. It is assumed to have the form

$$
V_{R}(z)=V_{a} \cdot \frac{z}{b}+V_{a} \sum_{k=1}^{\infty} G_{k} \sin (k \pi z / b)
$$




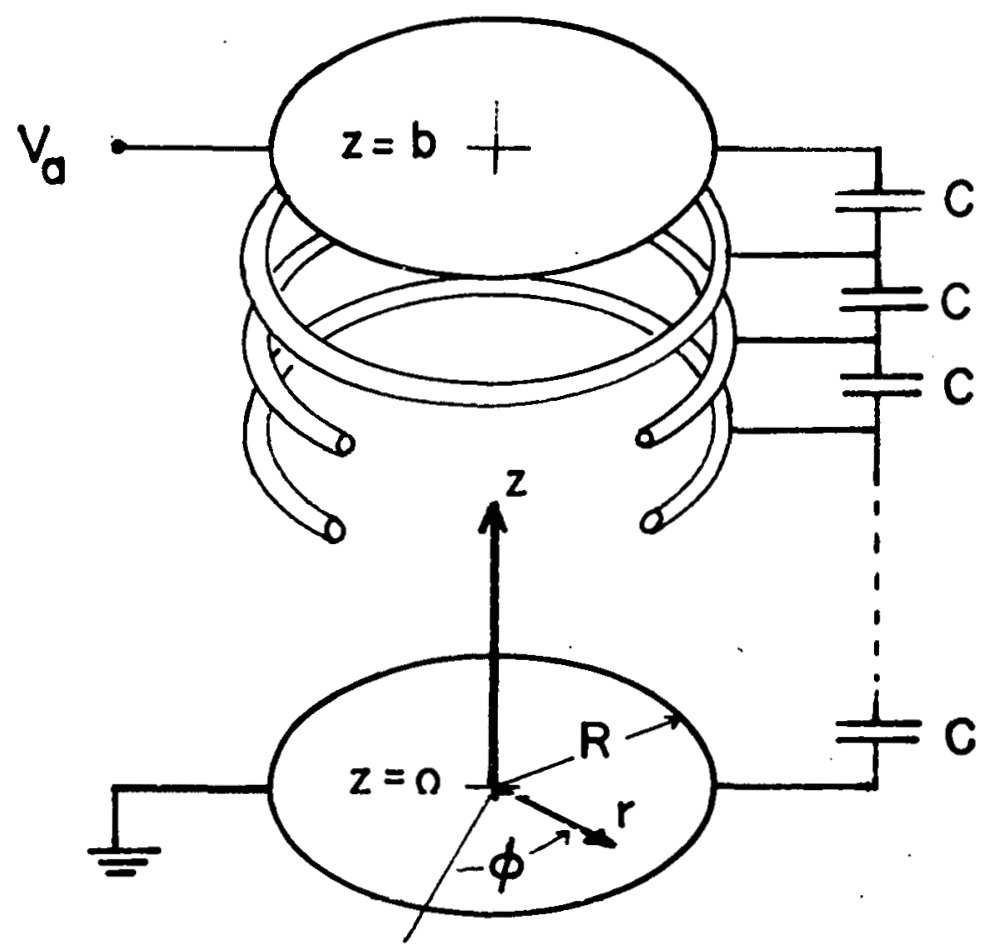

Figure $B 14$

where the first term describes the ideal linear change in potential and the second term is a Fourier sine series giving the departures from linearity.

The procedure for solving Laplace's equation is the well known separation of variables method [19]. A solution of the form

$$
V=V_{a} z / b+F_{1}(z) F_{2}(r)
$$

is assumed. Carrying out the differentiation indicated by Equation Bb, leads to two differential equations. One is Bessel's equation of zeroeth order

$$
\frac{\partial^{2} F_{2}}{\partial r^{2}}+\frac{1}{r} \frac{\partial F_{2}}{\partial r}-\mu^{2} F_{2}=0
$$


with the solution

$$
F_{2}=J_{0}(i \mu r)
$$

The second equation

$$
\frac{\partial F_{1}}{\partial z^{2}}+\mu^{2} F_{1}=0
$$

with the solution

$$
F_{1}(z)=V_{a}(A \cos \mu z+B \sin \mu z)
$$

Because the separation constant $\mu$ is arbitrary, the general solution to Laplace's equation is found by summing over all possible solutions (i.e., with all possible $\mu$ 's) and hence

$$
v=v_{a} z / b+v_{a} \sum_{k=1}^{\infty}\left[A_{k} \cos \mu_{k} z+B_{k} \sin \mu_{k} z\right] J_{0}\left(i \mu_{k} r\right)
$$

Applying the boundary conditions, B6 and B7 yields

$$
V=v_{a} z / b+v_{a} \sum_{k=1}^{\infty} G_{k} \sin (k \pi z / b) \cdot \frac{J_{0}(i k \pi r / b)}{J_{0}(i k \pi R / b)}
$$

The Bessel functions $J_{0}$ with imaginary arguments can be replaced by modified Bessel functions [20] $I_{0}$ with real arguments. We have then 


$$
V=V_{a} z / b+V_{a} \sum_{k=1}^{\infty} G_{k} \sin (k \pi z / b) \cdot \frac{I_{0}(k \pi r / b)}{I_{0}(k \pi R / b)} .
$$

The Fourier coefficients, $G_{k}$, are given by the approximation [18]

$$
G_{k}=\frac{2}{n+1} \sum_{m=1}^{n}\left(\frac{V_{m}}{V_{a}}-\frac{m}{n+1}\right) \sin \left(\frac{k \pi m}{n+1}\right) \quad k=1,2 \ldots n .
$$

Here $n$ is the number of guard rings and $V_{m}$ is the measured potential of the mth guard ring. As $\mathrm{n}$ is increased, the accuracy of the approximation, Equation B16, improves, but the interested reader is encouraged to consult the relevant portions of Ref. [18] to understand some of the subtleties of this approximation. With increasing numbers of guard rings, $n$, the values of $G_{k}$ decrease so that all values of $G_{k}$ need not be used in the summation, Equation B15.

The two components of electric field are given by

$$
\begin{aligned}
& E_{z}=-\frac{\partial V}{\partial z} \\
& E_{r}=-\frac{\partial V}{\partial r} .
\end{aligned}
$$

Performing the differentiation on Equation 15, we have

$$
E_{z}=-E_{0}\left[1+\sum_{k=1}^{\infty} k \pi G_{k} \cos (k \pi z / b)\right] \cdot \frac{I_{0}(k \pi r / b)}{I_{0}(k \pi R / b)}=E_{0} M
$$

and 


$$
E_{r}=-E_{0}\left[\sum_{k=1}^{\infty} k \pi G_{k} \sin \frac{k \pi z}{b} \frac{I_{1}(k \pi r / b)}{I_{0}(k \pi R / b)}\right]=E_{0} N
$$

where $E_{0}=V_{a} / b$. The magnitude of the electric field relative to the uniform value in the interior, if the plates were sufficiently large, is given by

$$
\frac{E}{E_{0}}=\left[M^{2}+N^{2}\right]^{1 / 2} .
$$

An alternative approach to the use of guard rings is to replace the rings with bands of metal separated by narrow air gaps [13]. Far greater isolation from nearby ground planes is attainable. 


\section{Ground Referenced Electric Field Meters}

As noted earlier, ground referenced E-field meters have been used to measure transmission line fields. Perhaps the most well known are the Miller meter. [1] and the "one square meter panel" [2] which has been used for calibration purposes. The principle of operation of these two instruments is the same and an analys is is now presented which indicates under what conditions the devices yield correct resulls.

Schematic views of the Miller meter and the "one square meter panel" in the presence of a quasistatic-uniform transmission line E-field, $E_{0}$ sin $\omega t$, are shown in Figure $\mathrm{Cl}$.

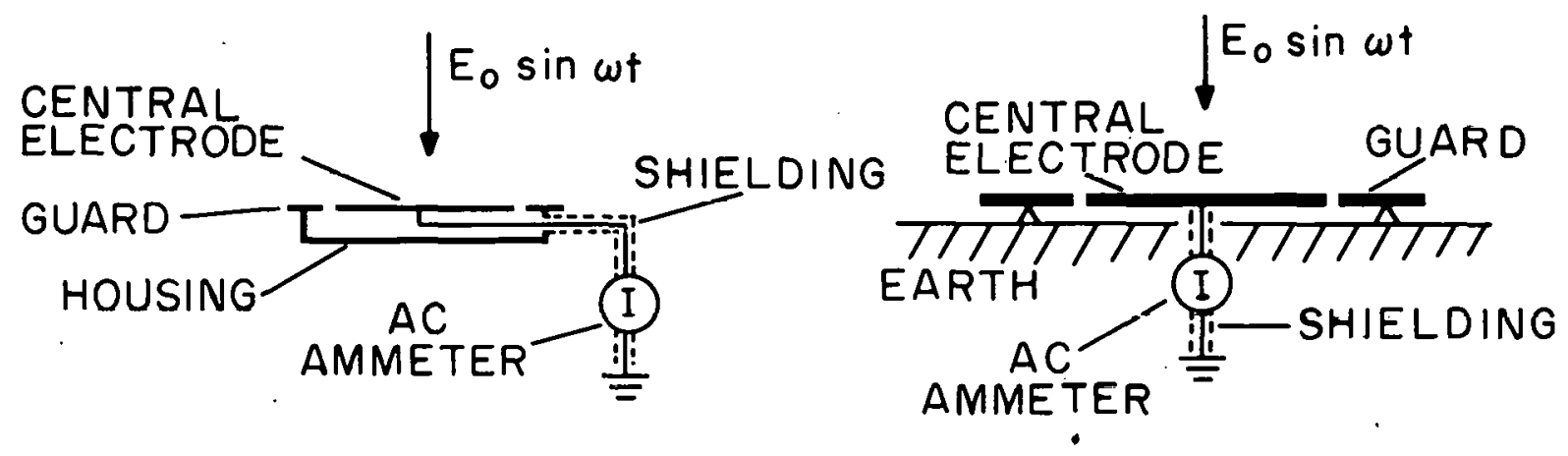

Figure $\mathrm{Cl}$

Application of Gauss' Law to the central electrodes permits the derivation of the relation

$$
I=\omega \varepsilon_{0} A E \sin \omega t
$$


where I is the induced current, $\varepsilon_{0}$ is the electric permittivity of space, $E$ is the electric field at the metal surface and $A$ is the area of the central electrode. Measurement of the current permits the determination of the E-field at the metal surface. While Equation $\mathrm{Cl}$ is a valid expression for determining $\mathrm{E}$ at the surface of the metal, it can be used to determine the uniform field, $E_{0}$, only under certain conditions as is shown below.

For purposes of analysis, both devices are roughly approximated by the segmented conducting sphere sketched in Figure C2. It can be shown that if a conducting sphere of radius a, having a potential $V$, is introduced into a quasistatic uniform

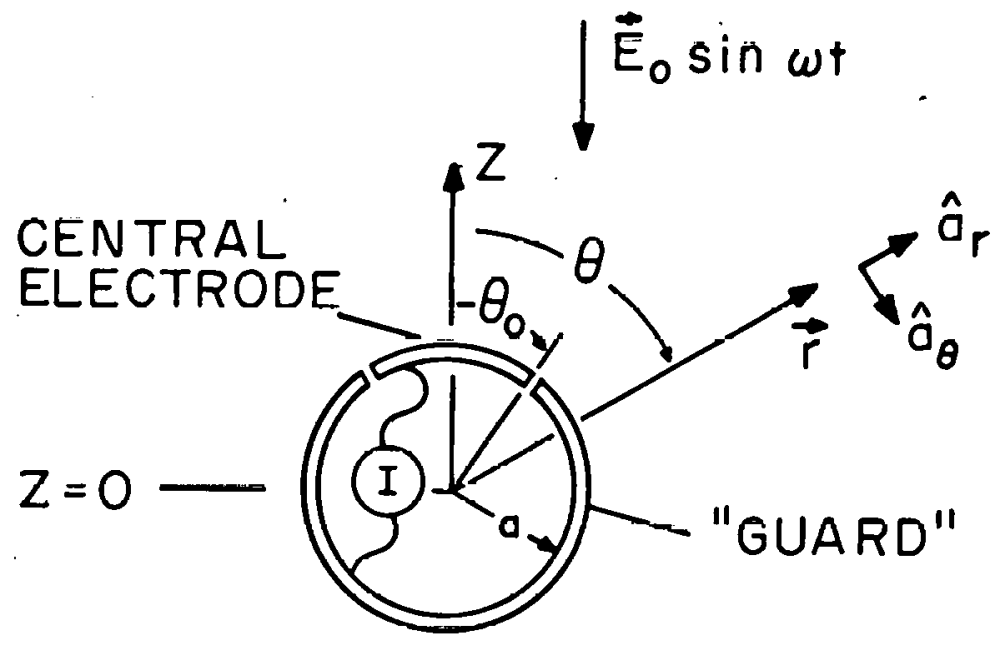

Figure C2

E-field, $E_{0}$ sin $\omega t$, the perturbed electric field is given by [16]

$$
\begin{aligned}
\vec{E}=-\hat{a}_{r} & {\left[\frac{(K-V) a}{r^{2}}+E_{0} \sin \omega t\left[1+2\left(\frac{a}{r}\right)^{3}\right] \cos \theta\right] } \\
& +\hat{a}_{\theta} E_{0} \sin \omega t\left[1-\left(\frac{a}{r}\right)^{3}\right] \sin \theta
\end{aligned}
$$


where $K$ is the space potential at $z=0$ prior to the introduction of the sphere. The explicit sinusoidal time dependence of $K$ is suppressed. At the surface of the sphere $(r=a)$, the magnitude of $E$ is

$$
E=\frac{K-V}{a}+3 E_{0} \sin \omega t \cos \theta
$$

and the surface charge density is given by

$$
\sigma=\varepsilon_{0} E=\varepsilon_{0}\left[\frac{(K-V)}{a}+3 E_{0} \sin \omega t \cos \theta\right] .
$$

The total induced charge $Q$ on the central electrode is found from the surface integral

$$
\begin{aligned}
Q & =\int_{0}^{\theta_{0}} \int_{0}^{2 \pi} \sigma a^{2} \sin \theta d \phi d \theta \\
& =C_{1}(K-V)+C_{2} E_{0} \sin \omega t .
\end{aligned}
$$

Here $c_{1}$ and $c_{2}$ are constants. The induced current for two cases is now considered. (a) $K=V$. If the potential of the sphere is the same as the space potential $K$,

$$
I=\frac{d Q}{d t}=\omega C_{2} E_{0} \cos \omega t
$$

and the uniform field may be determined by measuring the current. Examples of this case are the Miller meter and the one square meter panel operating at ground level, i.e., $V=K=0$. Allowing the sphere to electrically "float" in space is yet another example where $K=V$ and Equation $C 6$ is valid. The commercial ac E-field meters currently available in the U.S. operate under this condition. 
(b) $K \neq V$. If the sphere's potential is maintained at some constant value, the induced current becomes

$$
I=\frac{d Q}{d t}=\omega C_{1} K+C_{2} \omega E_{0} \sin \omega t
$$

Thus, the induced current will depend on the space potential $K$ as well as the uniform field magnitude. Moving the sphere to higher elevations above ground, where the space potential is larger, will result in greater induced current. For example, choosing $V=0$ and $K \neq 0$ corresponds to the case of the Miller meter or the "one square meter panel" being raised above the ground level (with the ammeter still connected to true ground). This condition can develop for both devices even when they are resting on the earth's surface. If the ground is sufficiently dry, the true ground could be well below the surface and the induced current would then be proportional to something other than just the uniform field value $E_{0}$. 


\section{Appendix D}

\section{Miniature Electric Field Probe}

The theoretical discussion in Appendix $C$ notes that a spherical E-field probe, if it is to work properly, must be at the same electric potential as the unperturbed space potential at the location of the sphere's center prior to introduction of the probe. This condition is achieved for large E-field meters by electrically isolating or "floating" the probe (sensing plates) from ground with an insulating rod which is used to support the meter in the fleld; the battery operated electronics are incorporated between the sensing plates.

A miniature E-field probe which is well removed from its battery operated electronics has been developed and is shown schematically in Figure Dl. Shielded wires rather than glass fiber optics $[6,21]$ are used to couple the probe to the electronics. The induced charge on the hemispherical sensing plates is measured (similar to Type A meter) with a differential amplifier that is contained in an electrically biased chassis box. This bias is adjusted to be the space potential in the equatorial plane close to the equator of the spherical probe and is set using the potentiometer--space potential probe--high impedance voltmeter combination. A voltage null indicates when the bias is appropriately set. The space potential probe consists of the open end of an otherwise $100 \%$ shielded miniature coaxial cable and is brought near the field probe in the equatorial plane (see Figure D1 inset). Because one input of the differential amplifier is effectively connected to the chassis, the potential of both hemispherical sensing plates and the shielding will be "floating" at the appropriate potential when a null is achieved [22]. The space potential probe is removed during field measurements. 
Calibration of the E-field probe and electronics is readily performed with a small parallel plate structure. Because of its size $(1.2 \mathrm{~cm}$ diameter $)$, the probe will not significantly perturb the parallel plate surface charge densities (Appendix B.i) when the plate spacing is as small as $20 \mathrm{~cm}$.

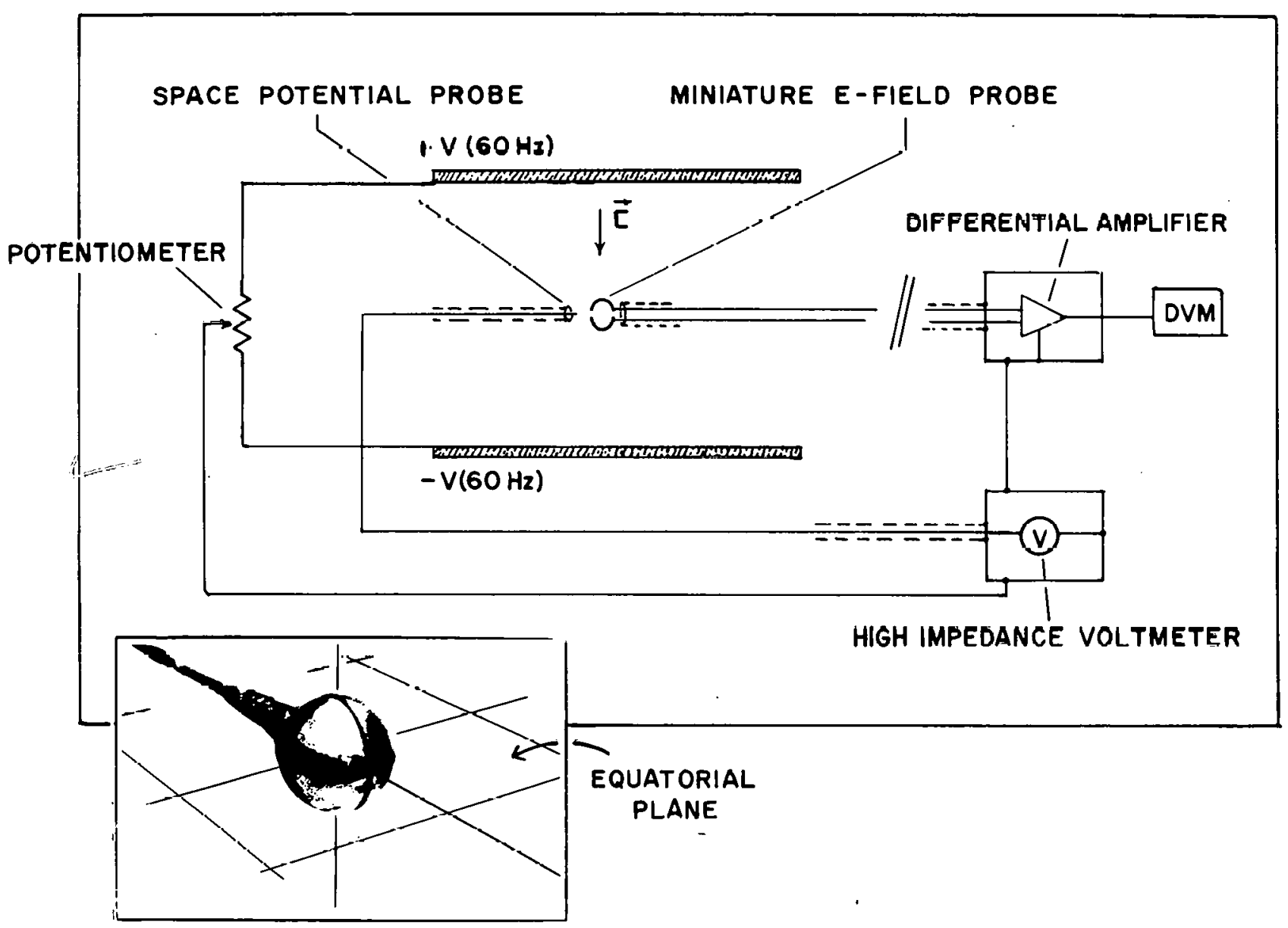

Figure D1. Simplified schematic view of miniature E-field probe, space potential probe, and associated electronics. The inset shows the E-field probe sensing surfaces. Two orthogonal field components can be measured without changing the probe orientation. 
The miniature E-field probe has proven to be useful in obtaining E-field "maps" for parallel plate structures used for calibrating E-field meters (Section II.B.4). It has also been used in a similar fashion to characterize E-fields in small parallel plate structures used for biological experiments.

Probes consisting of miniature parallel plates have also been fabricated and used with success in the above measurements. The spherical probe shown in the inset of Figure DI, with sensing elements consisting of quarter spheres, is designed to measure two perpendicular components of the E-field.

\section{Acknowledgment}

The authors are pleased to acknowledge the careful editorial and typographical assistance of J. C. Palla during the preparation of the manuscript. 


\section{Notes and References}

1. C. J. Miller, "The Measurements of Electric Fields in Live Line Working," IEEE Trans. Power App. Syst., vol. PAS-86, pp. 493-498, April 1967.

2. Transmission Line Reference Book - $345 \mathrm{kV}$ and Above, Electric Power Research Institute, Palo Alto, California, 1975, p. 274.

3. The surface charge density is given by $3 \varepsilon_{0} E \cos \theta[J . R$. Reitz and F. J. Milford, Foundations of Electromagnetic Theory. (Addison-Wesley, Reading, Mass., 1960) p. 52]. Integration over the hemisphere gives Eq. 2.

4. The percent error is calculated by first determining the analog reading when . a given harmonic is present (see, e.g., E. Frank, Electrical Measurement Analysis, McGraw-Hill, 1959, pp. 303-309) and comparing it with the true rms value for the same waveform. That is

$$
\% \text { Error }=\left[\frac{\text { "Averaged RMS" Reading - True RMS Reading }}{\text { True RMS Reading }}\right] \times 100 .
$$

See also

M. R. Negrete, "The Effects of Distortion on AC Voltmeters," Wescon Technical Papers, Vol. 9, Part 6 (1965) and

W. G. Smith, "...What's Wrong With DVM AC Measurements," EEE: The Magazine of Circuit Design Engineering, March 1971, pp. 52-56.

5. Private communication from T. D. Bracken, Bonneville Power Administration, Portland, Uregon.

6. L. Wilhelmy, "Potentialfreie Feldstärkemessung im raumladungsfreien periodisch zeitabhängigen und transienten elektrischen Feld," Thesis, University of Stuttgart, 1972. (An English translation is available from Xerox University Microfilms, Ann Arbor, Michigan.

7. See IEEE Power Engineering Society Special Publication Number 10 (1975) for translation of Russian article "Rules and Regulations on Labor Protection at. 400 , 500, and $750 \mathrm{kV} \mathrm{AC} \mathrm{Substations} \mathrm{and} \mathrm{Overhead} \mathrm{Lines} \mathrm{of} \mathrm{Industrial}$ Frequency (In the U.S.S.R.)."

8. V. P. Korobkova, Yu. A. Morozov, M. D. Stolarov, and Yu. A. Yakub, "Influence of the Electric Field in 500 and $750 \mathrm{kV}$ Switchyards on Maintenance Staff and Means For Its Protection," 1972 CIGRE Paper 23.06.

9. Yu. A. Morozov and 0. M. Gromov, "Device For The Measurement of Industrial Frequency Electric Field Intensity," Ref. 7 Translation.

10. L. B. Young, Report to the U.S. Environmental Protection Agency on Effects of Extremely High Voltage Transmission, July 1, 1975. 
11. P. D. Thacher, "Fringing Fieids in Kerr Cells," Sandia Report SLA-74-0302 (1974). See also IEEE Trans. Elect. Insul., vol. EI-11, pp. 40-50, June 1976.

12. A clear introduction is provided in S. Ramo and J. R. Whinnery, Fields and Waves in Modern Radio (John Wiley \& Sons, 1953), pp. 127ff. See also Ref. 14.

13. B. Barber, "The Basic Design Data of a Guarded-Field Thimble Ionization Chamber: A Theoretical Investigation," Phys. Med. and Biol., vol. 4, 1-9, 1959; L.A.W. Kemp and B. Barber, "The Basic Design Data of a Guarded-Field Thimble Ionization Chamber: A Precision Ionometric Investigation," Phys. Med. and Biol., vol. 3, 123-137, 1958.

14. See E. Weber, Electromagnetic Theory. (Dover, New York, 1965), p. 131ff for outline of derivation.

15. See Ref. 3, p. $59 \mathrm{ff}$.

16. P. Moon and D. E. Spencer, Field Theory For Engineers (D. Van Nostrand, 1961), p. $225 \mathrm{ff}$.

17. See Ref. 3, pp. 25-26.

18. H. B. Brooks, F. D. Defandorf, and F. B. Silsbee, J. Research NBS 20, 253 (1938). See also Ref. 12, p. 156 for details of a related problem.

19. R. V. Churchill, "Fourier Series and Boundary Value Problems," (McGraw-Hill, 1941), p. $24 \mathrm{ff}$.

20. M. Abramowitz and I. A. Stegun, ed. Handbook of Mathematical Functions, NBS Applied Mathematics Series 55 (1964), p. 374ff.

21. A. R. Valentino, Proc. IEEE International Symposium on Electromagnetic Compatibility, p. 265 (July 1972) (unpublished); available as IITRI Report No. AD 742128 from NTIS, Springfield, Virginia 22161.

22. In a uniform E-field, the unperturbed space potential, $\phi_{0}$, at $Z=0$ (Figure C2) will be equal to the potential value in the equatorial plane after an electrically isolated sphere is introduced into the field. In the present case, the sphere is initially at some arbitrary potential $\phi_{\neq} \neq \phi_{0}$, and the equatorial plane near the sphere will not be at the appropriate potential $\phi_{0}$. As the voltage difference between the sphere and space potential probe is reduced, however, the potential surfaces near the sphere approach the geometry which is characteristic for an electrically isolated sphere. 